\title{
Transmit Antenna Selection for Multiple-Input Multiple-Output Spatial Modulation Systems
}

\author{
Ping Yang, Senior Member, IEEE, Yue Xiao, Member, IEEE, Yong Liang Guan, Member, IEEE, \\ Shaoqian Li, Fellow, IEEE, and Lajos Hanzo, Fellow, IEEE
}

\begin{abstract}
The benefits of transmit antenna selection (TAS) invoked for spatial modulation (SM) aided multiple-input multiple-output (MIMO) systems are investigated. Specifically, we commence with a brief review of the existing TAS algorithms and focus on the recently proposed Euclidean distance-based TAS (ED-TAS) schemes due to their high diversity gain. Then, a pair of novel ED-TAS algorithms, termed as the improved QR decomposition (QRD)-based TAS (QRD-TAS) and the error-vector magnitude-based TAS (EVM-TAS) are proposed, which exhibit an attractive system performance at low complexity. Moreover, the proposed ED-TAS algorithms are amalgamated with the low-complexity yet efficient power allocation (PA) technique, termed as TAS-PA, for the sake of further improving the system's performance. Our simulation results show that the proposed TAS-PA algorithms achieve signal-to-noise ratio (SNR) gains of up to $9 \mathrm{~dB}$ over the conventional TAS algorithms and up to $6 \mathrm{~dB}$ over the TAS-PA algorithm designed for spatial multiplexing systems.
\end{abstract}

Index Terms-Antenna selection, MIMO, power allocation, spatial modulation, link adaptation.

\section{INTRODUCTION}

$\mathbf{S}$ PATIAL modulation (SM) and its variants constitute a class of promising low-complexity and low-cost multipleinput multiple-output (MIMO) transmission techniques [1]-[5]. However, the conventional SM schemes only achieve receiverdiversity, but no transmit diversity [6]. To circumvent this impediment, recently some SM solutions have been proposed [7]-[11] on how to glean a beneficial transmit-diversity gain both with the aid of open-loop as well as closed-loop transmitsymbol design techniques.

As an attractive closed-loop regime, transmit antenna selection (TAS) constitutes a promising technique of providing a

Manuscript received October 10, 2015; revised February 22, 2016; accepted March 24, 2016. This work was supported of the National Science Foundation of China under Grant 61501095, in part by the National High-Tech R\&D Program of China ("863" Project under Grant 2014AA01A707), and in part by the European Research Council's Advanced Fellow Grant. The associate editor coordinating the review of this paper and approving it for publication was V. Raghavan.

P. Yang, Y. Xiao, and S. Li are with the National Key Laboratory of Science and Technology on Communications, University of Electronic Science and Technology of China, Sichuan 611731, China (e-mail: yplxw@163.com; xiaoyue@uestc.edu.cn; 1sq@uestc.edu.cn).

Y. L. Guan is with the School of Electrical and Electronic Engineering, Nanyang Technological University, Singapore (e-mail: eylguan@ntu.edu.sg).

L. Hanzo is with the School of Electronics and Computer Science, University of Southampton, Southampton SO17 1BJ, U.K. (e-mail: 1h@ecs.soton.ac.uk).

Color versions of one or more of the figures in this paper are available online at http://ieeexplore.iee.org.

Digital Object Identifier 10.1109/TCOMM.2016.2547900 high diversity potential as offered by the classic MIMO architectures. TAS has been lavishly researched in the context of spatial multiplexing systems [12]. As a new MIMO technique, SM can also be beneficially combined with TAS. Recently, several TAS algorithms have been conceived for the class of SM-MIMO systems with the goal of enhancing either its bit error rate (BER) or its capacity [13]-[20]. In [13], a normbased TAS algorithm was proposed for providing diversity gain. In [14], a closed-form expression of the SM scheme's outage probability was derived for norm-based TAS. In [16], a twostage TAS-based SM scheme was proposed for overcoming the specific constraint of SM, namely that the number of transmit antennas has to be a power of two. In [17], a novel TAS criterion was proposed for circumventing the detrimental effects of antenna correlation. In [18], the joint design of TAS and constellation breakdown was investigated and a graph-based search algorithm was proposed for reducing the search complexity imposed. In [19], a low-complexity TAS algorithm based on circle packing was proposed for a transmitter-optimized spatial modulation (TOSM) system, which trades off the spatial constellation size against the amplitude and phase modulation (APM) constellation size for improving the system's average bit error probability (ABEP). The adaptive TAS algorithm conceived for TOSM was further developed in [20], where a low-complexity two-stage optimization was proposed for selecting the best transmission mode.

More recently, the research of TAS-aided SM has been focused on the optimization of the Euclidean Distance (ED) of the received constellation points, since they achieve a high diversity gain at a moderate complexity compared to other TAS criteria [21]-[24]. Specifically, in [21] and [22] the EDbased TAS algorithm (ED-TAS) was compared to the signalto-noise ratio (SNR)-optimized and capacity-optimized algorithms, and a low-complexity realization of ED-TAS, termed as the QR decomposition-based TAS (QRD-TAS) was proposed. The QRD-TAS algorithm constructs an ED-element matrix and exploits the QRD of the resultant matrix for reducing the imposed complexity. Moreover, in [24], the authors exploited the rotational symmetry of the APM adopted for the sake of reducing the complexity of QRD-TAS. Compared to directly optimizing the ED, in [23], Ntontin et al. proposed a low-complexity singular value decomposition-based TAS (SVD-TAS) algorithm for maximizing the lower bound of the ED. In [25], the complexity of SVD-TAS was reduced through an alternative computation of the singular value. In [26], the transmit diversity order of ED-TAS was quantified. In [27], the authors proposed several low-complexity TAS schemes relying 
on exploiting the channel's amplitude, the antenna correlation, the ED between transmit vectors and their combinations for selecting the optimal TA subset for the sake of improving the system's reliability. However, as shown in [21]-[27], the QRD-TAS achieves an attractive BER performance at the cost of adopting high-complexity QRD operations, while the low-complexity SVD-TAS may suffer some performance loss.

On the other hand, power allocation (PA) is another promising link adaptation technique for MIMO systems. Recently, PA has been extended to SM systems [28]-[31]. For example, in [28], an adaptive PA algorithm based on maximizing the minimum ED was proposed, which is capable of improving the system's BER performance, while retaining all the single-RF benefits of SM. Subsequently, this attractive PA algorithm was further simplified in [29]. However, to the best of our knowledge, the potential benefits of TAS intrinsically amalgamated with PA have not been investigated in SM-MIMO systems.

Against this background, the contributions of this paper are:

1) We investigate the benefits of ED-TAS and propose a pair of novel ED-TAS schemes for SM-MIMO systems. In these schemes, we first classify the legitimate EDs into three specific subsets and then invoke a carefully designed upper bound as well as a set-reduction method for the most dominant set imposing a high complexity.

2) Specifically, we propose an improved QRD-TAS, where a tighter QRD-based lower bound of the ED is derived to replace the SVD-based bound of [23]. A low-complexity method is proposed for directly calculating the bound parameters, in order to avoid the high-complexity QRD or SVD operations of [21]-[24]. More importantly, compared to the conventional SVD-TAS of [25], the achieved QRD-based tighter bound can achieve a better BER performance.

3) Moreover, for striking a flexible tradeoff in terms of the BER attained and the complexity imposed, we propose an error-vector magnitude based TAS (EVM-TAS), which exploits the error vector selection probability to shrink the search space. The relevant optimization metrics of EVM-TAS are also derived for different PSK and QAM schemes.

4) Finally, we intrinsically amalgamate the proposed EDTAS with the recently conceived PA technique of [29] for fully exploiting the MIMO channel's resources. A pair of different joint TAS-PA algorithms are conceived, which provide beneficial gains over both the conventional TAS algorithms and over the TAS-PA techniques designed for spatial multiplexing systems [32].

The organization of the paper is as follows. Section II introduces the system model of TAS-based SM, while Section III reviews the family of existing TAS algorithms designed for SM. In Section IV, we introduce the proposed QRD-TAS and EVM-TAS algorithms. In Section V, the joint design of the EDTAS and PA algorithms is proposed. Then, we carry out their complexity analysis. Our simulation results and performance comparisons are presented in Section VI. Finally, Section VII concludes the paper.

Notation: $(\cdot)^{*},(\cdot)^{T}$ and $(\cdot)^{H}$ denote conjugate, transpose, and Hermitian transpose, respectively. Furthermore, $\|\cdot\|_{F}$ stands for the Frobenius norm. $\mathbf{I}_{b}$ denotes a $(b \times b)$-element identity matrix and the operator $\operatorname{diag}\{\cdot\}$ is the diagonal operator. $\mathcal{R}\{\mathbf{x}\}$ and $\mathcal{J}\{\mathbf{x}\}$ represent the real and imaginary parts of $\mathbf{x}$ respectively.

142 143Q1 144

\section{SySTEM MODEL}

Consider a SM system having $N_{t}$ transmit and $N_{r} 147$ receive antennas, as depicted in Fig. 1. The frequency- 148 flat quasi-static fading MIMO channel is represented 149 by $\mathbf{H}=\left[\mathbf{h}(1), \mathbf{h}(2), \cdots, \mathbf{h}\left(N_{t}\right)\right] \sim \mathcal{C N}\left(0, \mathbf{I}_{N_{r} \times N_{t}}\right)$, where 150 $\mathbf{h}(1), \mathbf{h}(2), \cdots, \mathbf{h}\left(N_{t}\right)$ are the column vectors corresponding 151 to each transmit antenna (TA) in $\mathbf{H}$. The receiver first selects 152 $L$ TAs according to a specific selection criterion. Then, the 153 receiver sends this information to the transmitter via a feedback 154 link. As shown in [23], let $U_{u}$ denote the $u t h$ legitimate TA 155 subset, where we have

$$
\begin{aligned}
& U_{1}=\{1,2, \cdots, L\}, \\
& U_{2}=\{1,2, \cdots, L-1, L+1\}, \\
& \vdots \\
& U_{N_{U}}=\left\{N_{t}-L+1, \cdots, N_{t}\right\} .
\end{aligned}
$$

In Eq. (1), there are $N_{U}=\left(\begin{array}{c}N_{t} \\ L\end{array}\right)$ possible TA subsets, each of 157 which corresponds to an $\left(N_{r} \times L\right)$-element MIMO channel. As 158 shown in Fig. $1, \mathbf{b}=\left[b_{1}, \ldots, b_{L}\right]$ is the transmit bit vector in 159 each time slot, which contains $m=\log _{2}(L M)$ bits, where $M$ is 160 the size of the APM constellation. In SM, the input vector b is 161 partitioned into two sub-vectors of $\log _{2}(L)$ and $\log _{2}(M)$ bits, 162 denoted as $\mathbf{b}_{1}$ and $\mathbf{b}_{2}$, respectively. The bits in $\mathbf{b}_{1}$ are used for 163 selecting a unique TA index $q$ for activation, while the bits of 164 $\mathbf{b}_{2}$ are mapped to a Gray-coded APM symbol $s_{l}^{q} \in \mathbb{S}$. Then, the 165 SM symbol $\mathbf{x} \in \mathbb{C}^{L \times 1}$ is formulated as

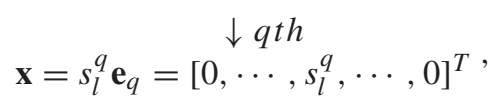

where $\mathbf{e}_{q}(1 \leq q \leq L)$ is selected from the $L$-dimensional basis 167 vectors (as exemplified by $\mathbf{e}_{1}=[1,0, \cdots, 0]^{T}$ ). In the sce- 168 nario that $U_{u}$ is selected, the signal observed at the $N_{r}$ receive 169 antennas is given by

$$
\mathbf{y}=\mathbf{H}_{u} \mathbf{x}+\mathbf{n}
$$

where $\mathbf{H}_{u}$ is the $\left(N_{r} \times L\right)$-element TAS matrix correspond- 171 ing to the selected TA set $U_{u}$, and $\mathbf{n}$ is the $\left(N_{r} \times 1\right)$-element 172 noise vector. The elements of the noise vector $\mathbf{n}$ are complex 173 Gaussian random variables obeying $\mathrm{eN}\left(0, N_{0}\right)$.

The receiver performs maximum-likelihood (ML) detection 175 over all legitimate $\mathrm{SM}$ symbols $\mathbf{x} \in \mathbb{C}^{L \times 1}$ to obtain

$$
\hat{\mathbf{x}}=\arg \min _{\mathbf{x} \in \mathbb{X}}\left\|\mathbf{y}-\mathbf{H}_{u} \mathbf{x}\right\|_{F}^{2}=\arg \min _{\mathbf{x} \in \mathbb{X}}\left\|\mathbf{y}-\mathbf{h}_{u}(q) s_{l}^{q}\right\|_{F}^{2},
$$

where $\mathbb{X}$ is the set of all legitimate transmit symbols and $\mathbf{h}_{u}(q) \quad 177$ is the qth column of the equivalent channel matrix $\mathbf{H}_{u}$. The 178 complexity of the single-stream ML detection of Eq. (4) is low, 179 since a single TA is activated during any time slot [34], [35]. 


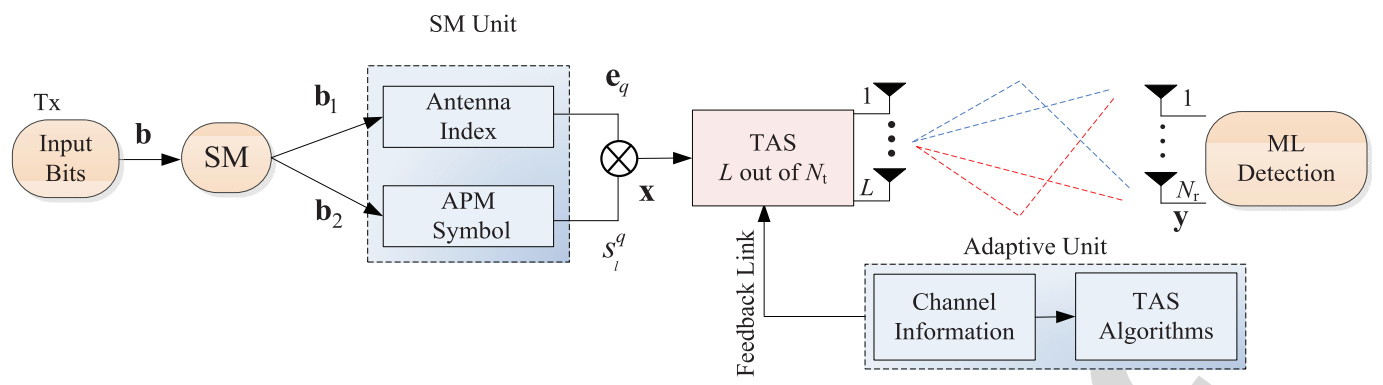

Fig. 1. The system model of the TAS-based SM system.

\section{Conventional TAS Algorithms}

This section offers a brief state-of-the-art review of the existing TAS algorithms proposed for SM systems.

\section{A. The Maximum-Capacity and The Maximum-Norm Based TAS Algorithms}

The capacity $C_{u}$ of the SM-aided MIMO system depends on the classic transmitted signal $s_{l}^{q}$ and the TA index signal $\mathbf{e}_{q}$. As shown in [21], [33], the capacity $C_{\mathrm{s}}$ relying on the signal $s_{l}^{q}$ and the channel $\mathbf{H}_{u}$ is lower bounded by

$$
\alpha=\frac{1}{L} \sum_{i=1}^{L} \log _{2}\left(1+\rho\left\|\mathbf{h}_{u}(i)\right\|_{F}^{2}\right) \leq C_{\mathrm{s}},
$$

where $\mathbf{h}_{u}(i)$ is the ith column of $\mathbf{H}_{u}$ and $\rho$ is the average SNR at the receiver. Moreover, the capacity $C_{\mathrm{TA}}$ relying on the signal $\mathbf{e}_{q}$ is bounded by $C_{\mathrm{TA}} \leq \log _{2}(L)$ [33]. It is proved in [33] that the total capacity $C_{u}=C_{\mathrm{TA}}+C_{\mathrm{s}}$ is bounded by

$$
\alpha \leq C_{u} \leq \alpha+\log _{2}(L),
$$

Based on the bound of Eq. (6), a maximum-capacity based TAS algorithm was formulated in [21] as

$$
\mathbf{H}_{\hat{u}}=\arg \max _{u \in\left\{1, \cdots, N_{U}\right\}} \alpha .
$$

Based on Eq. (5), the optimization objective $\alpha$ of Eq. (7) is maximized by selecting the $L$ TAs associated with the largest channel norms out of the $N_{t}$ TAs, which is equivalent to the maximum-norm based TAS [13] given by

$$
\mathbf{H}_{\hat{u}}=\arg \max _{u \in\left\{1, \cdots, N_{U}\right\}}\left\|\mathbf{H}_{u}\right\|_{F}^{2} .
$$

\section{B. The Exhaustive Max- $d_{\min }$ Based ED-TAS}

In order to improve the BER performance of SM, the free distance (FD) $d_{\min }$ was optimized in [21]. For a given channel $\mathbf{H}_{u}$, its FD can be formulated as

$$
\begin{aligned}
d_{\min }\left(\mathbf{H}_{u}\right) & =\min _{\substack{\mathbf{x}_{i}, \mathbf{x}_{j} \in \mathbb{X} \\
\mathbf{x}_{i} \neq \mathbf{x}_{j}}}\left\|\mathbf{H}_{u}\left(\mathbf{x}_{i}-\mathbf{x}_{j}\right)\right\|_{F}^{2} \\
& =\min _{\mathbf{e}_{i j} \in \mathbb{E}}\left\|\mathbf{H}_{u} \mathbf{e}_{i j}\right\|_{F}^{2}=\min _{\mathbf{e}_{i j} \in \mathbb{E}} \mathbf{e}_{i j}^{H} \mathbf{H}_{u}^{H} \mathbf{H}_{u} \mathbf{e}_{i j},
\end{aligned}
$$

where we have the error vector $\mathbf{e}_{i j}=\mathbf{x}_{i}-\mathbf{x}_{j}, \mathbf{x}_{i}, \mathbf{x}_{j} \in \mathbb{X}$. In 204 [21], the $\max -d_{\min }$ aided ED-TAS algorithm is defined as

$$
\mathbf{H}_{\hat{u}}=\arg \max _{u \in\left\{1, \cdots, N_{U}\right\}} d_{\min }\left(\mathbf{H}_{u}\right) .
$$

The optimum solution obeying the objective function of 206 Eq. (10) can be found by an exhaustive search over all possi- 207 ble $\left(\begin{array}{c}N_{t} \\ L\end{array}\right)$ candidate channel matrices and all the different error 208 vectors, which imposes a complexity order of $\mathcal{O}\left(N_{t}^{2} M^{2}\right)$. This 209 results in an excessive complexity, when high data rates are 210 required.

\section{The Conventional QRD-Based ED-TAS}

In order to reduce the complexity of the exhaustive ED-TAS 213 of Eq. (10), in [21] an ED-TAS based on an equivalent decision 214 metric $\mathbf{D}(u)$ was formulated as:

$$
\mathbf{H}_{\hat{u}}=\arg \max _{u \in\left\{1, \cdots, N_{U}\right\}}\{\min [\mathbf{D}(u)]\},
$$

where $\mathbf{D}(u)$ is an $(L \times L)$-element sub-matrix of an upper tri- 216 angular $\left(N_{t} \times N_{t}\right)$-element matrix $\mathbf{D}$ obtained by deleting the 217 specific rows and columns that are absent in $u$, while $\min [\mathbf{D}(u)] 218$ is the minimum non-zero value of $\mathbf{D}(u)$. Here, the $(i, j)-t h 219$ element of $\mathbf{D}$ can be expressed as

$$
\begin{aligned}
\mathbf{D}_{i j} & =\min _{s_{1}, s_{2} \in \mathbb{S}}\left\|\mathbf{H}\left(s_{1} \mathbf{e}_{i}-s_{2} \mathbf{e}_{j}\right)\right\|_{F}^{2} \\
& =\min _{s_{1}, s_{2} \in \mathbb{S}}\left\|\mathbf{h}(i) s_{1}-\mathbf{h}(j) s_{2}\right\|_{F}^{2},
\end{aligned}
$$

where $s_{1}$ and $s_{2}$ are $M$-ary APM constellation points, 221 while $\mathbf{h}(i)$ and $\mathbf{h}(j)$ are the $i$ th and $j$ th columns of 222 H. Provided that we have $i=j$ in Eq. (12), the corre- 223 sponding element becomes $\mathbf{D}_{i i}=\min _{s_{1}, s_{2} \in \mathbb{S}}\left(\|\mathbf{h}(i)\|_{F}^{2}\left|s_{1}-s_{2}\right|^{2}\right)=224$ $d_{\mathrm{min}}^{\mathrm{APM}}\|\mathbf{h}(i)\|_{F}^{2}$, where $d_{\mathrm{min}}^{\mathrm{APM}}$ is the minimum distance of the 225 APM constellation. For the case of $i \neq j, \mathbf{D}_{i j}$ is re-formulated 226 in the real-valued representation of the QRD as

$$
\mathbf{D}_{i j}=\min _{\substack{s_{1 I}, s_{2 I} \in \mathcal{R}\{\mathbb{S}\}, s_{1 Q}, s_{2 Q} \in \mathcal{J}\{\mathbb{S}\}}}\left\|\mathbf{R}\left[s_{1 I}, s_{1 Q},-s_{2 I},-s_{2 Q}\right]^{T}\right\|_{F}^{2},
$$

where we have $s_{n I}=\mathcal{R}\left\{s_{n}\right\}$ and $s_{n} Q=\mathcal{J}\left\{s_{n}\right\}$ for $n=1,2,228$ while $\mathbf{R}$ is a $(4 \times 4)$-element upper triangular matrix created 229 by the QRD of the resultant channel matrix [21]. As shown in 230 [21], the complexity order of this QRD-TAS is $\mathcal{O}\left(N_{t}^{2} M\right)$, which 231 
increases only linearly with the modulation order $M$. In [22] and [24], both the modulus and the symbol set symmetry of the APM constellations were exploited for further reducing the complexity of this algorithm.

\section{The Conventional SVD-Based ED-TAS}

Although the QRD-based ED-TAS of Eq. (13) is capable of finding the optimal solution, its complexity imposed is a function of the modulation order $M$. Moreover, the high-complexity QRD has to be applied to the $\left(2 N_{r} \times 4\right)$-element channel matrices [21], [22], [24]. Hence, the complexity of this TAS remains high. This problem was circumvented in [23], where the ED was classified into three categories as follows

$$
d_{\min }\left(\mathbf{H}_{u}\right)=\min \left\{d_{\min }^{\text {signal }}, d_{\min }^{\text {spatial }}, d_{\min }^{\text {joint }}\right\}
$$

where we have

$$
\begin{aligned}
d_{\text {min }}^{\text {signal }} & =\min _{\substack{i=1, \cdots, L \\
\text { APM }}}\left\|\mathbf{h}_{u}(i)\right\|_{F}^{2} \min _{s_{a} \neq s_{b} \in \mathbb{S}}\left|s_{a}-s_{b}\right|^{2} \\
& =d_{\min }^{\mathrm{APM}} \operatorname{h}_{u}(i) \|_{F}^{2}, \\
d_{\min }^{\text {spatial }} & =\min _{\substack{i, j=1, \cdots, L \\
i \neq j}}\left\|\mathbf{h}_{u}(i)-\mathbf{h}_{u}(j)\right\|_{F}^{2} \min _{s_{l} \in \mathbb{S}}\left|s_{l}\right|^{2} \\
& =d_{\min }^{\text {Modulus }} \min _{\substack{i, j=1, \cdots, L \\
i \neq j}}\left\|\mathbf{h}_{u}(i)-\mathbf{h}_{u}(j)\right\|_{F}^{2}, \\
d_{\text {min }}^{\text {joint }} & =\min _{\substack{i, j=1, \cdots, L, i \neq j \\
s_{a}, s_{b}, \in \mathbb{S}, a \neq b}}\left\|\mathbf{h}_{u}(i) s_{a}-\mathbf{h}_{u}(j) s_{b}\right\|_{F}^{2} .
\end{aligned}
$$

In Eq. (16), the term $d_{\min }^{\text {Modulus }}=\min _{s_{l} \in \mathbb{S}}\left|s_{l}\right|^{2}$ is the minimum squared modulus value of the APM constellation. Since the calculations of $d_{\mathrm{min}}^{\text {signal }}$ and $d_{\mathrm{min}}^{\text {spatial }}$ in Eqs. (15) and (16) do not depend on the size of APM constellation and the corresponding complexity is low, the complexity of computing the FD of Eq. (14) is dominated by the computation of $d_{\mathrm{min}}^{\text {joint }}$ in Eq. (17). To reduce this complexity, in [23] the Rayleigh-Ritz theorem was utilized for driving a lower bound of $d_{\min }^{\text {joint }}$ as

$$
\begin{aligned}
d_{\min }^{\text {joint }} & =\min _{\substack{i, j=1, \cdots, L, i \neq j \\
s_{a}, s_{b} \in \mathbb{S}, a \neq b}}\left\|\left[\mathbf{h}_{u}(i),-\mathbf{h}_{u}(j)\right]\left[s_{a}, s_{b}\right]^{T}\right\|_{F}^{2} \\
\geq & d_{\min }^{\mathrm{SVD}-\text { bound }} \\
& =\min _{i, j=1, \cdots, L, i \neq j} \lambda_{\min }^{2}\left(\mathbf{H}_{u, i j}\right) \min _{s_{a}, s_{b} \in \mathbb{S}}\left\|\left[s_{a}, s_{b}\right]^{T}\right\|_{F}^{2} \\
& =\min _{i, j=1, \cdots, L, i \neq j} \lambda_{\min }^{2}\left(\mathbf{H}_{u, i j}\right) d_{\min }^{\text {all }}
\end{aligned}
$$

where we have $d_{\min }^{\text {all }}=\min _{s_{a}, s_{b} \in \mathbb{S}}\left\|\left[s_{a}, s_{b}\right]^{T}\right\|_{F}^{2}$ and $\mathbf{H}_{u, i j}=$ $\left[\mathbf{h}_{u}(i),-\mathbf{h}_{u}(j)\right]$ is an $\left(N_{r} \times 2\right)$-element matrix. Here, $\lambda_{\min }^{2}\left(\mathbf{H}_{u, i j}\right)$ is the minimum squared singular value of the submatrix $\mathbf{H}_{u, i j}$. Upon exploiting Eq. (18), the distance $d_{\min }\left(\mathbf{H}_{u}\right)$ of Eq. (14) is bounded by

$$
d_{\min }^{\mathrm{SVD}}\left(\mathbf{H}_{u}\right)=\min \left\{d_{\min }^{\mathrm{signal}}, d_{\mathrm{min}}^{\text {spatial }}, d_{\min }^{\mathrm{SVD}-\text { bound }}\right\} .
$$

Based on Eq. (19), the SVD-TAS algorithm is given by

$$
\mathbf{H}_{\hat{u}}=\arg \max _{u \in\left\{1, \cdots, N_{U}\right\}} d_{\min }^{\mathrm{SVD}}\left(\mathbf{H}_{u}\right) .
$$

Compared to the conventional QRD-based TAS, this bound- 259 aided algorithm has the following advantages:

- Using the SVD-based bound of Eq. (18), the calcula- 261 tion of the distance $d_{\min }^{\text {joint }}$ is independent of the APM 262 modulation order;

- Moreover, the SVD operation of Eq. (18) is performed 264 on the smaller channel matrices of size $\left(N_{r} \times 2\right)$ com- 265 pared to the QRD-based ED-TAS, which is performed on 266 $\left(2 N_{r} \times 4\right)$-element matrices. In [25], the complexity of 267 SVD-TAS [23] was further reduced through an alternative 268 computation of the singular value.

\section{The Proposed LOW-COMPLEXITY ED-TAS}

As shown in subsection III, the conventional QRD-based 271 ED-TAS is capable of achieving the optimal BER, but it 272 imposes high complexity. In contrast, the SVD-based ED-TAS 273 imposes a lower complexity at the cost of a BER performance 274 degradation, because the derived bound may be loose and the 275 corresponding TAS results may be suboptimal. 276

To circumvent this problem, in this section, a pair of ED-TAS 277 algorithms are proposed. Specifically, an improved QRD-TAS 278 is proposed, where a tighter QRD-based lower bound of the 279 ED is found for replacing the SVD-based bound of [23], while 280 the sparse nature ${ }^{1}$ of the error vectors of SM is exploited to 281 avoid the full-dimensional QRD operation. Then, for striking 282 a further flexible BER vs complexity tradeoff, we propose an 283 EVM-based ED-TAS algorithm, which exploits the error vector 284 selection probability to shrink the search space.

\section{A. The Proposed QRD-Based ED-TAS}

1) The QRD-Based Bounds: To evaluate the value of $d_{\min }^{\text {joint }} 287$ more accurately, in this paper, we apply the QRD-based bound 288 to replace the SVD-bound of Eq. (18). Specifically, the sub- 289 matrix $\mathbf{H}_{u, i j}$ of Eq. (18) is first subjected to the QRD [38], 290 yielding $\mathbf{H}_{u, i j}=\tilde{\mathbf{Q}} \tilde{\mathbf{R}}$, where $\tilde{\mathbf{Q}}$ is an $\left(N_{r} \times 2\right)$ column-wise 291 orthonormal matrix and $\tilde{\mathbf{R}}$ is a $(2 \times 2)$ upper triangular matrix 292 with positive real-valued diagonal entries formulated as

$$
\tilde{\mathbf{R}}=\left[\begin{array}{cc}
\tilde{R}_{1,1} & \tilde{R}_{1,2} \\
0 & \tilde{R}_{2,2}
\end{array}\right]
$$

Let $[\tilde{\mathbf{R}}]_{k}=\tilde{R}_{k, k}$ denote the $k t h$ diagonal entry of $\tilde{\mathbf{R}}$. Based 294 on this decomposition, another lower bound of the distance 295 $d_{\min }^{\text {joint }}$ in Eq. (18) can be formulated as

$$
\begin{aligned}
d_{\min }^{\text {joint }} & \geq d_{\min }^{\mathrm{QRD}-\text { bound }} \\
& =\min _{i, j=1, \cdots, L, i \neq j}\left\{[\tilde{\mathbf{R}}]_{\min }^{2}\right\} \min _{s_{a} \neq s_{b} \in \mathbb{S}}\left\|\left[s_{a}, s_{b}\right]\right\|_{F}^{2}, \\
& =\min _{i, j=1, \cdots, L, i \neq j}\left\{[\tilde{\mathbf{R}}]_{\min }^{2}\right\} d_{\min }^{\text {all }}
\end{aligned}
$$

\footnotetext{
${ }^{1}$ In SM, the transmit vector $\mathbf{x}$ only has a single non-zero element, hence the number of non-zero elements of the error vectors $\mathbf{e}_{i j}$ of SM is no more than 2.
} 
where $[\tilde{\mathbf{R}}]_{\min }^{2}$ is the minimum squared nonzero diagonal entry of the upper matrix $\tilde{\mathbf{R}}$, given by

$$
[\tilde{\mathbf{R}}]_{\min }=\min \left\{\tilde{R}_{1,1}, \tilde{R}_{2,2}\right\} .
$$

Lemma 1: For an $\left(N_{r} \times 2\right)$-element full column-rank matrix $\mathbf{H}_{u, i j}$ associated with its minimum squared singular non-zero value $\lambda_{\min }^{2}\left(\mathbf{H}_{u, i j}\right)$ for SVD and its minimum squared diagonal non-zero entry $[\tilde{\mathbf{R}}]_{\min }^{2}$ of $\tilde{\mathbf{R}}$ for $\mathrm{QRD}$, respectively, the inequality $[\tilde{\mathbf{R}}]_{\min }^{2} \geq \lambda_{\min }^{2}\left(\mathbf{H}_{u, i j}\right)$ is satisfied.

According to the analysis process in Section III of [38], the formulation of Lemma 1 is straightforward. As a result, the lower bound of Eq. (22) achieved by the QRD is tighter than that of the SVD algorithm in Eq. (18).

To derive an even tighter upper QRD bound than that of Eq. (22), the permutation matrix $\Pi_{m}$ can be invoked for calculating $d_{\min }^{\text {joint }}$ of Eq. (22) as

$$
d_{\min }^{\text {joint }}=\min _{\substack{i, j=1, \cdots, L, i \neq j, s_{a}, s_{b} \in \mathbb{S}}}\left\|\left[\mathbf{h}_{u}(i),-\mathbf{h}_{u}(j)\right] \boldsymbol{\Pi}_{m} \boldsymbol{\Pi}_{m}^{-1}\left[s_{a}, s_{b}\right]^{T}\right\|_{F}^{2},
$$

where $\boldsymbol{\Pi}_{m}$ is an orthogonal matrix satisfying $\boldsymbol{\Pi}_{m}^{-1}=\boldsymbol{\Pi}_{m}^{T}$. Since the size of the channel matrix $\mathbf{H}_{u, i j}=\left[\mathbf{h}_{u}(i),-\mathbf{h}_{u}(j)\right]$ is $N_{r} \times 2$, we only have two legitimate permutation matrices $\Pi_{m} \in \mathbb{C}^{2 \times 2}, m=1,2$, namely

$$
\boldsymbol{\Pi}_{1}=\left[\begin{array}{ll}
1 & 0 \\
0 & 1
\end{array}\right] \text { and } \boldsymbol{\Pi}_{2}=\left[\begin{array}{ll}
0 & 1 \\
1 & 0
\end{array}\right] .
$$

For each matrix $\Pi_{m}$, similar to Eq. (22), the corresponding QRD-based bound is

$$
\begin{aligned}
d_{\min }^{\text {joint }} & \geq \min _{i, j=1, \cdots, L, i \neq j}\left\{\left[\tilde{\mathbf{R}}_{m}\right]_{\min }^{2}\right\} \min _{s_{a}, s_{b} \in \mathbb{S}}\left\|\boldsymbol{\Pi}_{m}^{T}\left[s_{a}, s_{b}\right]^{T}\right\|_{F}^{2} \\
& =\left[\tilde{\mathbf{R}}_{m}\right]_{\min }^{2} d_{\min }^{\text {all }},
\end{aligned}
$$

where $\tilde{\mathbf{R}}_{m}$ is the upper triangular part of the QRD of the equivalent matrix $\mathbf{H}_{u, i j} \boldsymbol{\Pi}_{m}$. Note in Eq. (26) that the permutation matrix does not change the distance of $\left\|\boldsymbol{\Pi}_{m}^{T}\left[s_{a}, s_{b}\right]\right\|_{F}^{2}$ and we have $\min _{s_{a}, s_{b} \in \mathbb{S}}\left\|\Pi_{m}^{T}\left[s_{a}, s_{b}\right]^{T}\right\|_{F}^{2}=$ $\min _{s_{a}, s_{b} \in \mathbb{S}}\left\|\left[s_{a}, s_{b}\right]^{T}\right\|_{F}^{2}=d_{\text {min }}^{\text {all }}$. For the permutation matrices given in Eq. (25), we can obtain two different values $\left[\tilde{\mathbf{R}}_{m}\right]_{\min }$ $(m=1,2)$, which are given by $\left[\tilde{\mathbf{R}}_{1}\right]_{\min }=\min \left\{\tilde{R}_{1,1}\left(\boldsymbol{\Pi}_{1}\right)\right.$, $\left.\tilde{R}_{2,2}\left(\boldsymbol{\Pi}_{1}\right)\right\}$ and $\left[\tilde{\mathbf{R}}_{2}\right]_{\min }=\min \left\{\tilde{R}_{1,1}\left(\boldsymbol{\Pi}_{2}\right), \tilde{R}_{2,2}\left(\boldsymbol{\Pi}_{2}\right)\right\}$. Here, $\tilde{R}_{1,1}\left(\boldsymbol{\Pi}_{m}\right)$ and $\tilde{R}_{2,2}\left(\boldsymbol{\Pi}_{m}\right), m=1,2$ are the diagonal elements of $\tilde{\mathbf{R}}_{m}$.

Remark: The bound of Eq. (22) constitutes a special case of the bound of Eq. (26), which can be obtained by setting $m=1$.

Based on Eq. (26), an improved QRD-based upper bound of the distance $d_{\min }^{\text {joint }}$ is given by

$$
\begin{aligned}
d_{\min }^{\text {joint }} & \geq d_{\min }^{\mathrm{QRD}} \\
& =\min _{i, j=1, \cdots, L, i \neq j}\left\{\left[\tilde{\mathbf{R}} Q R Q_{-} P\right]_{\min }^{2}\right\} d_{\min }^{\text {all }} .
\end{aligned}
$$

where we have $\left[\tilde{\mathbf{R}}_{Q R Q_{-} P}\right]_{\min }^{2}=\max \left\{\left[\tilde{\mathbf{R}}_{1}\right]_{\min }^{2},\left[\tilde{\mathbf{R}}_{2}\right]_{\min }^{2}\right\}$.

Lemma 2: For an $\left(N_{r} \times 2\right)$-element full column-rank 332 matrix $\mathbf{H}_{u, i j}$ having a minimum squared diagonal non-zero 333 entry $[\tilde{\mathbf{R}}]_{\min }^{2}$ for its $\mathrm{QRD}$ and a value of $\left[\tilde{\mathbf{R}}_{Q R Q_{-} P}\right]_{\min }^{2}=334$ $\max \left\{\left[\tilde{\mathbf{R}}_{1}\right]_{\min }^{2},\left[\tilde{\mathbf{R}}_{2}\right]_{\min }^{2}\right\}$ based on the pair of legitimate permuta- 335 tion matrices $\boldsymbol{\Pi}_{m} \in \mathbb{C}^{2 \times 2}, m=1,2$, respectively, the inequal- 336 ity $\left[\tilde{\mathbf{R}}_{Q R Q_{-} P}\right]_{\min }^{2} \geq[\tilde{\mathbf{R}}]_{\min }^{2}$ is satisfied.

Since we have $[\tilde{\mathbf{R}}]_{\min }^{2}=\left[\tilde{\mathbf{R}}_{1}\right]_{\min }^{2}$, Lemma 2 can be obtained. 338

2) The Proposed QRD-Based ED-TAS: According to 339 Lemma 2, the QRD bound of Eq. (27) is tighter than that 340 of Eq. (22). Hence, we use this tighter bound to derive the 341 proposed QRD-based ED-TAS as

$$
\mathbf{H}_{\hat{u}}=\arg \max _{u \in\left\{1, \cdots, N_{U}\right\}}\left\{d_{\min }^{\text {signal }}, d_{\min }^{\text {spatial }}, d_{\min }^{\mathrm{QRD}-\text { bound_P }}\right\} .
$$

Note that the complexity of the QRD-based TAS is domi- 343 nated by the computation of $\left[\tilde{\mathbf{R}}_{m}\right]_{\text {min. }}$. In general, the full QRD 344 can be adopted in Eq. (26) for solving Eq. (27). However, this 345 may impose a high complexity. In order to reduce this com- 346 plexity, for a fixed channel $\mathbf{H}_{u, i j}$, we found that the value of 347 $\left[\tilde{\mathbf{R}}_{m}\right]_{\text {min }}$ only depends on the diagonal entries of $\tilde{\mathbf{R}}_{m}$, namely 348 $\tilde{R}_{k, k}\left(\boldsymbol{\Pi}_{m}\right)(k=1,2)$, which can be directly calculated as [38] 349

$$
\left[\tilde{\mathbf{R}}_{m}\right]_{k}=\tilde{R}_{k, k}\left(\boldsymbol{\Pi}_{m}\right)=\sqrt{\frac{\operatorname{det}\left[(\mathbf{G}(1: k))^{H} \mathbf{G}(1: k)\right]}{\operatorname{det}\left[(\mathbf{G}(1: k-1))^{H} \mathbf{G}(1: k-1)\right]}},
$$

where $\mathbf{G}(1: k)$ denotes a matrix consisting of the first $k 350$ columns of $\mathbf{H}_{u, i j} \boldsymbol{\Pi}_{m}$. In the classic V-BLAST systems, the cal- 351 culation of Eq. (29) suffers from the problem of having a high 352 complexity [38]. In SM, the number of non-zero elements of 353 the error vectors of SM is up to 2. This sparse character leads 354 to the simple sub-matrix $\mathbf{H}_{u, i j}=\left[\mathbf{h}_{u}(i),-\mathbf{h}_{u}(j)\right] \in \mathbb{C}^{N_{r} \times 2}$ and 355 hence the values of $\tilde{R}_{k, k}\left(\boldsymbol{\Pi}_{m}\right)(m=1,2, k=1,2)$ are given by 356

$$
\begin{aligned}
& \tilde{R}_{1,1}\left(\boldsymbol{\Pi}_{1}\right)=\sqrt{\left\|\mathbf{h}_{u}(i)\right\|_{F}^{2}}, \\
& \tilde{R}_{2,2}\left(\boldsymbol{\Pi}_{1}\right)=\sqrt{\frac{\left\|\mathbf{h}_{u}(i)\right\|_{F}^{2}+\left\|\mathbf{h}_{u}(j)\right\|_{F}^{2}-2 \mathcal{R}\left\{\mathbf{h}_{u}(i)^{H} \mathbf{h}_{u}(j)\right\}}{\left\|\mathbf{h}_{u}(i)\right\|_{F}^{2}}} \\
& \tilde{R}_{1,1}\left(\boldsymbol{\Pi}_{2}\right)=\sqrt{\left\|\mathbf{h}_{u}(j)\right\|_{F}^{2}}
\end{aligned}
$$

and

$$
\tilde{R}_{2,2}\left(\boldsymbol{\Pi}_{2}\right)=\sqrt{\frac{\left\|\mathbf{h}_{u}(i)\right\|_{F}^{2}+\left\|\mathbf{h}_{u}(j)\right\|_{F}^{2}-2 \mathcal{R}\left\{\mathbf{h}_{u}(i)^{H} \mathbf{h}_{u}(j)\right\}}{\left\|\mathbf{h}_{u}(j)\right\|_{F}^{2}}}
$$

The complexity of our proposed QRD-TAS of Eq. (28) 358 is dominated by the computation of $\tilde{R}_{k, k}\left(\boldsymbol{\Pi}_{m}\right), m=1,2$. In 359 $\mathrm{SM}$, these values only depend on the values of $\left\|\mathbf{h}_{u}(i)\right\|_{F}^{2}, \quad 360$ $\left\|\mathbf{h}_{u}(j)\right\|_{F}^{2}$ and $\mathbf{h}_{u}(i){ }^{H} \mathbf{h}_{u}(j)$, which are elements of the matrix 361 $\mathbf{H}^{H} \mathbf{H}$, as shown in Eqs. (30)-(33). Based on this observa- 362 tion, we can calculate the values of $\tilde{R}_{k, k}\left(\boldsymbol{\Pi}_{m}\right), m=1,2$ by 363 reusing these elements for the different TAS candidates $\mathbf{H}_{u}, 364$ 
TABLE I

COMPleXity COMPARISON OF DIFFERENT TAS ALgORITHMS FOR SM SySTEMS

\begin{tabular}{|l|r|r|}
\hline TAS algorithm & ED Optimality & Computational complexity \\
\hline \hline Exhaustive ED-TAS [13] & optimal & $\frac{N_{t}\left(N_{t}-1\right)}{2}\left(5 N_{r}-1\right) M^{2}$ \\
\hline $\begin{array}{l}\text { Maximum-norm based } \\
\text { TAS of [21] }\end{array}$ & sub-optimal & $2 N_{t} N_{r}-N_{t}$ \\
\hline $\begin{array}{l}\text { Minimum-correlation } \\
\text { based TAS of [15] }\end{array}$ & sub-optimal & $2 N_{t}^{2} N_{r}-N_{t}^{2}+\frac{3}{2} N_{t}\left(N_{t}-1\right)$ \\
\hline $\begin{array}{l}\text { Conventional QRD-based } \\
\text { ED-TAS of [24] }\end{array}$ & optimal & $\begin{array}{c}2 N_{t} N_{r}-N_{t}+32 N_{t}\left(N_{t}-1\right)\left(N_{r}-\frac{2}{3}\right) \frac{M}{N_{A P M}} \\
\left(N_{A P M}=M \text { for PSK, } N_{A P M}=4 \text { for QAM }\right)\end{array}$ \\
\hline $\begin{array}{l}\text { Conventional SVD-based } \\
\text { ED-TAS of [23] }\end{array}$ & sub-optimal & $2 N_{t} N_{r}-N_{t}+\frac{19}{2} N_{t}\left(N_{t}-1\right)\left(N_{r}-\frac{1}{3}\right)$ \\
\hline Simplified SVD-TAS [25] & sub-optimal & $\frac{N_{t}\left(N_{t}-1\right)}{2}\left(2 N_{r}+11\right)+N_{t}\left(2 N_{r}-1\right)$ \\
\hline $\begin{array}{l}\text { Proposed QRD-based } \\
\text { ED-TAS }\end{array}$ & sub-optimal & $2 N_{t}^{2} N_{r}+\frac{3}{2} N_{t}\left(N_{t}-1\right)$ \\
\hline $\begin{array}{l}\text { Proposed EVM-based } \\
\text { ED-TAS }\end{array}$ & $M$-PSK: optimal & $2 N_{t}^{2} N_{r}-N_{t}^{2}+\frac{1}{2} N_{t}\left(N_{t}-1\right)(M+7)$ \\
\hline Exhaustive TAS\&PA & $M-\mathrm{QAM}\left\{\begin{array}{r}\text { sub-optimal, } K<v \\
\text { optimal, } K=v\end{array}\right.$ & $2 N_{t}^{2} N_{r}-N_{t}^{2}+\frac{15}{2} G N_{t}\left(N_{t}-1\right)$ \\
\hline Low-complexity TAS\&PA & - & $\left(\begin{array}{c}N_{t} \\
L\end{array}\right) C_{\mathrm{PA}}$ \\
\hline
\end{tabular}

hence the resultant complexity is considerably reduced compared to the conventional QRD-based ED-TAS, as will show in Table I.

To confirm the benefits of the QRD-based bound derived in Eq. (27), Fig. 2 shows the BER performance of the proposed QRD-based ED-TAS algorithm in contrast to the existing SVDbased ED-TAS of [23]. Moreover, we add the performance of the norm-based TAS of [13] and of the exhaustive-search based optimal ED-TAS of [21] as benchmarks. In Fig. 2, the number of TAs is set to $N_{t}=4$, where $L=2$ out of $N_{t}=$ 4 TAs were selected in these TAS algorithms. As expected, since the proposed QRD-based ED-TAS has a tighter bound, in Fig. 2 it performs better than the SVD-based ED-TAS. Quantitatively, observe in Fig. 2 that this scheme provides an SNR gain of about $1.2 \mathrm{~dB}$ over the SVD-based ED-TAS at the BER of $10^{-5}$. In Fig. 2, we also observe that the QRDbased ED-TAS achieves a near-optimum performance, where the performance gap between the proposed QRD-based EDTAS and the exhaustive-search-based optimal ED-TAS is only about $0.2 \mathrm{~dB}$. We will provide more detailed comparisons about the BER and the complexity issues in Section VI.

\section{B. The Proposed EVM-Based ED-TAS}

In this section, for striking a further flexible tradeoff in terms of the BER attained and the complexity imposed, we propose an EVM-based ED-TAS algorithm. The proposed EVM-TAS directly calculates the value of $d_{\min }\left(\mathbf{H}_{u}\right)$ for the specific TAS candidate $\mathbf{H}_{u}$, rather than exploiting the equivalent decision metric of Eq. (13) or the estimated bound of (18). Specifically, we will derive simple optimization metrics for both PSK and QAM constellations, where the error-vector selection probability is exploited for reducing the search space.

1) The Calculation of $d_{\min }\left(\mathbf{H}_{u}\right)$ in EVM-Based ED-TAS: Specifically, the $M$-PSK constellation can be expressed as $\mathbb{S}_{P S K}=\left\{e^{j \frac{2 m \pi}{M}}, m=0, \cdots, M-1\right\}$, and the symbols of the rectangular $M=4^{k} \mathrm{QAM}$ constellation belong to the set of [36]

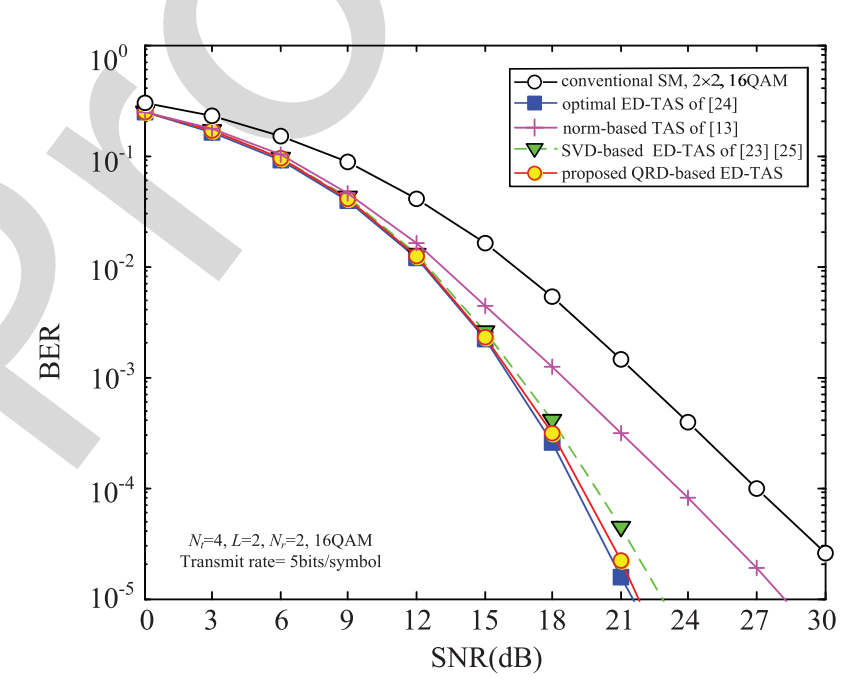

Fig. 2. BER performance comparison of the existing TAS algorithms and the proposed QRD-based ED-TAS algorithm. The setup of the simulation is based on $N_{t}=4, N_{r}=2, L=2$ and 16-QAM. The transmit rate is 5 bits/symbol.

$$
\mathbb{S}_{Q A M}=\frac{1}{\sqrt{\beta_{k}}}\{a+b j, a-b j,-a+b j,-a-b j\},
$$

where we have $\beta_{k}=\frac{2}{3}\left(4^{k}-1\right)$ and $a, b \in\left\{1,3, \cdots, 2^{k}-1\right\} .401$ Similar to Eq. (14), the calculation of $d_{\min }\left(\mathbf{H}_{u}\right)$ is parti- 402 tioned into three cases: $d_{\min }^{\text {signal }}, d_{\text {min }}^{\text {spatial }}$ and $d_{\text {min }}^{\text {joint }}$. As shown in 403 Eqs. (15)-(16), $d_{\mathrm{min}}^{\text {signal }}$ depends the minimum distance of the 404 $\mathrm{APM} d_{\min }^{\mathrm{APM}}$ as [39]

$$
d_{\mathrm{min}}^{\mathrm{APM}}=\left\{\begin{array}{lll}
4 \sin ^{2}(\pi / M) & \text { for } & M-\mathrm{PSK} \\
\frac{4}{\beta_{k}} & \text { for } & M-\mathrm{QAM}
\end{array},\right.
$$

while $d_{\text {min }}^{\text {spatial }}$ relies on the minimum squared modulus value 406 $d_{\mathrm{min}}^{\text {Modulus }}$ of the APM constellation as

$$
d_{\min }^{\text {Modulus }}=\left\{\begin{array}{lll}
1 & \text { for } & M-\mathrm{PSK} \\
\frac{2}{\beta_{k}} & \text { for } & M-\mathrm{QAM}
\end{array} .\right.
$$


Based on Eqs. (35) and (36), the complexity of computing the values of $d_{\mathrm{min}}^{\text {signal }}$ and $d_{\mathrm{min}}^{\text {spatial }}$ in Eqs. (15)-(16) may be deemed negligible. Hence, we only have to reduce the complexity of computing $d_{\mathrm{min}}^{\text {joint }}$, which can be achieved as follows:

$$
\begin{aligned}
& d_{\min }^{\text {joint }-E V M}=\min _{\substack{i, j=1, \cdots, L, i \neq j \\
s_{a}, s_{b} \in \mathbb{S}}}\left\|\mathbf{h}_{u}(i) s_{a}-\mathbf{h}_{u}(j) s_{b}\right\|_{F}^{2} \\
& =\min _{\substack{i, j=1, \cdots, L, i \neq j, s_{a}, s_{b} \in \mathbb{S}}}\left|s_{a}\right|^{2}\left\|\mathbf{h}_{u}(i)\right\|_{F}^{2}+\left|s_{b}\right|^{2}\left\|\mathbf{h}_{u}(j)\right\|_{F}^{2}-2 m_{\mathrm{APM}},
\end{aligned}
$$

where we have $m_{\mathrm{APM}}=\mathcal{R}\left\{s_{a}^{H} s_{b} \mathbf{h}_{u}(i)^{H} \mathbf{h}_{u}(j)\right\}$, which relies on the specific APM scheme adopted. Next, we will derive the simplified metrics $d_{\min }^{\text {joint-EVM }}$ for the general family of $M$-PSK and $M$-QAM modulated SM systems.

2) Simplification for $M$-PSK Schemes: For a pair of $M$-PSK symbols $s_{a}=e^{j \frac{2 a \pi}{M}}$ and $s_{b}=e^{j \frac{2 b \pi}{M}}$, the possible values of $s_{a}^{H} s_{b}$ obey $e^{j \frac{2(b-a) \pi}{M}},(b-a) \in\{-(M-1), \cdots,(M-1)\}$. As a result, $m_{\mathrm{APM}}$ of the general $M$-PSK scheme obeys:

$$
m_{\mathrm{APM}} \in\left\{\mathcal{R}\left\{\mathbf{h}_{u}(i)^{H} \mathbf{h}_{u}(j)\right\} \cos \theta_{n}-\mathcal{J}\left\{\mathbf{h}_{u}(i){ }^{H} \mathbf{h}_{u}(j)\right\} \sin \theta_{n}\right\},
$$

where $\theta_{n}=\frac{2 n \pi}{M}, n=-(M-1), \cdots,(M-1)$. Since the minimum ED is considered in Eq (37), only the maximum value of $m_{\mathrm{APM}}$ needs to be considered, which is given by Eq. (39), shown at the bottom of the page. As shown in Eq. (39), the number of possible $\theta_{n}$ values is reduced from $2 M-1$ to $\frac{M}{4}+1$. According to Eq. (39), $\left|s_{a}\right|^{2}=1$ and $\left|s_{b}\right|^{2}=1$, the distance $d_{\min }^{\text {joint }-E V M}$ of Eq. (37) is simplified for $M$-PSK as follows:

$$
d_{\min }^{\text {joint } E V} \stackrel{\substack { M \\
\begin{subarray}{c}{i, j=1, \cdots, L \\
i \neq j{ M \\
\begin{subarray} { c } { i , j = 1 , \cdots , L \\
i \neq j } }\end{subarray}}{=} \min ,\left\|\mathbf{h}_{u}(i)\right\|_{F}^{2}+\left\|\mathbf{h}_{u}(j)\right\|_{F}^{2}-2 m_{M-\operatorname{PSK}}\left(\mathbf{H}_{u}\right) .
$$

Example: The constellation points $s_{a}$ and $s_{b}$ of BPSK and QPSK modulation schemes belong to the set $\mathbb{S}_{\mathrm{BPSK}}=\{ \pm 1\} \quad$ and $\quad \mathbb{S}_{\mathrm{QPSK}}=\{ \pm 1, \pm j\}$, respectively. Based on Eq. (39), the corresponding optimized metrics $m_{M-\mathrm{PSK}}\left(\mathbf{H}_{u}\right)=\max m_{\mathrm{APM}}$ are simplified to $m_{2-\mathrm{PSK}}\left(\mathbf{H}_{u}\right)=\left|\mathcal{R}\left\{\mathbf{h}_{u}(i)^{H} \mathbf{h}_{u}(j)\right\}\right|$ and $m_{4-\mathrm{PSK}}\left(\mathbf{H}_{u}\right)=$ $\max \left\{\left|\mathcal{R}\left\{\mathbf{h}_{u}(i)^{H} \mathbf{h}_{u}(j)\right\}\right|,\left|\mathcal{J}\left\{\mathbf{h}_{u}(i){ }^{H} \mathbf{h}_{u}(j)\right\}\right|\right\}$, respectively.

As shown in Eqs. (37)-(40), since we have $\left|s_{a}\right|^{2}=1,\left|s_{b}\right|^{2}=$ 1 and a reduced set $s_{a}^{H} s_{b}$ for $M$-PSK constellation, the complexity of calculating $d_{\text {min }}^{\text {joint }-E V M}$ is low, as it will be shown in Table I.

3) Simplification for M-QAM Schemes: When M-QAM constellations are considered, the calculation of $d_{\min }^{\text {joint }-E V M}$ in Eq. (37) becomes substantially complicated, because there are many combinations of the values of $\left|s_{a}\right|^{2},\left|s_{b}\right|^{2}$ and $s_{a}^{H} s_{b}$ in Eq. (37), which lead to different received SM-symbol distances. To derive a simplified optimized metrics for $M$-QAM, we first introduce the following Lemma.

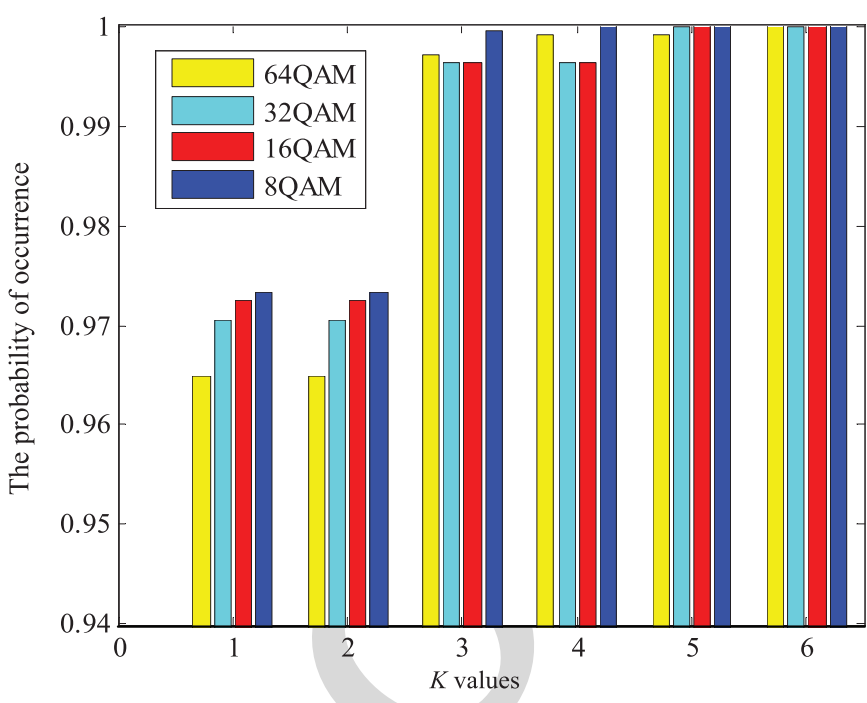

Fig. 3. The statistical probability of the norm error vectors relying on $K$ minimum moduli, yielding the optimal ED-TAS solution, where the system setup is $N_{t}=4, N_{r}=2$ and $L=2$.

Lemma 3: It is highly likely that an error vector associated 445 with a small norm value yields the FD value of Eq. (9). Thus, 446 the search space to be evaluated for finding the FD can be 447 reduced to a few dominant error vectors having small norm 448 values.

Proof: Based on the Rayleigh-Ritz theorem of [37], for 450 a fixed channel matrix $\mathbf{H}_{u, i j}$ and a given error vector $\mathbf{e}_{i j}, 451$ the distance amongst the received symbols is bounded by 452 $\lambda_{\max }^{2}\left(\mathbf{H}_{u, i j}\right)\left\|\mathbf{e}_{i j}\right\|^{2} \geq\left\|\mathbf{H}_{u} \mathbf{e}_{i j}\right\|^{2} \geq \lambda_{\min }^{2}\left(\mathbf{H}_{u, i j}\right)\left\|\mathbf{e}_{i j}\right\|^{2}$, where 453 $\lambda_{\max }^{2}\left(\mathbf{H}_{u, i j}\right)$ is the maximum squared singular value of the sub- 454 matrix $\mathbf{H}_{u, i j}=\left[\mathbf{h}_{u}(i),-\mathbf{h}_{u}(j)\right]$. It may be readily shown that 455 the values of $\lambda_{\max }^{2}\left(\mathbf{H}_{u, i j}\right)$ and $\lambda_{\min }^{2}\left(\mathbf{H}_{u, i j}\right)$ are constants for 456 a fixed channel realization $\mathbf{H}_{u, i j}$, while the value of $\left\|\mathbf{e}_{i j}\right\|^{2} 457$ depends on the specific APM constellation points. Based on the 458 bound above, it is highly likely that an $\mathbf{e}_{i j}$ with a small norm 459 yields low upper bound and lower bound. Hence it has a high 460 probability of generating the FD value, as it will be exemplified 461 in Fig. 3.

462

Based on Lemma 3, for the sake of striking a beneficial 463 trade-off between the BER performance and complexity for 464 $M$-QAM, the search space is limited to the error vectors hav- 465 ing small modulus values and only these vectors are utilized to 466 compute the FD metric. Specifically, we first evaluate all possi- 467 ble modulus values $T_{1}, T_{2}, T_{3}, \cdots, T_{v}$ of all the legitimate error 468 vectors $\mathbf{e}_{i j}$, then we find the $K$ smallest $T_{K}$ from the full set 469 of $\left\{T_{1}, T_{2}, T_{3}, \cdots, T_{v}\right\}$ and only consider the set of $\mathbf{e}_{i j}$ having 470 moduli lower than $T_{K}$ to compute $d_{\min }\left(\mathbf{H}_{u}\right)$. In this process, 471 the error vectors can be divided into the pair of sub-sets $\mathbb{D}_{1}$ and 472 $\mathbb{D}_{2}$ based on their sparsity, where $\mathbb{D}_{1}$ contains the error vectors, 473 which have only a single non-zero element, while $\mathbb{D}_{2}$ contains 474

$$
\begin{aligned}
m_{M-\mathrm{PSK}}\left(\mathbf{H}_{u}\right)=\max _{n} m_{\mathrm{APM}} & =\max _{n \in\{-(M-1), \cdots, M-1\}}\left\{\mathcal{R}\left\{\mathbf{h}_{u}(i)^{H} \mathbf{h}_{u}(j)\right\} \cos \theta_{n}-\mathcal{J}\left\{\mathbf{h}_{u}(i)^{H} \mathbf{h}_{u}(j)\right\} \sin \theta_{n}\right\} \\
& =\max _{n \in\{0, \cdots, M / 4\}}\left\{\left|\mathcal{R}\left\{\mathbf{h}_{u}(i)^{H} \mathbf{h}_{u}(j)\right\} \cos \theta_{n}-\mathcal{J}\left\{\mathbf{h}_{u}(i)^{H} \mathbf{h}_{u}(j)\right\} \sin \theta_{n}\right|\right\}
\end{aligned} .
$$


the error vectors, which have two non-zero elements. As will be shown in our simulation results, $K=3$ is a good choice for diverse configurations, hence we only provide the simplified expressions of $d_{\min }^{\text {joint }-E V M}$ for $K \leq 3$ as follows.

For $K=1$, according to the $M$-QAM constellation of Eq. (34), only error vectors having $T_{1}=\sqrt{\frac{4}{\beta_{k}}}$ are considered and the associated sets $\mathbb{D}_{1}$ and $\mathbb{D}_{2}$ are given by $\mathbb{D}_{1}=\frac{1}{\sqrt{\beta_{k}}}\left\{ \pm 2 \mathbf{e}_{i}, \pm 2 \mathrm{j} \mathbf{e}_{i}\right\}, i=1, \cdots, L \quad$ and $\mathbb{D}_{2}=\frac{1}{\sqrt{\beta_{k}}}\left\{( \pm 1 \pm 1 \mathrm{j}) \mathbf{e}_{i}-( \pm 1 \pm 1 \mathrm{j}) \mathbf{e}_{j}\right\}, i, j=1, \cdots, L, i \neq$ $j$, respectively, where $\mathbf{e}_{i}$ and $\mathbf{e}_{j}$ are the active TA selection vectors in Eq. (2). Since only the minimum ED is considered, the set $\mathbb{D}_{1}$ can be reduced to $\mathbb{D}_{1}=\frac{1}{\sqrt{\beta_{k}}}\left\{2 \mathbf{e}_{i}\right\}, i=1, \cdots, L$. Moreover, based on the set $\mathbb{D}_{2}$, it is find that the elements $s_{a}$ and $s_{b}$ belong to the reduced set $\frac{1}{\sqrt{\beta_{k}}}\{ \pm 1 \pm 1 \mathrm{j}\}$ and we have $\left|s_{a}\right|^{2}=\frac{2}{\beta_{k}},\left|s_{b}\right|^{2}=\frac{2}{\beta_{k}}$ and $s_{a}^{H} s_{b} \in \frac{2}{\beta_{k}}\{ \pm 1, \pm 1 \mathrm{j}\}$. Substituting these values into Eq. (37), we get the simplified optimized metric for $K=1$ as

$$
d_{\min , K=1}^{\text {joint } E V M}=\min \frac{2}{\substack{i, j=1, \cdots, L \\ i \neq j,}},\left\|\mathbf{h}_{u}(i)\right\|_{F}^{2}+\frac{2}{\beta_{k}}\left\|\mathbf{h}_{u}(j)\right\|_{F}^{2}-2 m_{M-Q A M}^{K=1},
$$

where we have

$$
\begin{aligned}
& m_{M-Q A M}^{K=1}=\max m_{\mathrm{APM}} \\
& =\max \left\{\frac{2}{\beta_{k}}\left|\mathcal{R}\left\{\mathbf{h}_{u}(i)^{H} \mathbf{h}_{u}(j)\right\}\right|, \frac{2}{\beta_{k}}\left|\mathcal{J}\left\{\mathbf{h}_{u}(i)^{H} \mathbf{h}_{u}(j)\right\}\right|\right\} .
\end{aligned}
$$

For the case of $K=2$, all the error vectors $\mathbf{e}_{i j}$ having moduli lower than $T_{2}$ are used for FD calculation. Compared to $K=1$, we have to consider the added error vectors $\frac{1}{\sqrt{\beta_{k}}}\{ \pm 2 \pm$ $\left.2 \mathbf{j e}_{i}\right\}(i=1, \cdots, L)$ having $T_{2}=\sqrt{\frac{8}{\beta_{k}}}$, which belong to $\mathbb{D}_{1}$ and do not change the set $\mathbb{D}_{2}$. After eliminating all collinear elements, the set $\mathbb{D}_{1}$ of $K=2$ is reduced to $\frac{1}{\sqrt{\beta_{k}}}\left\{2 \mathbf{e}_{i}, \pm 2 \pm\right.$ $\left.2 \mathbf{j}_{i}\right\}, i=1, \cdots, L$. Moreover, since only the minimum distance is investigated, the set is further reduced to $\mathbb{D}_{1}=$ $\frac{1}{\sqrt{\beta_{k}}}\left\{2 \mathbf{e}_{i}\right\}, i=1, \cdots, L$, which is the same as that of $K=1$. Therefore, the setups of $K=1$ and $K=2$ will provide the same FD $d_{\min }\left(\mathbf{H}_{u}\right)$.

Moreover, for the case of $K=3$, besides the error vectors $\mathbf{e}_{i j}$ for $K=2$, the error vectors having $T_{3}=\sqrt{\frac{10}{\beta_{k}}}$ should be considered, which are given by $\frac{1}{\sqrt{\beta_{k}}}\left\{( \pm 3 \pm 1 \mathrm{j}) \mathbf{e}_{i}-( \pm 1 \pm 1 \mathrm{j}) \mathbf{e}_{j},( \pm 1 \pm 3 \mathrm{j}) \mathbf{e}_{i}-( \pm 1 \pm 1 \mathrm{j}) \mathbf{e}_{j}\right\}$, $i, j=1, \cdots, L, i \neq j$. For these added error vectors, we have $s_{a}^{H} s_{b} \in \frac{1}{\beta_{k}}\{ \pm 2 \pm 4 \mathrm{j}, \pm 4 \pm 2 \mathrm{j}\}$ and two legitimate combinations of the values of $\left|s_{a}\right|^{2}$ and $\left|s_{b}\right|^{2}$ as: (1) $\left|s_{a}\right|^{2}=\frac{2}{\beta_{k}},\left|s_{b}\right|^{2}=\frac{10}{\beta_{k}}$ and (2) $\left|s_{a}\right|^{2}=\frac{10}{\beta_{k}},\left|s_{b}\right|^{2}=\frac{2}{\beta_{k}}$. For each combination, similar 511 to the process of Eqs. (41)-(42), we can substitute the values 512 of $\left|s_{a}\right|^{2},\left|s_{b}\right|^{2}$ and $s_{a}^{H} s_{b}$ into Eq. (37) and get the simplified 513 optimized metric for $K=3$ as

$$
d_{\min , K=3}^{\text {joint }-E V M}=\min \left\{d_{\min , K=1}^{\text {joint }-E V M}, d_{\min ,(1)}^{\text {joint }-E V M}, d_{\min ,(2)}^{\text {joint }-E V M}\right\}
$$

where $d_{\min ,(1)}^{\mathrm{join}-E V M}$ and $d_{\min ,(2)}^{\mathrm{joint}-E V M}$ are the simplified ED for the 515 above-mentioned two combinations, given by Eq. (44), shown 516 at the bottom of the page.

4) The Proposed EVM-Based ED-TAS: Based on the sim- 518 plified versions of $d_{\mathrm{min}}^{\text {joint }-E V M}$ for $M$-PSK and $M$-QAM 519 schemes derived in Eqs. (41) and (43), the solution of our 520 EVM-based ED-TAS algorithm is given by

$$
\mathbf{H}_{\hat{u}}=\arg \max _{u \in\left\{1, \cdots, N_{U}\right\}}\left\{d_{\text {min }}^{\text {signal }}, d_{\text {min }}^{\text {spatial }}, d_{\text {min }}^{\text {joint }-E V M}\right\} .
$$

Note that similar to the proposed QRD-TAS, the terms 522 $\left\|\mathbf{h}_{u}(i)\right\|_{F}^{2},\left\|\mathbf{h}_{u}(j)\right\|_{F}^{2}$ and $\mathbf{h}_{u}(i){ }^{H} \mathbf{h}_{u}(j)$ in Eqs. (40)-(44) are 523 elements of the matrix $\mathbf{H}^{H} \mathbf{H}$. Then, we can find the solu- 524 tion of Eq. (45) by reusing these elements for different TAS 525 candidates $\mathbf{H}_{u}$.

Fig. 3 shows the probability that the error vectors having the 527 minimum norm do result in finding the optimal ED-TAS solu- 528 tion as a function of $K$. For example, we have a probability 529 of $97 \%$ for 16-QAM modulated SM for $K=1$ using $N_{t}=4,530$ $L=2$ and $N_{r}=2$. Moreover, it is observed from Fig. 3 that 531 this probability is also high for other QAM schemes; hence the 532 EVM-based ED-TAS can be readily used in diverse scenarios. 533 In general, for striking a flexible BER vs complexity tradeoff, 534 we can adjust the parameter $K$ to reduce the search space to a 535 subset of the error vectors that may yield the optimal ED-TAS 536 solution with a high probability.

Note that in [17] a PEP-based TAS (PEP-TAS) algorithm was 538 proposed, which was based on a different search set reduction. 539 The main differences of the proposed EVM-TAS and the PEP- 540 TAS of [17] are:

- The PEP-TAS is based on the assumption that a smaller 542 APM symbol amplitude leads to a smaller distance $d_{\text {min }}^{\text {joint }}, 543$ whereas based on our analysis it is highly likely that an 544 error vector with a small norm yields the distance $d_{\min }^{\text {joint }} . \quad 545$

- Moreover, in EVM-TAS, we propose to use the parameter 546 $K$ for striking a flexible tradeoff between the conflicting 547 factors of the computational complexity imposed and the 548 attainable BER.

Remark: Compared to the EVM-TAS, the PEP-TAS con- 550 siders only the error vectors generated by $M$-QAM symbols 551 having the minimum amplitude. It can be shown that the non- 552 linear error vectors of the PEP-TAS are the same as those of the 553

$$
\left\{\begin{array}{l}
d_{\min ,(1)}^{\text {joint-EVM }}=\min _{\substack{i, j=1, \cdots, L \\
i \neq j}} \frac{2}{\beta_{k}}\left\|\mathbf{h}_{u}(i)\right\|_{F}^{2}+\frac{10}{\beta_{k}}\left\|\mathbf{h}_{u}(j)\right\|_{F}^{2}-2 m_{M-Q A M}^{K=3} \\
d_{\min ,(2)}^{\text {joint } E V M}=\min _{\substack{i, j=1, \cdots, L \\
i \neq j}} \frac{10}{\beta_{k}}\left\|\mathbf{h}_{u}(i)\right\|_{F}^{2}+\frac{2}{\beta_{k}}\left\|\mathbf{h}_{u}(j)\right\|_{F}^{2}-2 m_{M-Q A M}^{K=3} \\
m_{M-Q A M}^{K=3}=\max \frac{1}{\beta_{k}}\left\{\left|2 \mathcal{R}\left\{\mathbf{h}_{u}(i)^{H} \mathbf{h}_{u}(j)\right\}\right|+\left|4 J\left\{\mathbf{h}_{u}(i)^{H} \mathbf{h}_{u}(j)\right\}\right|,\left|4 \mathcal{R}\left\{\mathbf{h}_{u}(i)^{H} \mathbf{h}_{u}(j)\right\}\right|+\left|2 \mathcal{J}\left\{\mathbf{h}_{u}(i){ }^{H} \mathbf{h}_{u}(j)\right\}\right|\right\}
\end{array}\right.
$$




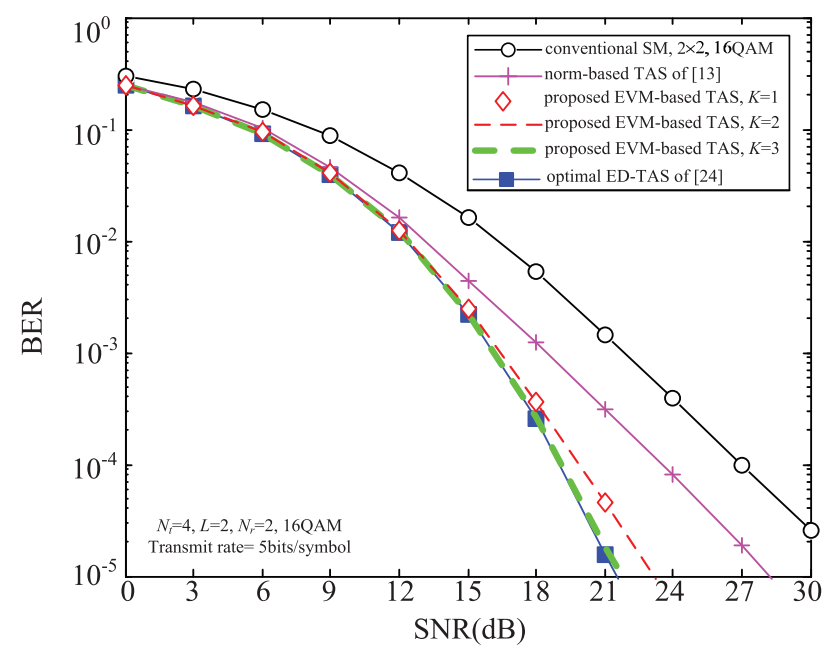

Fig. 4. BER performance comparison of the existing TAS algorithms and the proposed EVM-based TAS algorithm for $N_{t}=4, N_{r}=2,16 \mathrm{QAM}$ and $L=2$. The transmit rate is 5 bits/symbol.

EVM-TAS associated with $K=1$. Therefore, it can be viewed as a special case of EVM-TAS by setting $K=1$.

Fig. 4 shows our BER comparison for the existing TAS algorithms and the proposed EVM-TAS algorithm. The simulation parameters are the same as those of Fig. 2. Firstly, as proved in Section IV-B and observed in Fig. 3, the probability that the error vectors do indeed result in the optimal ED-TAS solution is the same for the cases of $K=1$ and $K=2$. Hence, they provide the same BER performance, as shown in Fig. 4. Furthermore, we observe in Fig. 3 that this probability is increased from 0.975 to 0.998 upon increasing $K$ from 1 to 3. As a result, in Fig. 4 the performance of the EVM-based ED-TAS associated with $K=3$ is improved compared to that scheme with $K=1$. Moreover, compared the results in Figs. 2 and 4, the EVM-based ED-TAS outperforms the SVD-based ED-TAS for $K=3$.

\section{JOINT TAS AND PA ALGORITHMS FOR SM}

Similar to the TAS technique, PA is another attractive link adaptation technique conceived for SM, which has been advocated in [7], [11], [28], [29]. The process of PA can be modeled by the PA matrix $\mathbf{P}$, which is given by

$$
\mathbf{P}=\operatorname{diag}\left\{p_{1}, \cdots, p_{q}, \cdots, p_{L}\right\},
$$

where $p_{q}$ controls the channel gain of the $q t h$ TA. Here, we let $\sum_{q=1}^{L} p_{q}^{2}=1$ for normalizing the transmit power. Based on our TAS algorithms, we propose a pair of combined algorithms for jointly considering the PA and TAS as follows:

\section{1) TAS\&PA}

- Step 1: Each $\left(N_{r} \times N_{t}\right)$ channel matrix $\mathbf{H}$ has $N_{U}=$ $\left(\begin{array}{c}N_{t} \\ L\end{array}\right)$ possible subchannel matrices $\mathbf{H}_{u}$, each of which corresponds to a specifically selected $\left(N_{r} \times\right.$ $L)$ MIMO channel. For each $\mathbf{H}_{u}$, we calculate the corresponding PA matrix $\mathbf{P}_{u}$ and its FD with the aid of the algorithm of [29].

- Step 2: The particular combinations of $\mathbf{H}_{u} \mathbf{P}_{u}(u=$ $\left.1, \cdots, N_{U}\right)$ constitute the legitimate TAS\&PA candidates. Let us interpret the matrices $\mathbf{H}_{u} \mathbf{P}_{u} 588$ ( $\left.u=1, \cdots, N_{U}\right)$ as being the equivalent channel 589 matrices of Section IV and select the specific can- 590 didate with the maximum free distance as the final 591 solution.

Since for each channel realization $\mathbf{H}$, there are $N_{U}$ pos- 593 sible PA matrices $\mathbf{P}_{u}\left(u=1, \cdots, N_{U}\right)$, we have a high 594 computational complexity if $N_{U}$ is high. Next, we intro- 595 duce a lower-complexity solution for this joint TAS and 596 PA algorithm.

2) Low-complexity TAS\&PA the proposed low-complexity QRD-based ED-TAS 600 or the EVM-based ED-TAS algorithm to select a 601 particular subset of TAs from the set of options, 602 which corresponds to $\mathbf{H}_{\hat{u}}$.

- Step 2: Calculate the power weights for the selected 604 TAs, which can be represented by the PA matrix $\mathbf{P}_{\hat{u}}$. 605 During this step, the low-complexity PA algorithm 606 of [29] can be invoked. In the simple TAS\&PA, the 607 PA matrix only has to be calculated once, hence the 608 associated complexity is low.

\section{Simulation Results}

In this section, we provide simulation results for further char- 611 acterizing the proposed QRD-based ED-TAS, EVM-based ED- 612 TAS and TAS\&PA schemes for transmission over frequency- 613 flat fading MIMO channels. For comparison, these performance 614 results are compared to various existing TAS-SM schemes of 615 [13], [21], [23], [25], to the classic TAS/maximal-ratio combin- 616 ing (TAS/MRC) schemes of [40], as well as to the TAS\&PA 617 aided V-BLAST of [32]. In our simulations, the single-stream 618 ML detector of [34], [35] is utilized.

\section{A. BER Comparisons of Different TAS Algorithms for SM}

In Fig. 5, we compare the BER performance of various TAS- 621 SM schemes for 4 bits/symbol associated with $N_{t}=8, L=4, \quad 622$ $N_{r}=4$ and QPSK. We also considered the conventional single- 623 RF based TAS/MRC arrangement of [40] as benchmarker. As 624 seen from Fig. 5, the proposed QRD-based ED-TAS outper- 625 forms the conventional SVD-based ED-TAS of [23], as also 626 formally shown in Fig. 2. Moreover, as expected, in Fig. 5627 the EVM-based TAS is capable of achieving the same per- 628 formance as the optimal ED-TAS of [21]. We also confirm 629 that our proposed EVM-based ED-TAS schemes outperform 630 the norm-based TAS of [13] and the QRD-based ED-TAS pro- 631 posed for PSK modulation. These results are consistent with the 632 analysis results in Section IV, where the EVM-based TAS has 633 considered all legitimate error vectors for simplifying $d_{\min }^{\text {joint }}$ in 634 Eq. (40), while the QRD-based ED-TAS may achieve uncorrect 635 estimation of $d_{\mathrm{min}}^{\text {joint }}$ due to the employment of lower bound of 636 Eq. (27).

Fig. 5 also shows that our new TAS-SM schemes outper- 638 form the TAS/MRC scheme of [40]. The main reason behind 639 the poorer performance of TAS/MRC is the employment of 640 a higher modulation order required for achieving the same 641 


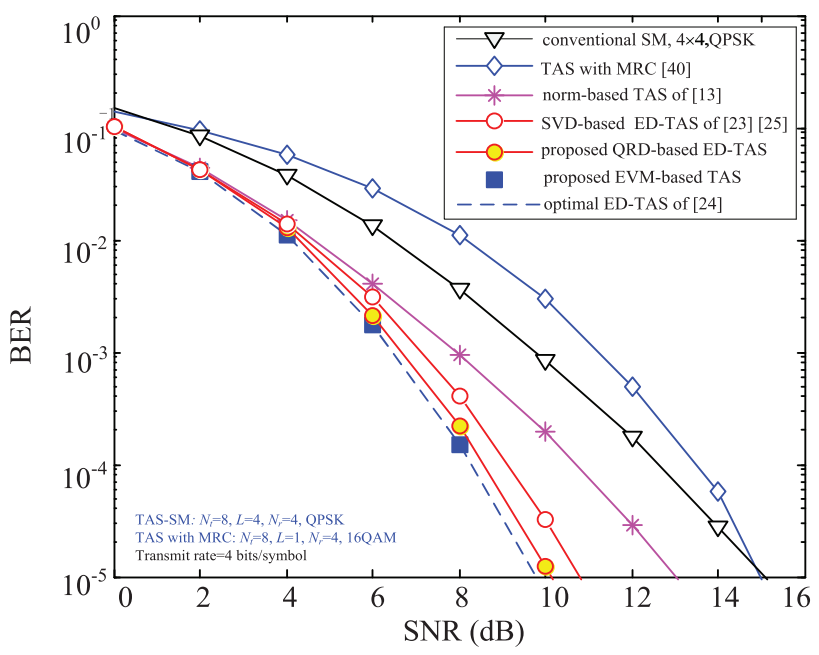

Fig. 5. BER comparison at $m=4$ bits/symbol for the proposed TAS-SM schemes, the existing TAS-SM schemes and the classic TAS/MRC scheme having $N_{t}=8$ and $L=4$.

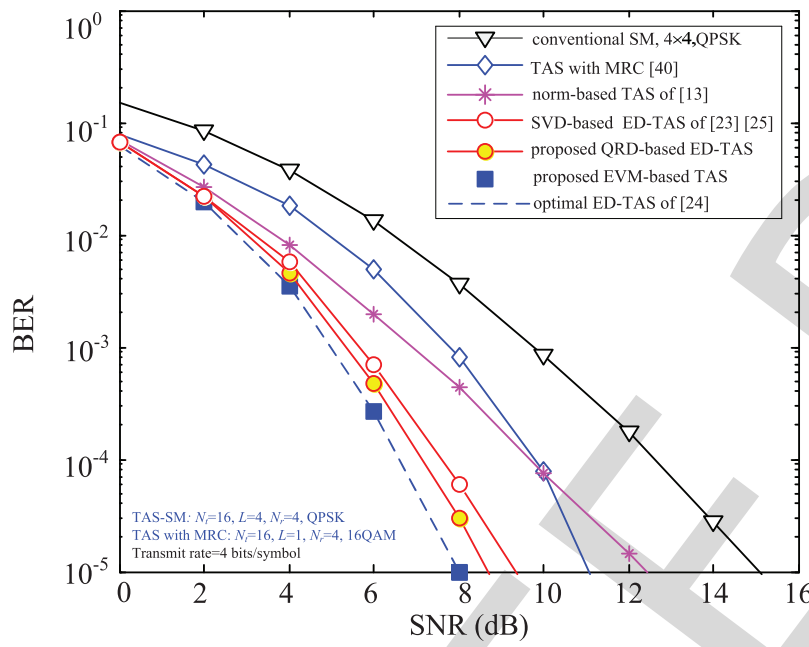

Fig. 6. BER comparison at $m=4$ bits/symbol for the proposed TAS-SM schemes, the existing TAS-SM schemes and the classic TAS/MRC scheme having $N_{t}=16$ and $L=4$.

throughput as our SM-based schemes. Note that this benefit depends on the particular MIMO setups. To be specific, as noted in [23], the TAS-SM and the TAS/MRC schemes exhibit different BER advantages for different system setups. However, similar to the results achieved in [23], our new TAS-SM schemes strike an attractive tradeoff between the complexity and the BER attained. The above-mentioned trends of these proposed TAS-SM schemes are also confirmed in Fig. 6, where the number $N_{t}$ of TAs increases from 8 to 16 .

In Fig. 7, a spatially correlated MIMO channel model characterized by $\mathbf{H}^{\text {corr }}=\mathbf{R}_{r}^{1 / 2} \mathbf{H} \mathbf{R}_{t}^{1 / 2}$ [24], [41] is considered for the proposed QRD-based ED-TAS and EVM-based TAS $(K=3)$ schemes, where $\mathbf{R}_{t}=\left[r_{i j}\right]_{N_{t} \times N_{t}}$ and $\mathbf{R}_{r}=\left[r_{i j}\right]_{N_{r} \times N_{r}}$ are the positive definite Hermitian matrices that specify the transmit and receive correlations, respectively. In Fig. 7, the components of $\mathbf{R}_{t}$ and $\mathbf{R}_{r}$ are calculated as $r_{i j}=r_{j i}^{*}=r^{j-i}$ for $i \leq j$, where $r$ is the correlation coefficient $(0 \leq r \leq 1)$. Here, the simulation parameters are the same as those of Figs. 2 and 4

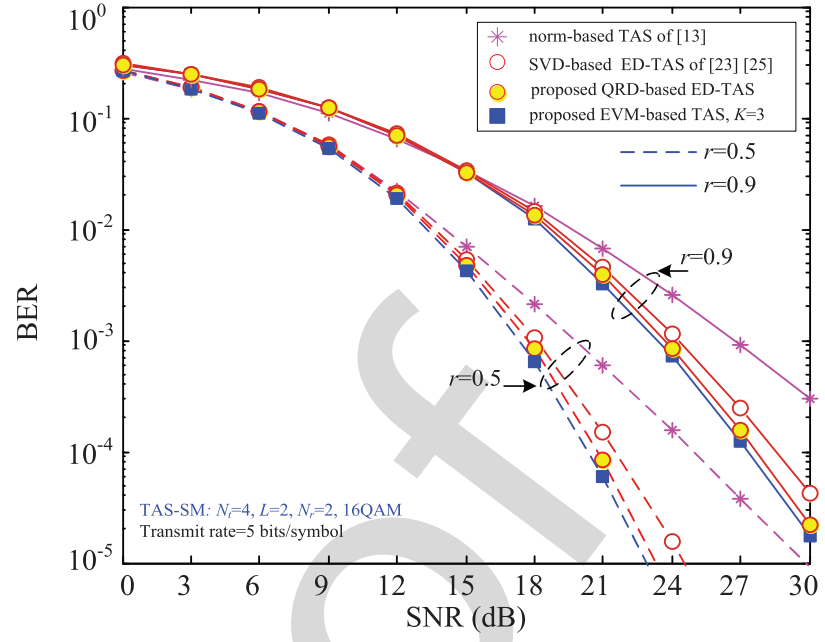

Fig. 7. BER comparison of different TAS algorithms for SM systems in correlated Rayleigh fading channels.

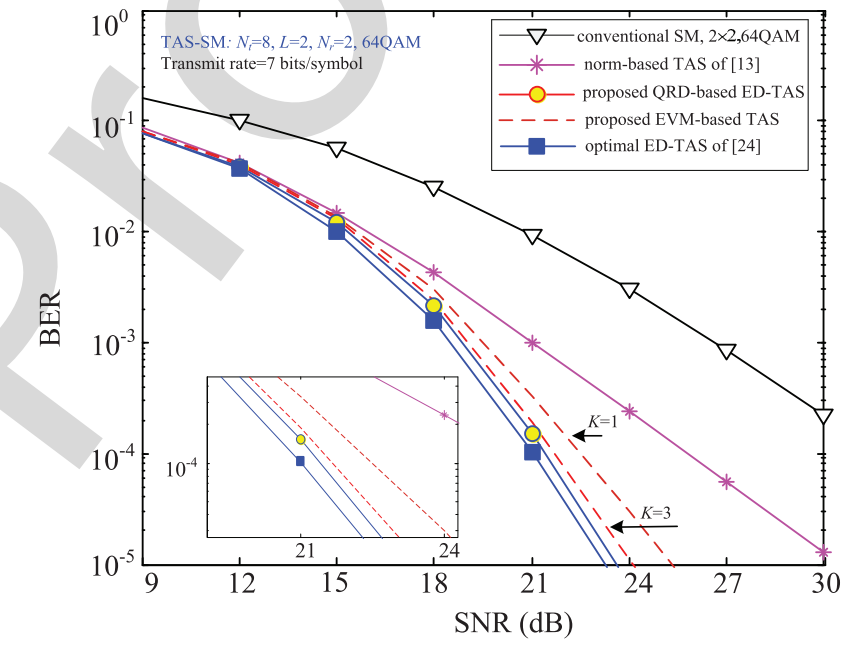

Fig. 8. BER comparison at $m=7$ bits/symbol for the proposed QRD-based ED-TAS and EVM-based ED-TAS with 64-QAM.

for 5 bits/symbol transmissions. We found that the BER curves 660 of the EVM-based TAS schemes and of the optimal ED-TAS 661 are almost overlapped (similar to the results seen in Fig. 4), 662 hence for clarity in Fig. 7 we simply provide the BER curves 663 for the EVM-based TAS schemes only. Compared to the BER 664 curves in Figs. 2 and 4 for the correlation coefficient $r=0$, we 665 observe in Fig. 7 that the BER performance of all schemes is 666 substantially degraded by these correlations. However, the pro- 667 posed schemes remain capable of operating efficiently for the 668 correlated channels.

In Fig. 8, we further compare the proposed QRD-based 670 ED-TAS scheme and the proposed EVM-based TAS schemes 671 for a higher modulation order, where the 64-QAM scheme is 672 employed. Observe in Fig. 8 that the proposed QRD-based 673 ED-TAS scheme outperforms the EVM-based TAS scheme in 674 conjunction with $K=1$ and the corresponding performance 675 gain is seen to be about $1 \mathrm{~dB}$. Similar to the results in Figs. 2676 and 4, the EVM-based TAS associated with $K=3$ provide 677 an improved BER compared to that scheme with $K=1$. At 678 


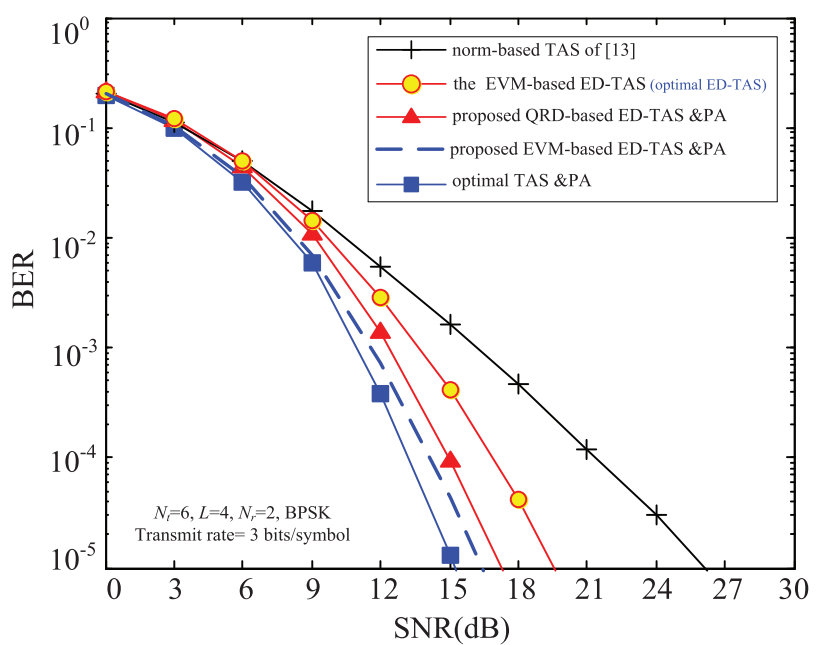

Fig. 9. BER performance comparison of the TAS algorithms and of the proposed TAS \&PA algorithms in SM systems, having the transmit rate of 3 bits/symbol.

$\mathrm{BER}=10^{-5}$, the performance gap between the proposed EVMbased TAS with $K=3$ and the proposed QRD-based ED-TAS becomes negligible.

The main conclusions observed from Figs. 2, 4 and 5-8 are: (1) the proposed EVM-based TAS and QRD-based ED-TAS schemes exhibit different BER advantages for different system setups; (2) the proposed QRD-based ED-TAS is preferred to the QAM-modulated SM schemes, since its complexity is independent of the modulation order; (3) The proposed EVMbased TAS is preferred to the PSK-modulated SM schemes, since it can achieve the performance of optimal ED-TAS at the reduced error vector set. (4) For the QAM-modulated SM schemes, the parameter $K$ of the proposed EVM-based TAS can be flexibly selected for striking a beneficial trade-off between the complexity imposed and the BER attained.

\section{B. BER Comparisons of TAS Algorithms and TAS \&PA} Algorithms for SM

In this subsection, we focus our attention on studying the BER performance of our TAS\&PA algorithms. Here, for the low-complexity TAS\&PA, the proposed QRD-based ED-TAS as well as the EVM-based ED-TAS algorithms are utilized and the corresponding algorithms are termed as the QRD-based ED-TAS \&PA and the EVM-based ED-TAS \&PA, respectively. Note that the EVM-based ED-TAS achieves the same performance as the optimal ED-TAS for the PSK-modulated SM schemes. The BER performances of other TAS algorithms are similar to the results seen in Figs. 2, 4 and 5-8. Hence, for clarity, when only pure TAS is considered, we simply provide the corresponding BER curves of the proposed EVMbased ED-TAS and of the conventional norm-based TAS as benchmarkers.

Fig. 9 compares the BER performance of the proposed TAS\&PA arrangement to that of other SM-based schemes. In Fig. 9, the parameter setup is $N_{t}=6, L=4, N_{r}=2$ and $M=2$. It becomes clear from Fig. 9 that the TAS\&PA algorithms advocated outperform both the EVM-based ED-TAS and

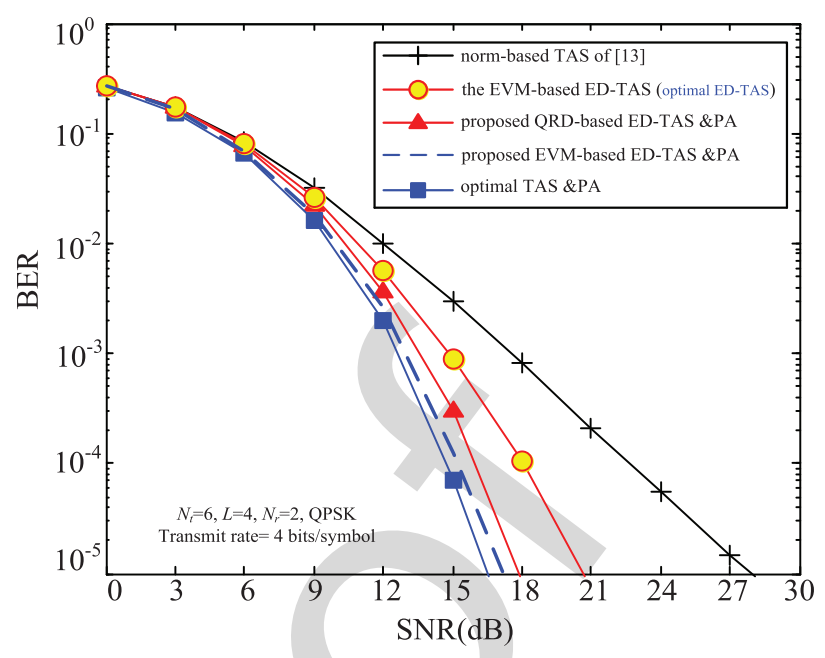

Fig. 10. BER performance comparison of the TAS algorithms and of the proposed TAS \&PA algorithms in SM systems, having the transmit rate of 4 bits/symbol.

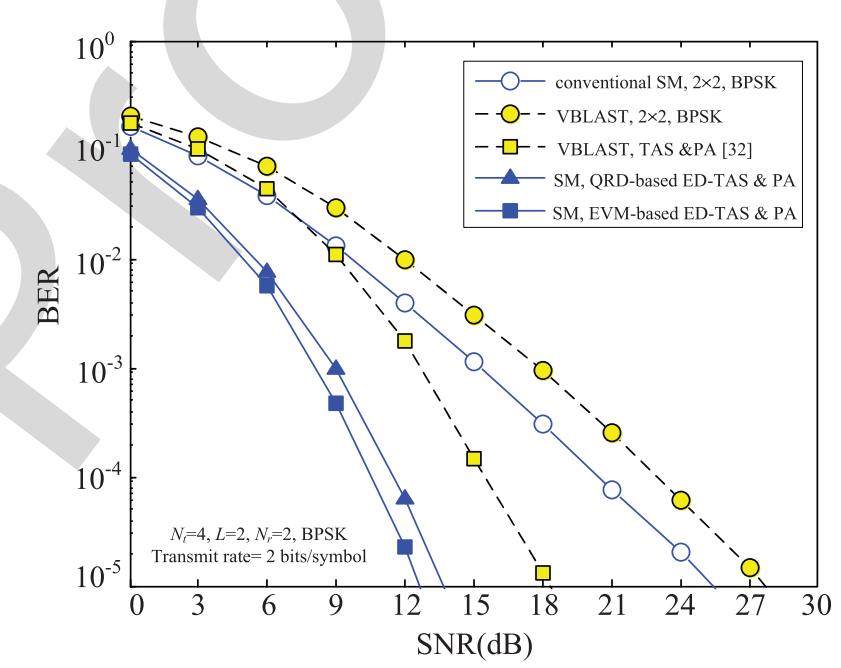

Fig. 11. BER performance comparison of the proposed TAS \&PA algorithms in SM systems and the conventional identical-throughput TAS\&TA algorithm in V-BLAST systems, where the throughput is 2 bits/symbol $\left(N_{t}=4, N_{r}=2\right.$, $L=2$ ).

the norm-based ED-TAS. At a BER of $10^{-5}$, the exhaustive- 715 search based optimal TAS\&PA provides $9.5 \mathrm{~dB}$ and $4 \mathrm{~dB}$ SNR 716 gains over the norm-based ED-TAS and over the EVM-based 717 ED-TAS, respectively. Moreover, the low-complexity QRD- 718 based ED-TAS \&PA provides about 4 dB SNR gain over the 719 EVM-based TAS operating without PA. 720

Fig. 9 also shows that the EVM-based ED-TAS \&PA outper- 721 forms the QRD-based ED-TAS\&PA and is capable of achieving 722 almost the same BER performance as the optimal TAS\&PA. 723 The performance advantages of our schemes are attained as 724 a result of exploiting all the benefits of MIMO channels. The 725 above-mentioned trends of these TAS\&PA algorithms recorded 726 for SM are also visible in Fig. 10, where a SM system using 727 $N_{t}=6, L=4, N_{r}=2$ and QPSK modulation is considered. 728

In Fig. 11, the BPSK-modulated V-BLAST scheme and its 729 TAS\&PA-aided counterpart [32] associated with zero-forcing 730 successive interference cancellation (ZF-SIC) are compared to 731 
TABLE II

COMPLEXITY COMPARISON OF DIFFERENT TAS-SM ALGORITHMS IN DIVERSE CONFIGURATIONS

\begin{tabular}{|c|c|c|c|}
\hline TAS algorithm & $\begin{array}{c}\text { Configuration 1 } \\
\left(N_{t}=4, N_{r}=2\right. \\
L=2,16 \mathrm{QAM})\end{array}$ & $\begin{array}{r}\text { Configuration } 2 \\
\left(N_{t}=8, N_{r}=4\right. \\
L=4, \text { QPSK })\end{array}$ & $\begin{array}{r}\text { Configuration } 3 \\
\left(N_{t}=8, N_{r}=2\right. \\
L=2,64-\mathrm{QAM})\end{array}$ \\
\hline Exhaustive ED-TAS [13] & 13824 & 8512 & 1032192 \\
\hline Maximum-norm based TAS [21] & 12 & 56 & 24 \\
\hline Conventional QRD-based ED-TAS [24] & 2060 & 6029 & 38253 \\
\hline SVD-based ED-TAS [25] & 102 & 588 & 444 \\
\hline Proposed QRD-based ED-TAS & 82 & 596 & 340 \\
\hline Proposed EVM-based ED-TAS & $\begin{array}{l}84, K=1 \\
180, K=3\end{array}$ & 756 & $\begin{array}{l}360, K=1 \\
808, K=3\end{array}$ \\
\hline Exhaustive TAS\&PA & 4626 & 46340 & 256788 \\
\hline Proposed QRD-based ED-TAS\&PA & 853 & 1004 & 9511 \\
\hline Proposed EVM-based ED-TAS\&PA & $\begin{array}{l}855, K=1 \\
951, K=3\end{array}$ & 1164 & $\begin{array}{c}9531, K=1 \\
9979, K=3\end{array}$ \\
\hline
\end{tabular}

our TAS\&PA based schemes. For maintaining an identicalthroughput, in Fig. 11 we let $N_{t}=4, N_{r}=2, L=2$ and use BPSK for all schemes. Observe in Fig. 11 that our TAS\&PA based SM schemes outperform the TAS\&PA aided V-BLAST schemes by about 5-6 dB SNR at the BER of $10^{-5}$.

\section{Complexity Comparison}

Table I shows the complexity comparison of various TAS algorithms conceived for SM, where the total number of floating point operations is considered. The Appendix provides the details of our computational complexity evaluations for the proposed TAS algorithms list in Table I. The complexity estimation of the existing TAS algorithms can be found in [15], [23] and [24]. Moreover, our complexity analysis is similar to that of [23] and [24].

Explicitly, in Table II, the quantified complexity of different TAS algorithms for some specific configurations are provided. As shown in Table I, the proposed QRD-based ED-TAS has a similar complexity order to that of the low-complexity SVDbased ED-TAS of [23], while exhibiting a lower complexity compared to the conventional QRD-based ED-TAS of [24]. For example, the proposed QRD-based ED-TAS imposes an approximately 168 times and 25 times lower complexity than the exhaustive ED-TAS and the conventional QRD-based EDTAS for configuration 1 . This is due to the fact that it is capable of avoiding the high-complexity QRD operation by directly computing the bound parameters of Eq. (27). Moreover, as shown in Tables I-II and Figs. 4-8, the EVM-based ED-TAS advocated is capable of striking a flexible BER vs complexity trade-off by employing the parameter $K$ for diverse $M$-QAM schemes. Furthermore, the proposed low-complexity TAS\&PA schemes impose a lower complexity than the exhaustive-search based TAS\&PA and only impose a slightly increased complexity compared to the proposed EVM-based TAS and QRD-based TAS schemes. By considering the BER vs complexity results of Tables I-II and Figs. 9-11, the proposed low-complexity TAS\&PA is seen to provide an improved BER performance at a modest complexity cost.

\section{CONCLUSIONS}

In this paper, we have investigated TAS algorithms conceived for SM systems. Firstly, a pair of low-complexity
ED-TAS algorithms, namely the QRD-based ED-TAS and the 772 EVM-based ED-TAS, were proposed. The theoretical analysis 773 and simulation results indicated that the QRD-based ED-TAS 774 exhibits a better BER performance compared with the conven- 775 tional SVD-based ED-TAS, while the EVM-based ED-TAS is 776 capable of striking a flexible BER vs complexity trade-off. To 777 further improve the attainable performance, the proposed TAS 778 algorithms were amalgamated with PA. A pair of beneficial 779 joint TAS-PA algorithms were proposed and our simulation 780 results demonstrated that they outperform both the pure TAS 781 algorithms and the TAS\&PA algorithm designed for spatial 782 multiplexing systems.

\section{APPENDIX}

Computational complexity of the proposed TAS algorithms 785 designed for SM systems.

\section{A. The Proposed QRD-Based ED-TAS}

As detailed in Section IV-A, the calculation of the QRD- 788 based bound of Eq. (27) only depends on the elements of 789 the matrix $\mathbf{H}^{H} \mathbf{H}$, which incurs a complexity in the order 790 of $\operatorname{comp}\left(\mathbf{H}^{H} \mathbf{H}\right)=2 N_{t}^{2} N_{r}-N_{t}^{2}$. Then, we can calculate the 791 values of $\tilde{R}_{k, k}\left(\boldsymbol{\Pi}_{m}\right),(m=1,2, k=1,2)$ in Eqs. (30)-(33) 792 by reusing these elements for the different TAS candi- 793 dates $\mathbf{H}_{u}$. Specifically, the calculation of $\sqrt{\left\|\mathbf{h}_{u}(j)\right\|_{F}^{2}}, j=794$ $1, \cdots, N_{t}$ for estimating $\tilde{R}_{1,1}\left(\Pi_{m}\right), m=1,2$ in Eqs. (30) and 795 (32) requires $N_{t}$ flops. Moreover, to calculate the values of 796 $\tilde{R}_{2,2}\left(\boldsymbol{\Pi}_{m}\right), m=1,2$ in Eqs. (31) and (33), we have to con- 797 sider $\left(\begin{array}{c}N_{t} \\ 2\end{array}\right)$ possible combinations $(i, j)$ for computing the value 798 of $\sqrt{\frac{\left\|\mathbf{h}_{u}(i)\right\|_{F}^{2}+\left\|\mathbf{h}_{u}(j)\right\|_{F}^{2}-2 \mathcal{R}\left\{\mathbf{h}_{u}(i)^{H} \mathbf{h}_{u}(j)\right\}}{\left\|\mathbf{h}_{u}(j)\right\|_{F}^{2}}}$. For each combination, 799 the complexity imposed is 5 flops. Hence, the complexity 800 of computing $\tilde{R}_{2,2}\left(\boldsymbol{\Pi}_{m}\right), m=1,2$ is $5\left(\begin{array}{c}N_{t} \\ 2\end{array}\right)$ flops. The overall 801 complexity of the proposed QRD-based ED-TAS is

$$
\begin{aligned}
C_{\mathrm{PQRD}} & =2 N_{t}^{2} N_{r}-N_{t}^{2}+N_{t}+5\left(\begin{array}{c}
N_{t} \\
2
\end{array}\right) \\
& =2 N_{t}^{2} N_{r}+\frac{3}{2} N_{t}\left(N_{t}-1\right) .
\end{aligned}
$$

Note that based on Eq. (28), $d_{\text {min }}^{\text {Modulus }}, d_{\min }^{\mathrm{APM}}$ and $d_{\min }^{\text {all }}$ are 803 constants for a specific APM scheme and the calculation of 804 $d_{\text {min }}^{\text {signal }}$ and $d_{\text {min }}^{\text {spatial }}$ can also exploit the common elements, such 805 as $\left\|\mathbf{h}_{u}(i)\right\|_{F}^{2}+\left\|\mathbf{h}_{u}(j)\right\|_{F}^{2}-2 \mathcal{R}\left\{\mathbf{h}_{u}(i)^{H} \mathbf{h}_{u}(j)\right\},\left\|\mathbf{h}_{u}(i)\right\|_{F}^{2}$, in the 806 
807 calculation of the bound of $d_{\min }^{\text {joint }}$, as shown in Eqs. (15) and 808

(16). Hence, the complexity imposed can be deemed negligible.

\section{B. The Proposed EVM-Based ED-TAS}

Similar to the proposed QRD-based ED-TAS, the computational complexity of EVM-based ED-TAS is also dominated by computing $d_{\min }^{\text {joint }}$. Specifically, we also first have to evaluate the elements $\left\|\mathbf{h}_{u}(i)\right\|_{F}^{2},\left\|\mathbf{h}_{u}(j)\right\|_{F}^{2}$ and $\mathbf{h}_{u}(i)^{H} \mathbf{h}_{u}(j)$, which incurs a complexity of $2 N_{t}^{2} N_{r}-N_{t}^{2}$ flops. Then, for $M$-PSK, the simplified version of $d_{\min }^{\text {joint }}$ is given in Eq. (40), which has to consider $\left(\begin{array}{c}N_{t} \\ 2\end{array}\right)$ legitimate TA combination $(i, j)$. For each combination $(i, j)$, the computation of the term $m_{M-\mathrm{PSK}}\left(\mathbf{H}_{u}\right)$ of Eq. (39) has to consider $\left(\frac{M}{4}+1\right)$ possible $\theta_{n}$ values. For each $\theta_{n}$, the complexity of evaluating $\left|\mathcal{R}\left\{\mathbf{h}_{u}(i){ }^{H} \mathbf{h}_{u}(j)\right\} \cos \theta_{n}-\mathcal{J}\left\{\mathbf{h}_{u}(i){ }^{H} \mathbf{h}_{u}(j)\right\} \sin \theta_{n}\right|$ is 4 flops. Moreover, for a specific $m_{M-\operatorname{PSK}}\left(\mathbf{H}_{u}\right)$ and a fixed combination $(i, j)$, the computation of $\left\|\mathbf{h}_{u}(i)\right\|_{F}^{2}+\left\|\mathbf{h}_{u}(j)\right\|_{F}^{2}-$ $2 m_{M-\mathrm{PSK}}\left(\mathbf{H}_{u}\right)$ in Eq. (40) requires 3 flops. Hence, the overall complexity of the $M$-PSK modulated EVM-based ED-TAS is

$$
\begin{aligned}
C_{\mathrm{EVM}} & =2 N_{t}^{2} N_{r}-N_{t}^{2}+\left(\begin{array}{c}
N_{t} \\
2
\end{array}\right)\left\{4\left(\frac{M}{4}+1\right)+3\right\} \\
& =2 N_{t}^{2} N_{r}-N_{t}^{2}+\frac{1}{2} N_{t}\left(N_{t}-1\right)(M+7) .
\end{aligned}
$$

For the $M$-QAM scheme, this complexity depends on the parameter $K$. Specifically, the simplified versions of $d_{\mathrm{min}}^{\text {joint }}$ are different for different values of $K$. In general, for a given $K$, we first characterize all possible combinations of $\left|s_{a}\right|^{2}$ and $\left|s_{b}\right|^{2}$ by using the method of Section IV-B. Let us assume that the number of these combinations is $G$. For each combination, we can simplify Eq. (37) similar to the process of Eqs. (43)-(44), which corresponds to $G$ simplified equations and each requires 15 flops, as shown in Eq. (37). Since $\left(\begin{array}{c}N_{t} \\ 2\end{array}\right)$ legitimate TA combinations $(i, j)$ should be considered in Eq. (37), we arrive at a complexity of $15 G\left(\begin{array}{c}N_{t} \\ 2\end{array}\right)$ for all possible combinations. Overall, the complexity of the EVM-based TAS for $M$-QAM modulated $\mathrm{SM}$ is

$$
C_{\mathrm{EVM}}=2 N_{t}^{2} N_{r}-N_{t}^{2}+15 G\left(\begin{array}{c}
N_{t} \\
2
\end{array}\right) .
$$

Note that the complexity of Eq. (49) is an approximate result, which can be further refined based on the specific simplified version of $d_{\min }^{\text {joint }}$. For example, based on Eqs. (41) and (43) derived for $K=1$ and $K=3$, similar to the complexity analysis of $M$-PSK, the computational complexity orders of the EVM-based TAS for $K=1$ and $K=3$ are

$$
C_{\mathrm{EVM}-\mathrm{TAS}}=2 N_{t}^{2} N_{r}-N_{t}^{2}+6\left(\begin{array}{c}
N_{t} \\
2
\end{array}\right),
$$

and

$$
C_{\mathrm{EVM}-\mathrm{TAS}}=2 N_{t}^{2} N_{r}-N_{t}^{2}+22\left(\begin{array}{c}
N_{t} \\
2
\end{array}\right)
$$

\section{The Proposed PA \& TAS}

The exhaustive-search based TAS\&PA algorithm has to calculate all legitimate PA matrix candidates. According to Section
$\mathrm{V}$, there are $N_{U}=\left(\begin{array}{c}N_{t} \\ L\end{array}\right)$ legitimate PA matrix candidates $\mathbf{P}_{u}(u=848$ $\left.1, \cdots, N_{U}\right)$, which can be obtained by using the method pro- 849 posed in [29]. The complexity of computing each PA matrix is 850 $C_{\mathrm{PA}}$ (Eq. (22) in [29]) flops. Hence, the associated complexity 851 of the exhaustive-search based TAS\&PA algorithm is $N_{U} C_{\mathrm{PA}} 852$ flops. By contrast, the low-complexity TAS\&PA algorithm first 853 selects the optimal TA subset and then calculates the PA matrix 854 for the selected set. Hence, the associated complexity order of 855 the low-complexity TAS\&PA algorithm is $C_{\mathrm{TAS}}+C_{\mathrm{PA}}$ flops, 856 where $C_{\text {TAS }}$ is the complexity of the TAS algorithm employed, 857 i. e. $C_{\mathrm{EVM}}$ or $C_{\mathrm{PQRD}}$.

\section{REFERENCES}

[1] R. Y. Mesleh, H. Haas, S. Sinanovic, C. W. Ahn, and S. Yun, "Spatial 860 modulation," IEEE Trans. Veh. Technol., vol. 57, no. 4, pp. 2228-2241, 861 Jul. 2008.

[2] M. Di Renzo, H. Haas, A. Ghrayeb, S. Sugiura, and L. Hanzo, "Spatial 863 modulation for generalized MIMO: Challenges, opportunities and imple- 864 mentation," Proc. IEEE, vol. 102, no. 1, pp. 56-103, Jan. 2014.

[3] S. Sugiura, S. Chen, and L. Hanzo, "A universal space-time architec- 866 ture for multiple-antenna aided systems," IEEE Commun. Surveys Tuts., 867 vol. 14, no. 2, pp. 401-420, May 2012.

[4] M. Di Renzo, H. Haas, and P. M. Grant, "Spatial modulation for multiple- 869 antenna wireless systems: A survey," IEEE Commun. Mag., vol. 49, 870 no. 12, pp. 182-191, Dec. 2011.

[5] A. Stavridis, S. Sinanovic, M. Di Renzo, and H. Haas, "Energy evaluation 872 of spatial modulation at a multi-antenna base station," in Proc. IEEE Veh. 873 Technol. Conf., Barcelona, Spain, Sep. 2013, pp. 1-5.

[6] M. Di Renzo and H. Haas, "Bit error probability of SM-MIMO over 875 generalized fading channels," IEEE Trans. Veh. Technol., vol. 61, no. 3, 876 pp. 1124-1144, Mar. 2012.

[7] P. Yang, M. Di Renzo, Y. Xiao, S. Li, and L. Hanzo, "Design guidelines 878 for spatial modulation," IEEE Commun. Surveys Tuts., vol. 17, no. 1, 879 pp. 6-26, Mar. 2015.

[8] S. Sugiura and L. Hanzo, "On the joint optimization of dispersion matri- 881 ces and constellations for near-capacity irregular precoded space-time 882 shift keying," IEEE Wireless Commun., vol. 12, no. 1, pp. 380-387, Jan. 883 2013.

[9] P. Yang, Y. Xiao, Y. Yi, and S. Li, "Adaptive spatial modulation for wire- 885 less MIMO transmission systems," IEEE Commun. Lett., vol. 15, no. 6, 886 pp. 602-604, Jun. 2011.

[10] P. Yang, Y. Xiao, L. Li, Q. Tang, Y. Yu, and S. Li, "Link adaptation for 888 spatial modulation with limited feedback," IEEE Trans. Veh. Technol., 889 vol. 61 , no. 8 , pp. $3808-3813$, Oct. 2012.

[11] M. Di Renzo and H. Haas, "Improving the performance of space shift 891 keying (SSK) modulation via opportunistic power allocation," IEEE 892 Commun. Lett., vol. 14, no. 6, pp. 500-502, Jun. 2010.

[12] S. Sanayei and A. Nosratinia, "Antenna selection in MIMO systems," 894 IEEE Commun. Mag., vol. 42, no. 10, pp. 68-73, Oct. 2004.

[13] W. H. Chung, C. Y. Hung, Q. Zhang, and M. Gao, "Multi-antenna selec- 896 tion using space shift keying in MIMO systems," in Proc. IEEE Veh. 897 Technol. Conf., May 2012, pp. 1-5.

[14] B. Kumbhani and R. S. Kshetrimayum, "Outage probability analysis 899 of spatial modulation systems with antenna selection," Electron. Lett., 900 vol. 50, no. 2, pp. 125-126, 2014.

[15] S. Sugiura, S. Chen, and L. Hanzo, "Coherent and differential space- 902 time shift keying: A dispersion matrix approach," IEEE Trans. Commun., 903 vol. 58, no. 11, pp. 3219-3230, Nov. 2010.

[16] J Wang et al., "Closed-loop spatial modulation with antenna selection," 905 in Proc. IEEE 11th Int. Conf. Signal Process., Oct. 2012, pp. 1291-1294. 906

[17] Z. Zhou, N. Ge, and X. Lin, "Reduced-complexity antenna selection 907 schemes in spatial modulation," IEEE Commun. Lett., vol. 18, no. 1, 908 pp. 14-17, Jun. 2014.

[18] M. Maleki, H. R. Bahrami, and A. Alizadeh, "Adaptive antenna sub- 910 set selection and constellation breakdown for spatial modulation," IEEE 911 Commun. Lett., vol. 18, no. 9, pp. 1649-1652, Sep. 2014.

[19] X. Wu, M. D. Renzo, and H. Haas, "Direct transmit antenna selection 913 for transmit optimized spatial modulation," in Proc. IEEE Veh. Technol. 914 Conf. (VTC'13-Fall), Las Vegas, NV, USA, Sep. 2013, pp. 1-5. 915

[20] X. Wu, M. Di Renzo, and M. Haas, "Adaptive selection of antennas 916 for optimum transmission in spatial modulation," IEEE Trans. Wireless 917 Commun., vol. 14, no. 7, pp. 3630-3641, Jul. 2015. 
[21] R. Rajashekar, K. V. S. Hari, and L. Hanzo, "Antenna selection in spatial modulation systems," IEEE Commun. Lett., vol. 17, no. 3, pp. 521-524, Mar. 2013.

[22] N. Pillay and H. Xu, "Comments on "Antenna selection in spatial modulation systems"," IEEE Commun. Lett., vol. 17, no. 9, pp. 1681-1683, Sep. 2013.

[23] K. Ntontin, M. Di Renzo, A. Perez-Neira, and C. Verikoukis, "A lowcomplexity method for antenna selection in spatial modulation systems," IEEE Commun. Lett., vol. 17, no. 12, pp. 2312-2315, Aug. 2013.

[24] N. Wang, W. Liu, H. Men, M. Jin, and H. Xu. "Further complexity reduction using rotational symmetry for EDAS in spatial modulation," IEEE Commun. Lett., vol. 18, no. 10, pp. 1835-1838, Oct. 2014.

[25] J. Zheng and J. Chen, "Further complexity reduction for antenna selection in spatial modulation systems," IEEE Commun. Lett., vol. 19, no. 6, pp. 937-940, Jun. 2015.

[26] R. Rajashekar, K. V. S. Hari, and L. Hanzo, "Quantifying the transmit diversity order of Euclidean distance based antenna selection in spatial modulation," IEEE Commun. Lett., vol. 22, no. 9, pp. 1434-1437, Sep. 2015.

[27] N. Pillay and X. Hongjun, "Low-complexity transmit antenna selection schemes for spatial modulation," IET Commun., vol. 9, no. 2, pp. 239248, Jan. 2015.

[28] P. Yang, Y. Xiao, B. Zhang, S. Li, M. El-Hajjar, and L. Hanzo, "Power allocation aided spatial modulation for limited-feedback MIMO systems," IEEE Trans. Veh. Technol., vol. 64, no. 5, pp. 2198-2204, May 2015.

[29] P. Yang, Y. Xiao, S. Li, and L. Hanzo, "A low-complexity power allocation algorithm for multiple-input multiple-output spatial modulation systems," IEEE Trans. Veh. Technol., vol. 65, no. 3, pp. 1819-1825, Mar. 2016.

[30] M. Di Renzo and H. Haas, "Improving the performance of space shift keying (SSK) modulation via opportunistic power allocation," IEEE Commun. Lett., vol. 14, no. 6, pp. 500-502, Jun. 2010.

[31] Y. Xiao, Q. Tang, L. Gong, P. Yang, and Z. Yang, "Power scaling for spatial modulation with limited feedback," Int. J. Antennas Propag., vol. 20135 p., 2013.

[32] Z. Shi and H. Leib, "Transmit antenna selection V-BLAST systems with power allocation," IEEE Trans. Veh. Technol., vol. 57, no. 4, pp. 2293 2304, Jul. 2008

[33] R. Rajashekar, K. V. S. Hari, and L. Hanzo, "Reduced-complexity ML detection and capacity-optimized training for spatial modulation," IEEE Trans. Commun., vol. 62, no. 1, pp. 112-125, Jan. 2014.

[34] S. Sugiura, C. Xu, S. X. Ng, and L. Hanzo, "Reduced complexity coherent versus non-coherent QAM-aided space time shift keying," IEEE Trans. Commun., vol. 59, no. 11, pp. 3090-3101, Nov. 2011.

[35] A. Younis, S. Sinanovic, M. Di Renzo, R. Y. Mesleh, and H. Haas, "Generalised sphere decoding for spatial modulation," IEEE Trans. Commun., vol. 61, no. 7, pp. 2805-2815, Jul. 2013.

[36] A. Goldsmith, Wireless Communications. Cambridge, U.K.: Cambridge Univ. Press, 2005.

[37] G. Golub and C. F. van Loan, Matrix Computations, 3 rd ed. Baltimore, MD, USA: The Johns Hopkins Univ. Press, 1996.

[38] J. K. Zhang, A. Kavcic, and K. M. Wong, "Equal-diagonal QR decomposition and its application to precoder design for successive-cancellation detection," IEEE Trans. Inf. Theory, vol. 51, no. 1, pp. 154-172, Jan. 2005.

[39] J. Lee, S. Y. Jung, and D. Park, "Simplified maximum-likelihood precoder selection for spatial multiplexing systems," IEEE Trans. Veh. Technol., vol. 59, no. 9, pp. 4628-4634, Nov. 2010.

[40] Z. Chen, J. Yuan, and B. Vucetic, "Analysis of transmit antenna selection/maximal-ratio combining in Rayleigh fading channels," IEEE Trans. Veh. Techol., vol. 54, no. 4, pp. 1312-1321, Jul. 2005.

[41] V. Raghavan, J. J. Choi, and D. J. Love, "Design guidelines for limited feedback in the spatially correlated broadcast channel," IEEE Trans. Commun, vol. 63, no. 7, pp. 2524-2540, Jul. 2015.

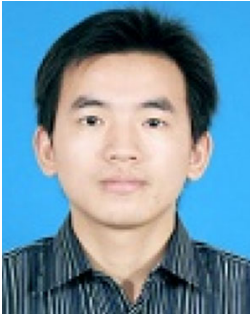

Ping Yang (SM'xx) received the Ph.D. degree from the University of Electronic Science and Technology of China (UESTC), Chengdu, China, in 2013. From September 2012 to September 2013, he was a Visiting Student at the School of Electronics and Computer Science, University of Southampton, Southampton, U.K. Since May 2014, he has been a Research Fellow in EEE of NTU, Singapore. Also, he is an Assistant Professor with UESTC. His research interests include MIMO/OFDM, machine learning, life science, and communication signal processing.

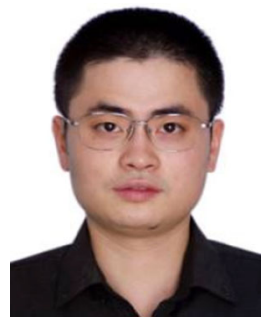

Yue Xiao (M'xx) received the Ph.D. degree in 995 communication and information systems from the 996 University of Electronic Science and Technology of 997 China, Chengdu, China, in 2007. He is now a Full 998 Professor with the University of Electronic Science 999 and Technology of China. He has authored more than 1000 30 international journals and been involved in several 1001 projects in Chinese Beyond 3G Communication R\&D 1002 Program. His research interests include wireless and 1003 mobile communications.

1004

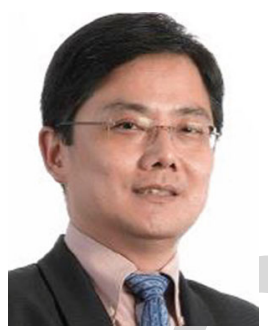

Yong Liang Guan (M'xx) received the Ph.D. degree 1005 from the Imperial College of Science, Technology, 1006 and Medicine, University of London, London, U.K., 1007 in 1997, and the B.Eng. degree (with first class 1008 Hons.) from the National University of Singapore, 1009 Singapore, in 1991. He is now an Associate 1010 Professor with the School of Electrical and Electronic 1011 Engineering, Nanyang Technological University, 1012 Singapore. His research interests include modulation, 1013 coding and signal processing for communication, 1014 information security and storage systems.

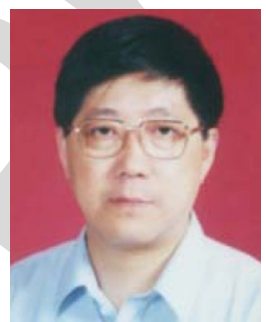

Shaoqian Li (F'16) received the B.S.E. degree in 1016 communication technology from Northwest Institute 1017 of Telecommunication (Xidian University), Xi'an, 1018 China, in 1982, and the M.S.E. degree in communica- 1019 tion system from the University of Electronic Science 1020 and Technology of China (UESTC), Chengdu, China, 1021 in 1984. He is a Professor, Ph.D. Supervisor, 1022 and the Director of the National Key Laboratory 1023 of Communication, UESTC, and member of the 1024 National High Technology R\&D Program (863 1025 Program) Communications Group. His research inter- 1026 ests includes wireless communication theory, anti-interference technology for 1027 wireless communications, spread-spectrum and frequency-hopping technology, 1028 and mobile and personal communications.

1029

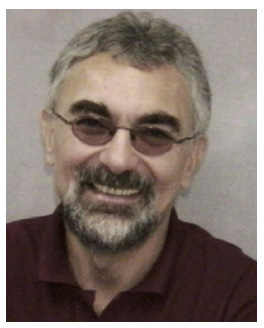

Lajos Hanzo (F'xx) received the degree in elec- 1030 tronics, in 1976, the doctorate degree, in 1983, 1031 and the D.Sc. degree. During his 37-year career in 1032 telecommunications, he has held various research 1033 and academic posts in Hungary, Germany, and the 1034 U.K. Since 1986, he has been with the School of 1035 Electronics and Computer Science, University of 1036 Southampton, Southampton, U.K., where he holds 1037 the chair in telecommunications. He has successfully 1038 supervised over $80 \mathrm{Ph} . \mathrm{D}$. students, coauthored 201039 John Wiley/IEEE Press books on mobile radio com- 1040 munications totalling in excess of 10000 pages, published 1400+ research 1041 entries at IEEE Xplore, acted both as TPC and the General Chair of the IEEE 1042 conferences, presented keynote lectures and has been awarded a number of 1043 distinctions. Currently, he is directing a 100-strong academic research team, 1044 working on a range of research projects in the field of wireless multimedia 1045 communications sponsored by industry, the Engineering and Physical Sciences 1046 Research Council (EPSRC) U.K., the European Research Council's Advanced 1047 Fellow Grant and the Royal Society's Wolfson Research Merit Award. He is an 1048 enthusiastic supporter of industrial and academic liaison and offers a range of 1049 industrial courses. He is also a Governor of the IEEE VTS. From 2008 to 2012, 1050 he was the Editor-in-Chief of the IEEE Press and a Chaired Professor also at 1051 Tsinghua University, Beijing, China. His research is funded by the European 1052 Research Council's Senior Research Fellow Grant. He has more than 190001053 citations. In 2009, he was received the honorary doctorate "Doctor Honoris 1054 Causa" by the Technical University of Budapest, Budapest, Hungary. He is the 1055 fellow of the REng, IET, and EURASIP. 


\section{QUERIES}

Q1: Please be advised that per instructions from the Communications Society this proof was formatted in Times Roman font and therefore some of the fonts will appear different from the fonts in your originally submitted manuscript. For instance, the math calligraphy font may appear different due to usage of the usepackage[mathcal]euscript. We are no longer permitted to use Computer Modern fonts.

Q2: Note that if you require corrections/changes to tables or figures, you must supply the revised files, as these items are not edited for you.

Q3: Please provide IEEE membership details of authors "Ping Yang, Yue Xiao, Yong Liang Guan, and Lajos Hanzo." 


\title{
Transmit Antenna Selection for Multiple-Input Multiple-Output Spatial Modulation Systems
}

\author{
Ping Yang, Senior Member, IEEE, Yue Xiao, Member, IEEE, Yong Liang Guan, Member, IEEE, \\ Shaoqian Li, Fellow, IEEE, and Lajos Hanzo, Fellow, IEEE
}

\begin{abstract}
The benefits of transmit antenna selection (TAS) invoked for spatial modulation (SM) aided multiple-input multiple-output (MIMO) systems are investigated. Specifically, we commence with a brief review of the existing TAS algorithms and focus on the recently proposed Euclidean distance-based TAS (ED-TAS) schemes due to their high diversity gain. Then, a pair of novel ED-TAS algorithms, termed as the improved QR decomposition (QRD)-based TAS (QRD-TAS) and the error-vector magnitude-based TAS (EVM-TAS) are proposed, which exhibit an attractive system performance at low complexity. Moreover, the proposed ED-TAS algorithms are amalgamated with the low-complexity yet efficient power allocation (PA) technique, termed as TAS-PA, for the sake of further improving the system's performance. Our simulation results show that the proposed TAS-PA algorithms achieve signal-to-noise ratio (SNR) gains of up to $9 \mathrm{~dB}$ over the conventional TAS algorithms and up to $6 \mathrm{~dB}$ over the TAS-PA algorithm designed for spatial multiplexing systems.
\end{abstract}

Index Terms-Antenna selection, MIMO, power allocation, spatial modulation, link adaptation.

\section{INTRODUCTION}

$\mathbf{S}$ PATIAL modulation (SM) and its variants constitute a class of promising low-complexity and low-cost multipleinput multiple-output (MIMO) transmission techniques [1]-[5]. However, the conventional SM schemes only achieve receiverdiversity, but no transmit diversity [6]. To circumvent this impediment, recently some SM solutions have been proposed [7]-[11] on how to glean a beneficial transmit-diversity gain both with the aid of open-loop as well as closed-loop transmitsymbol design techniques.

As an attractive closed-loop regime, transmit antenna selection (TAS) constitutes a promising technique of providing a

Manuscript received October 10, 2015; revised February 22, 2016; accepted March 24, 2016. This work was supported of the National Science Foundation of China under Grant 61501095, in part by the National High-Tech R\&D Program of China ("863" Project under Grant 2014AA01A707), and in part by the European Research Council's Advanced Fellow Grant. The associate editor coordinating the review of this paper and approving it for publication was V. Raghavan.

P. Yang, Y. Xiao, and S. Li are with the National Key Laboratory of Science and Technology on Communications, University of Electronic Science and Technology of China, Sichuan 611731, China (e-mail: yplxw@163.com; xiaoyue@uestc.edu.cn; 1sq@uestc.edu.cn).

Y. L. Guan is with the School of Electrical and Electronic Engineering, Nanyang Technological University, Singapore (e-mail: eylguan@ntu.edu.sg).

L. Hanzo is with the School of Electronics and Computer Science, University of Southampton, Southampton SO17 1BJ, U.K. (e-mail: 1h@ecs.soton.ac.uk).

Color versions of one or more of the figures in this paper are available online at http://ieeexplore.iee.org.

Digital Object Identifier 10.1109/TCOMM.2016.2547900 high diversity potential as offered by the classic MIMO architectures. TAS has been lavishly researched in the context of spatial multiplexing systems [12]. As a new MIMO technique, SM can also be beneficially combined with TAS. Recently, several TAS algorithms have been conceived for the class of SM-MIMO systems with the goal of enhancing either its bit error rate (BER) or its capacity [13]-[20]. In [13], a normbased TAS algorithm was proposed for providing diversity gain. In [14], a closed-form expression of the SM scheme's outage probability was derived for norm-based TAS. In [16], a twostage TAS-based SM scheme was proposed for overcoming the specific constraint of SM, namely that the number of transmit antennas has to be a power of two. In [17], a novel TAS criterion was proposed for circumventing the detrimental effects of antenna correlation. In [18], the joint design of TAS and constellation breakdown was investigated and a graph-based search algorithm was proposed for reducing the search complexity imposed. In [19], a low-complexity TAS algorithm based on circle packing was proposed for a transmitter-optimized spatial modulation (TOSM) system, which trades off the spatial constellation size against the amplitude and phase modulation (APM) constellation size for improving the system's average bit error probability (ABEP). The adaptive TAS algorithm conceived for TOSM was further developed in [20], where a low-complexity two-stage optimization was proposed for selecting the best transmission mode.

More recently, the research of TAS-aided SM has been focused on the optimization of the Euclidean Distance (ED) of the received constellation points, since they achieve a high diversity gain at a moderate complexity compared to other TAS criteria [21]-[24]. Specifically, in [21] and [22] the EDbased TAS algorithm (ED-TAS) was compared to the signalto-noise ratio (SNR)-optimized and capacity-optimized algorithms, and a low-complexity realization of ED-TAS, termed as the QR decomposition-based TAS (QRD-TAS) was proposed. The QRD-TAS algorithm constructs an ED-element matrix and exploits the QRD of the resultant matrix for reducing the imposed complexity. Moreover, in [24], the authors exploited the rotational symmetry of the APM adopted for the sake of reducing the complexity of QRD-TAS. Compared to directly optimizing the ED, in [23], Ntontin et al. proposed a low-complexity singular value decomposition-based TAS (SVD-TAS) algorithm for maximizing the lower bound of the ED. In [25], the complexity of SVD-TAS was reduced through an alternative computation of the singular value. In [26], the transmit diversity order of ED-TAS was quantified. In [27], the authors proposed several low-complexity TAS schemes relying 
on exploiting the channel's amplitude, the antenna correlation, the ED between transmit vectors and their combinations for selecting the optimal TA subset for the sake of improving the system's reliability. However, as shown in [21]-[27], the QRD-TAS achieves an attractive BER performance at the cost of adopting high-complexity QRD operations, while the low-complexity SVD-TAS may suffer some performance loss.

On the other hand, power allocation (PA) is another promising link adaptation technique for MIMO systems. Recently, PA has been extended to SM systems [28]-[31]. For example, in [28], an adaptive PA algorithm based on maximizing the minimum ED was proposed, which is capable of improving the system's BER performance, while retaining all the single-RF benefits of SM. Subsequently, this attractive PA algorithm was further simplified in [29]. However, to the best of our knowledge, the potential benefits of TAS intrinsically amalgamated with PA have not been investigated in SM-MIMO systems.

Against this background, the contributions of this paper are:

1) We investigate the benefits of ED-TAS and propose a pair of novel ED-TAS schemes for SM-MIMO systems. In these schemes, we first classify the legitimate EDs into three specific subsets and then invoke a carefully designed upper bound as well as a set-reduction method for the most dominant set imposing a high complexity.

2) Specifically, we propose an improved QRD-TAS, where a tighter QRD-based lower bound of the ED is derived to replace the SVD-based bound of [23]. A low-complexity method is proposed for directly calculating the bound parameters, in order to avoid the high-complexity QRD or SVD operations of [21]-[24]. More importantly, compared to the conventional SVD-TAS of [25], the achieved QRD-based tighter bound can achieve a better BER performance.

3) Moreover, for striking a flexible tradeoff in terms of the BER attained and the complexity imposed, we propose an error-vector magnitude based TAS (EVM-TAS), which exploits the error vector selection probability to shrink the search space. The relevant optimization metrics of EVM-TAS are also derived for different PSK and QAM schemes.

4) Finally, we intrinsically amalgamate the proposed EDTAS with the recently conceived PA technique of [29] for fully exploiting the MIMO channel's resources. A pair of different joint TAS-PA algorithms are conceived, which provide beneficial gains over both the conventional TAS algorithms and over the TAS-PA techniques designed for spatial multiplexing systems [32].

The organization of the paper is as follows. Section II introduces the system model of TAS-based SM, while Section III reviews the family of existing TAS algorithms designed for SM. In Section IV, we introduce the proposed QRD-TAS and EVM-TAS algorithms. In Section V, the joint design of the EDTAS and PA algorithms is proposed. Then, we carry out their complexity analysis. Our simulation results and performance comparisons are presented in Section VI. Finally, Section VII concludes the paper.

Notation: $(\cdot)^{*},(\cdot)^{T}$ and $(\cdot)^{H}$ denote conjugate, transpose, and Hermitian transpose, respectively. Furthermore, $\|\cdot\|_{F}$ stands for the Frobenius norm. $\mathbf{I}_{b}$ denotes a $(b \times b)$-element identity matrix and the operator $\operatorname{diag}\{\cdot\}$ is the diagonal operator. $\mathcal{R}\{\mathbf{x}\}$ and $\mathcal{J}\{\mathbf{x}\}$ represent the real and imaginary parts of $\mathbf{x}$ respectively.

142 143Q1 144

\section{SYSTEM MODEL}

Consider a SM system having $N_{t}$ transmit and $N_{r} 147$ receive antennas, as depicted in Fig. 1. The frequency- 148 flat quasi-static fading MIMO channel is represented 149 by $\mathbf{H}=\left[\mathbf{h}(1), \mathbf{h}(2), \cdots, \mathbf{h}\left(N_{t}\right)\right] \sim \mathcal{C N}\left(0, \mathbf{I}_{N_{r} \times N_{t}}\right)$, where 150 $\mathbf{h}(1), \mathbf{h}(2), \cdots, \mathbf{h}\left(N_{t}\right)$ are the column vectors corresponding 151 to each transmit antenna (TA) in $\mathbf{H}$. The receiver first selects 152 $L$ TAs according to a specific selection criterion. Then, the 153 receiver sends this information to the transmitter via a feedback 154 link. As shown in [23], let $U_{u}$ denote the $u t h$ legitimate TA 155 subset, where we have

$$
\begin{aligned}
& U_{1}=\{1,2, \cdots, L\}, \\
& U_{2}=\{1,2, \cdots, L-1, L+1\}, \\
& \vdots \\
& U_{N_{U}}=\left\{N_{t}-L+1, \cdots, N_{t}\right\} .
\end{aligned}
$$

In Eq. (1), there are $N_{U}=\left(\begin{array}{c}N_{t} \\ L\end{array}\right)$ possible TA subsets, each of 157 which corresponds to an $\left(N_{r} \times L\right)$-element MIMO channel. As 158 shown in Fig. $1, \mathbf{b}=\left[b_{1}, \ldots, b_{L}\right]$ is the transmit bit vector in 159 each time slot, which contains $m=\log _{2}(L M)$ bits, where $M$ is 160 the size of the APM constellation. In SM, the input vector $\mathbf{b}$ is 161 partitioned into two sub-vectors of $\log _{2}(L)$ and $\log _{2}(M)$ bits, 162 denoted as $\mathbf{b}_{1}$ and $\mathbf{b}_{2}$, respectively. The bits in $\mathbf{b}_{1}$ are used for 163 selecting a unique TA index $q$ for activation, while the bits of 164 $\mathbf{b}_{2}$ are mapped to a Gray-coded APM symbol $s_{l}^{q} \in \mathbb{S}$. Then, the 165 SM symbol $\mathbf{x} \in \mathbb{C}^{L \times 1}$ is formulated as

$$
\mathbf{x}=s_{l}^{q} \mathbf{e}_{q}=\left[0, \cdots, s_{l}^{q}, \cdots, 0\right]^{T},
$$

where $\mathbf{e}_{q}(1 \leq q \leq L)$ is selected from the $L$-dimensional basis 167 vectors (as exemplified by $\mathbf{e}_{1}=[1,0, \cdots, 0]^{T}$ ). In the sce- 168 nario that $U_{u}$ is selected, the signal observed at the $N_{r}$ receive 169 antennas is given by

$$
\mathbf{y}=\mathbf{H}_{u} \mathbf{x}+\mathbf{n}
$$

where $\mathbf{H}_{u}$ is the $\left(N_{r} \times L\right)$-element TAS matrix correspond- 171 ing to the selected TA set $U_{u}$, and $\mathbf{n}$ is the $\left(N_{r} \times 1\right)$-element 172 noise vector. The elements of the noise vector $\mathbf{n}$ are complex 173 Gaussian random variables obeying $\mathrm{eN}\left(0, N_{0}\right)$.

The receiver performs maximum-likelihood (ML) detection 175 over all legitimate $\mathrm{SM}$ symbols $\mathbf{x} \in \mathbb{C}^{L \times 1}$ to obtain

$$
\hat{\mathbf{x}}=\arg \min _{\mathbf{x} \in \mathbb{X}}\left\|\mathbf{y}-\mathbf{H}_{u} \mathbf{x}\right\|_{F}^{2}=\arg \min _{\mathbf{x} \in \mathbb{X}}\left\|\mathbf{y}-\mathbf{h}_{u}(q) s_{l}^{q}\right\|_{F}^{2},
$$

where $\mathbb{X}$ is the set of all legitimate transmit symbols and $\mathbf{h}_{u}(q) \quad 177$ is the qth column of the equivalent channel matrix $\mathbf{H}_{u}$. The 178 complexity of the single-stream ML detection of Eq. (4) is low, 179 since a single TA is activated during any time slot [34], [35]. 


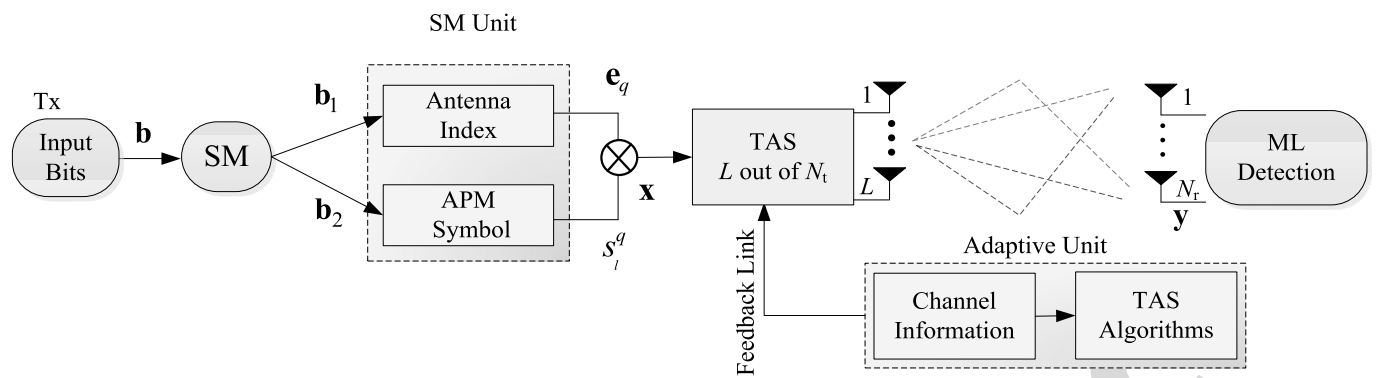

Fig. 1. The system model of the TAS-based SM system.

\section{Conventional TAS Algorithms}

This section offers a brief state-of-the-art review of the existing TAS algorithms proposed for SM systems.

\section{A. The Maximum-Capacity and The Maximum-Norm Based TAS Algorithms}

The capacity $C_{u}$ of the SM-aided MIMO system depends on the classic transmitted signal $s_{l}^{q}$ and the TA index signal $\mathbf{e}_{q}$. As shown in [21], [33], the capacity $C_{\mathrm{s}}$ relying on the signal $s_{l}^{q}$ and the channel $\mathbf{H}_{u}$ is lower bounded by

$$
\alpha=\frac{1}{L} \sum_{i=1}^{L} \log _{2}\left(1+\rho\left\|\mathbf{h}_{u}(i)\right\|_{F}^{2}\right) \leq C_{\mathrm{s}},
$$

where $\mathbf{h}_{u}(i)$ is the ith column of $\mathbf{H}_{u}$ and $\rho$ is the average SNR at the receiver. Moreover, the capacity $C_{\mathrm{TA}}$ relying on the signal $\mathbf{e}_{q}$ is bounded by $C_{\mathrm{TA}} \leq \log _{2}(L)$ [33]. It is proved in [33] that the total capacity $C_{u}=C_{\mathrm{TA}}+C_{\mathrm{s}}$ is bounded by

$$
\alpha \leq C_{u} \leq \alpha+\log _{2}(L),
$$

Based on the bound of Eq. (6), a maximum-capacity based TAS algorithm was formulated in [21] as

$$
\mathbf{H}_{\hat{u}}=\arg \max _{u \in\left\{1, \cdots, N_{U}\right\}} \alpha .
$$

Based on Eq. (5), the optimization objective $\alpha$ of Eq. (7) is maximized by selecting the $L$ TAs associated with the largest channel norms out of the $N_{t}$ TAs, which is equivalent to the maximum-norm based TAS [13] given by

$$
\mathbf{H}_{\hat{u}}=\arg \max _{u \in\left\{1, \cdots, N_{U}\right\}}\left\|\mathbf{H}_{u}\right\|_{F}^{2} .
$$

\section{B. The Exhaustive Max- $d_{\min }$ Based ED-TAS}

In order to improve the BER performance of SM, the free distance (FD) $d_{\min }$ was optimized in [21]. For a given channel $\mathbf{H}_{u}$, its FD can be formulated as

$$
\begin{aligned}
d_{\min }\left(\mathbf{H}_{u}\right) & =\min _{\substack{\mathbf{x}_{i}, \mathbf{x}_{j} \in \mathbb{X} \\
\mathbf{x}_{i} \neq \mathbf{x}_{j}}}\left\|\mathbf{H}_{u}\left(\mathbf{x}_{i}-\mathbf{x}_{j}\right)\right\|_{F}^{2} \\
& =\min _{\mathbf{e}_{i j} \in \mathbb{E}}\left\|\mathbf{H}_{u} \mathbf{e}_{i j}\right\|_{F}^{2}=\min _{\mathbf{e}_{i j} \in \mathbb{E}} \mathbf{e}_{i j}^{H} \mathbf{H}_{u}^{H} \mathbf{H}_{u} \mathbf{e}_{i j},
\end{aligned}
$$

where we have the error vector $\mathbf{e}_{i j}=\mathbf{x}_{i}-\mathbf{x}_{j}, \mathbf{x}_{i}, \mathbf{x}_{j} \in \mathbb{X}$. In 204 [21], the $\max -d_{\min }$ aided ED-TAS algorithm is defined as 205

$$
\mathbf{H}_{\hat{u}}=\arg \max _{u \in\left\{1, \cdots, N_{U}\right\}} d_{\min }\left(\mathbf{H}_{u}\right) .
$$

The optimum solution obeying the objective function of 206 Eq. (10) can be found by an exhaustive search over all possi- 207 ble $\left(\begin{array}{c}N_{t} \\ L\end{array}\right)$ candidate channel matrices and all the different error 208 vectors, which imposes a complexity order of $\mathcal{O}\left(N_{t}^{2} M^{2}\right)$. This 209 results in an excessive complexity, when high data rates are 210 required.

\section{The Conventional QRD-Based ED-TAS}

In order to reduce the complexity of the exhaustive ED-TAS 213 of Eq. (10), in [21] an ED-TAS based on an equivalent decision 214 metric $\mathbf{D}(u)$ was formulated as:

$$
\mathbf{H}_{\hat{u}}=\arg \max _{u \in\left\{1, \cdots, N_{U}\right\}}\{\min [\mathbf{D}(u)]\},
$$

where $\mathbf{D}(u)$ is an $(L \times L)$-element sub-matrix of an upper tri- 216 angular $\left(N_{t} \times N_{t}\right)$-element matrix $\mathbf{D}$ obtained by deleting the 217 specific rows and columns that are absent in $u$, while $\min [\mathbf{D}(u)] 218$ is the minimum non-zero value of $\mathbf{D}(u)$. Here, the $(i, j)-t h 219$ element of $\mathbf{D}$ can be expressed as

$$
\begin{aligned}
\mathbf{D}_{i j} & =\min _{s_{1}, s_{2} \in \mathbb{S}}\left\|\mathbf{H}\left(s_{1} \mathbf{e}_{i}-s_{2} \mathbf{e}_{j}\right)\right\|_{F}^{2} \\
& =\min _{s_{1}, s_{2} \in \mathbb{S}}\left\|\mathbf{h}(i) s_{1}-\mathbf{h}(j) s_{2}\right\|_{F}^{2},
\end{aligned}
$$

where $s_{1}$ and $s_{2}$ are $M$-ary APM constellation points, 221 while $\mathbf{h}(i)$ and $\mathbf{h}(j)$ are the ith and $j$ th columns of 222 H. Provided that we have $i=j$ in Eq. (12), the corre- 223 sponding element becomes $\mathbf{D}_{i i}=\min _{s_{1}, s_{2} \in \mathbb{S}}\left(\|\mathbf{h}(i)\|_{F}^{2}\left|s_{1}-s_{2}\right|^{2}\right)=224$ $d_{\min }^{\mathrm{APM}}\|\mathbf{h}(i)\|_{F}^{2}$, where $d_{\min }^{\mathrm{APM}}$ is the minimum distance of the 225 APM constellation. For the case of $i \neq j, \mathbf{D}_{i j}$ is re-formulated 226 in the real-valued representation of the QRD as

$$
\mathbf{D}_{i j}=\min _{\substack{s_{1 I}, s_{2 I} \in \mathcal{R}\{\mathbb{S}\}, s_{1} Q, s_{2} Q \in \mathcal{I}\{\}}}\left\|\mathbf{R}\left[s_{1 I}, s_{1 Q},-s_{2 I},-s_{2 Q}\right]^{T}\right\|_{F}^{2},
$$

where we have $s_{n I}=\mathcal{R}\left\{s_{n}\right\}$ and $s_{n} Q=\mathcal{J}\left\{s_{n}\right\}$ for $n=1,2,228$ while $\mathbf{R}$ is a $(4 \times 4)$-element upper triangular matrix created 229 by the QRD of the resultant channel matrix [21]. As shown in 230 [21], the complexity order of this QRD-TAS is $\mathcal{O}\left(N_{t}^{2} M\right)$, which 231 
increases only linearly with the modulation order $M$. In [22] and [24], both the modulus and the symbol set symmetry of the APM constellations were exploited for further reducing the complexity of this algorithm.

\section{The Conventional SVD-Based ED-TAS}

Although the QRD-based ED-TAS of Eq. (13) is capable of finding the optimal solution, its complexity imposed is a function of the modulation order $M$. Moreover, the high-complexity QRD has to be applied to the $\left(2 N_{r} \times 4\right)$-element channel matrices [21], [22], [24]. Hence, the complexity of this TAS remains high. This problem was circumvented in [23], where the ED was classified into three categories as follows

$$
d_{\min }\left(\mathbf{H}_{u}\right)=\min \left\{d_{\min }^{\text {signal }}, d_{\min }^{\text {spatial }}, d_{\min }^{\text {joint }}\right\}
$$

where we have

$$
\begin{aligned}
d_{\text {min }}^{\text {signal }} & =\min _{\substack{i=1, \cdots, L \\
\text { mPM }}}\left\|\mathbf{h}_{u}(i)\right\|_{F}^{2} \min _{s_{a} \neq s_{b} \in \mathbb{S}}\left|s_{a}-s_{b}\right|^{2} \\
& =d_{\min }^{\mathrm{APM}} \mathbf{h}_{u}(i) \|_{F}^{2}, \\
d_{\min }^{\text {spatial }} & =\min _{\substack{i, j=1, \cdots, L \\
i \neq j}}\left\|\mathbf{h}_{u}(i)-\mathbf{h}_{u}(j)\right\|_{F}^{2} \min _{s_{l} \in \mathbb{S}}\left|s_{l}\right|^{2} \\
& =d_{\min }^{\text {Modulus }} \min _{\substack{i, j=1, \cdots, L \\
i \neq j}}\left\|\mathbf{h}_{u}(i)-\mathbf{h}_{u}(j)\right\|_{F}^{2}, \\
d_{\min }^{\text {joint }} & =\min _{\substack{i, j=1, \cdots, L, i \neq j \\
s_{a}, s_{b}, \in \mathbb{S}, a \neq b}}\left\|\mathbf{h}_{u}(i) s_{a}-\mathbf{h}_{u}(j) s_{b}\right\|_{F}^{2} .
\end{aligned}
$$

In Eq. (16), the term $d_{\min }^{\text {Modulus }}=\min _{s_{l} \in \mathbb{S}}\left|s_{l}\right|^{2}$ is the minimum squared modulus value of the APM constellation. Since the calculations of $d_{\mathrm{min}}^{\text {signal }}$ and $d_{\mathrm{min}}^{\text {spatial }}$ in Eqs. (15) and (16) do not depend on the size of APM constellation and the corresponding complexity is low, the complexity of computing the FD of Eq. (14) is dominated by the computation of $d_{\mathrm{min}}^{\text {joint }}$ in Eq. (17). To reduce this complexity, in [23] the Rayleigh-Ritz theorem was utilized for driving a lower bound of $d_{\min }^{\text {joint }}$ as

$$
\begin{aligned}
d_{\min }^{\text {joint }} & =\min _{\substack{i, j=1, \cdots, L, i \neq j \\
s_{a}, s_{b} \in \mathbb{S}, a \neq b}}\left\|\left[\mathbf{h}_{u}(i),-\mathbf{h}_{u}(j)\right]\left[s_{a}, s_{b}\right]^{T}\right\|_{F}^{2} \\
\geq & d_{\min }^{\mathrm{SVD}-\text { bound }} \\
& =\min _{i, j=1, \cdots, L, i \neq j} \lambda_{\min }^{2}\left(\mathbf{H}_{u, i j}\right) \min _{s_{a}, s_{b} \in \mathbb{S}}\left\|\left[s_{a}, s_{b}\right]^{T}\right\|_{F}^{2} \\
& =\min _{i, j=1, \cdots, L, i \neq j} \lambda_{\min }^{2}\left(\mathbf{H}_{u, i j}\right) d_{\min }^{\text {all }}
\end{aligned}
$$

where we have $d_{\min }^{\text {all }}=\min _{s_{a}, s_{b} \in \mathbb{S}}\left\|\left[s_{a}, s_{b}\right]^{T}\right\|_{F}^{2}$ and $\mathbf{H}_{u, i j}=$ $\left[\mathbf{h}_{u}(i),-\mathbf{h}_{u}(j)\right]$ is an $\left(N_{r} \times 2\right)$-element matrix. Here, $\lambda_{\min }^{2}\left(\mathbf{H}_{u, i j}\right)$ is the minimum squared singular value of the submatrix $\mathbf{H}_{u, i j}$. Upon exploiting Eq. (18), the distance $d_{\min }\left(\mathbf{H}_{u}\right)$ of Eq. (14) is bounded by

$$
d_{\min }^{\mathrm{SVD}}\left(\mathbf{H}_{u}\right)=\min \left\{d_{\min }^{\mathrm{signal}}, d_{\min }^{\text {spatial }}, d_{\min }^{\mathrm{SVD}-\text { bound }}\right\} .
$$

Based on Eq. (19), the SVD-TAS algorithm is given by

$$
\mathbf{H}_{\hat{u}}=\arg \max _{u \in\left\{1, \cdots, N_{U}\right\}} d_{\min }^{\mathrm{SVD}}\left(\mathbf{H}_{u}\right) .
$$

Compared to the conventional QRD-based TAS, this bound- 259 aided algorithm has the following advantages:

- Using the SVD-based bound of Eq. (18), the calcula- 261 tion of the distance $d_{\min }^{\text {joint }}$ is independent of the APM 262 modulation order;

- Moreover, the SVD operation of Eq. (18) is performed 264 on the smaller channel matrices of size $\left(N_{r} \times 2\right)$ com- 265 pared to the QRD-based ED-TAS, which is performed on 266 $\left(2 N_{r} \times 4\right)$-element matrices. In [25], the complexity of 267 SVD-TAS [23] was further reduced through an alternative 268 computation of the singular value.

\section{The Proposed LOW-COMPLEXITY ED-TAS}

As shown in subsection III, the conventional QRD-based 271 ED-TAS is capable of achieving the optimal BER, but it 272 imposes high complexity. In contrast, the SVD-based ED-TAS 273 imposes a lower complexity at the cost of a BER performance 274 degradation, because the derived bound may be loose and the 275 corresponding TAS results may be suboptimal. 276

To circumvent this problem, in this section, a pair of ED-TAS 277 algorithms are proposed. Specifically, an improved QRD-TAS 278 is proposed, where a tighter QRD-based lower bound of the 279 ED is found for replacing the SVD-based bound of [23], while 280 the sparse nature ${ }^{1}$ of the error vectors of SM is exploited to 281 avoid the full-dimensional QRD operation. Then, for striking 282 a further flexible BER vs complexity tradeoff, we propose an 283 EVM-based ED-TAS algorithm, which exploits the error vector 284 selection probability to shrink the search space.

\section{A. The Proposed QRD-Based ED-TAS}

1) The QRD-Based Bounds: To evaluate the value of $d_{\min }^{\text {joint }} 287$ more accurately, in this paper, we apply the QRD-based bound 288 to replace the SVD-bound of Eq. (18). Specifically, the sub- 289 matrix $\mathbf{H}_{u, i j}$ of Eq. (18) is first subjected to the QRD [38], 290 yielding $\mathbf{H}_{u, i j}=\tilde{\mathbf{Q}} \tilde{\mathbf{R}}$, where $\tilde{\mathbf{Q}}$ is an $\left(N_{r} \times 2\right)$ column-wise 291 orthonormal matrix and $\tilde{\mathbf{R}}$ is a $(2 \times 2)$ upper triangular matrix 292 with positive real-valued diagonal entries formulated as

$$
\tilde{\mathbf{R}}=\left[\begin{array}{cc}
\tilde{R}_{1,1} & \tilde{R}_{1,2} \\
0 & \tilde{R}_{2,2}
\end{array}\right]
$$

Let $[\tilde{\mathbf{R}}]_{k}=\tilde{R}_{k, k}$ denote the $k t h$ diagonal entry of $\tilde{\mathbf{R}}$. Based 294 on this decomposition, another lower bound of the distance 295 $d_{\min }^{\text {joint }}$ in Eq. (18) can be formulated as

$$
\begin{aligned}
d_{\min }^{\text {joint }} & \geq d_{\min }^{\mathrm{QRD}-\text { bound }} \\
& =\min _{i, j=1, \cdots, L, i \neq j}\left\{[\tilde{\mathbf{R}}]_{\min }^{2}\right\} \min _{s_{a} \neq s_{b} \in \mathbb{S}}\left\|\left[s_{a}, s_{b}\right]\right\|_{F}^{2}, \\
& =\min _{i, j=1, \cdots, L, i \neq j}\left\{[\tilde{\mathbf{R}}]_{\min }^{2}\right\} d_{\min }^{\text {all }}
\end{aligned}
$$

\footnotetext{
${ }^{1}$ In SM, the transmit vector $\mathbf{x}$ only has a single non-zero element, hence the number of non-zero elements of the error vectors $\mathbf{e}_{i j}$ of SM is no more than 2.
} 
where $[\tilde{\mathbf{R}}]_{\min }^{2}$ is the minimum squared nonzero diagonal entry of the upper matrix $\tilde{\mathbf{R}}$, given by

$$
[\tilde{\mathbf{R}}]_{\min }=\min \left\{\tilde{R}_{1,1}, \tilde{R}_{2,2}\right\} .
$$

Lemma 1: For an $\left(N_{r} \times 2\right)$-element full column-rank matrix $\mathbf{H}_{u, i j}$ associated with its minimum squared singular non-zero value $\lambda_{\min }^{2}\left(\mathbf{H}_{u, i j}\right)$ for SVD and its minimum squared diagonal non-zero entry $[\tilde{\mathbf{R}}]_{\min }^{2}$ of $\tilde{\mathbf{R}}$ for $\mathrm{QRD}$, respectively, the inequality $[\tilde{\mathbf{R}}]_{\min }^{2} \geq \lambda_{\min }^{2}\left(\mathbf{H}_{u, i j}\right)$ is satisfied.

According to the analysis process in Section III of [38], the formulation of Lemma 1 is straightforward. As a result, the lower bound of Eq. (22) achieved by the QRD is tighter than that of the SVD algorithm in Eq. (18).

To derive an even tighter upper QRD bound than that of Eq. (22), the permutation matrix $\Pi_{m}$ can be invoked for calculating $d_{\min }^{\text {joint }}$ of Eq. (22) as

$$
d_{\min }^{\text {joint }}=\min _{\substack{i, j=1, \cdots, L, i \neq j, s_{a}, s_{b} \in \mathbb{S}}}\left\|\left[\mathbf{h}_{u}(i),-\mathbf{h}_{u}(j)\right] \boldsymbol{\Pi}_{m} \boldsymbol{\Pi}_{m}^{-1}\left[s_{a}, s_{b}\right]^{T}\right\|_{F}^{2},
$$

where $\boldsymbol{\Pi}_{m}$ is an orthogonal matrix satisfying $\boldsymbol{\Pi}_{m}^{-1}=\boldsymbol{\Pi}_{m}^{T}$. Since the size of the channel matrix $\mathbf{H}_{u, i j}=\left[\mathbf{h}_{u}(i),-\mathbf{h}_{u}(j)\right]$ is $N_{r} \times 2$, we only have two legitimate permutation matrices $\Pi_{m} \in \mathbb{C}^{2 \times 2}, m=1,2$, namely

$$
\boldsymbol{\Pi}_{1}=\left[\begin{array}{ll}
1 & 0 \\
0 & 1
\end{array}\right] \text { and } \boldsymbol{\Pi}_{2}=\left[\begin{array}{ll}
0 & 1 \\
1 & 0
\end{array}\right] .
$$

For each matrix $\Pi_{m}$, similar to Eq. (22), the corresponding QRD-based bound is

$$
\begin{aligned}
d_{\min }^{\text {joint }} & \geq \min _{i, j=1, \cdots, L, i \neq j}\left\{\left[\tilde{\mathbf{R}}_{m}\right]_{\min }^{2}\right\} \min _{s_{a}, s_{b} \in \mathbb{S}}\left\|\boldsymbol{\Pi}_{m}^{T}\left[s_{a}, s_{b}\right]^{T}\right\|_{F}^{2} \\
& =\left[\tilde{\mathbf{R}}_{m}\right]_{\min }^{2} d_{\min }^{\text {all }},
\end{aligned}
$$

where $\tilde{\mathbf{R}}_{m}$ is the upper triangular part of the QRD of the equivalent matrix $\mathbf{H}_{u, i j} \boldsymbol{\Pi}_{m}$. Note in Eq. (26) that the permutation matrix does not change the distance of $\left\|\boldsymbol{\Pi}_{m}^{T}\left[s_{a}, s_{b}\right]\right\|_{F}^{2}$ and we have $\min _{s_{a}, s_{b} \in \mathbb{S}}\left\|\Pi_{m}^{T}\left[s_{a}, s_{b}\right]^{T}\right\|_{F}^{2}=$ $\min _{s_{a}, s_{b} \in \mathbb{S}}\left\|\left[s_{a}, s_{b}\right]^{T}\right\|_{F}^{2}=d_{\min }^{\text {all }}$. For the permutation matrices given in Eq. (25), we can obtain two different values $\left[\tilde{\mathbf{R}}_{m}\right]_{\text {min }}$ $(m=1,2)$, which are given by $\left[\tilde{\mathbf{R}}_{1}\right]_{\min }=\min \left\{\tilde{R}_{1,1}\left(\boldsymbol{\Pi}_{1}\right)\right.$, $\left.\tilde{R}_{2,2}\left(\boldsymbol{\Pi}_{1}\right)\right\}$ and $\left[\tilde{\mathbf{R}}_{2}\right]_{\min }=\min \left\{\tilde{R}_{1,1}\left(\boldsymbol{\Pi}_{2}\right), \tilde{R}_{2,2}\left(\boldsymbol{\Pi}_{2}\right)\right\}$. Here, $\tilde{R}_{1,1}\left(\boldsymbol{\Pi}_{m}\right)$ and $\tilde{R}_{2,2}\left(\boldsymbol{\Pi}_{m}\right), m=1,2$ are the diagonal elements of $\tilde{\mathbf{R}}_{m}$.

Remark: The bound of Eq. (22) constitutes a special case of the bound of Eq. (26), which can be obtained by setting $m=1$.

Based on Eq. (26), an improved QRD-based upper bound of the distance $d_{\min }^{\text {joint }}$ is given by

$$
\begin{aligned}
d_{\min }^{\text {joint }} & \geq d_{\min }^{\mathrm{QRD}} \\
& =\min _{i, j=1, \cdots, L, i \neq j}\left\{\left[\tilde{\mathbf{R}} Q R Q_{-} P\right]_{\min }^{2}\right\} d_{\min }^{\text {all }} .
\end{aligned}
$$

where we have $\left[\tilde{\mathbf{R}}_{Q R Q_{-} P}\right]_{\min }^{2}=\max \left\{\left[\tilde{\mathbf{R}}_{1}\right]_{\min }^{2},\left[\tilde{\mathbf{R}}_{2}\right]_{\min }^{2}\right\}$.

Lemma 2: For an $\left(N_{r} \times 2\right)$-element full column-rank 332 matrix $\mathbf{H}_{u, i j}$ having a minimum squared diagonal non-zero 333 entry $[\tilde{\mathbf{R}}]_{\min }^{2}$ for its $\mathrm{QRD}$ and a value of $\left[\tilde{\mathbf{R}} Q R Q_{-} P\right]_{\min }^{2}=334$ $\max \left\{\left[\tilde{\mathbf{R}}_{1}\right]_{\min }^{2},\left[\tilde{\mathbf{R}}_{2}\right]_{\min }^{2}\right\}$ based on the pair of legitimate permuta- 335 tion matrices $\boldsymbol{\Pi}_{m} \in \mathbb{C}^{2 \times 2}, m=1,2$, respectively, the inequal- 336 ity $\left[\tilde{\mathbf{R}}_{Q R Q_{-} P}\right]_{\min }^{2} \geq[\tilde{\mathbf{R}}]_{\min }^{2}$ is satisfied. $\quad 337$

Since we have $[\tilde{\mathbf{R}}]_{\min }^{2}=\left[\tilde{\mathbf{R}}_{1}\right]_{\min }^{2}$, Lemma 2 can be obtained. 338

2) The Proposed QRD-Based ED-TAS: According to 339 Lemma 2, the QRD bound of Eq. (27) is tighter than that 340 of Eq. (22). Hence, we use this tighter bound to derive the 341 proposed QRD-based ED-TAS as

$$
\mathbf{H}_{\hat{u}}=\arg \max _{u \in\left\{1, \cdots, N_{U}\right\}}\left\{d_{\min }^{\text {signal }}, d_{\min }^{\text {spatial }}, d_{\min }^{\mathrm{QRD}-\text { bound_P }}\right\} .
$$

Note that the complexity of the QRD-based TAS is domi- 343 nated by the computation of $\left[\tilde{\mathbf{R}}_{m}\right]_{\min }$. In general, the full QRD 344 can be adopted in Eq. (26) for solving Eq. (27). However, this 345 may impose a high complexity. In order to reduce this com- 346 plexity, for a fixed channel $\mathbf{H}_{u, i j}$, we found that the value of 347 $\left[\tilde{\mathbf{R}}_{m}\right]_{\text {min }}$ only depends on the diagonal entries of $\tilde{\mathbf{R}}_{m}$, namely 348 $\tilde{R}_{k, k}\left(\boldsymbol{\Pi}_{m}\right)(k=1,2)$, which can be directly calculated as [38] 349

$$
\left[\tilde{\mathbf{R}}_{m}\right]_{k}=\tilde{R}_{k, k}\left(\boldsymbol{\Pi}_{m}\right)=\sqrt{\frac{\operatorname{det}\left[(\mathbf{G}(1: k))^{H} \mathbf{G}(1: k)\right]}{\operatorname{det}\left[(\mathbf{G}(1: k-1))^{H} \mathbf{G}(1: k-1)\right]}},
$$

where $\mathbf{G}(1: k)$ denotes a matrix consisting of the first $k 350$ columns of $\mathbf{H}_{u, i j} \boldsymbol{\Pi}_{m}$. In the classic V-BLAST systems, the cal- 351 culation of Eq. (29) suffers from the problem of having a high 352 complexity [38]. In SM, the number of non-zero elements of 353 the error vectors of SM is up to 2. This sparse character leads 354 to the simple sub-matrix $\mathbf{H}_{u, i j}=\left[\mathbf{h}_{u}(i),-\mathbf{h}_{u}(j)\right] \in \mathbb{C}^{N_{r} \times 2}$ and 355 hence the values of $\tilde{R}_{k, k}\left(\boldsymbol{\Pi}_{m}\right)(m=1,2, k=1,2)$ are given by 356

$$
\begin{aligned}
& \tilde{R}_{1,1}\left(\boldsymbol{\Pi}_{1}\right)=\sqrt{\left\|\mathbf{h}_{u}(i)\right\|_{F}^{2}}, \\
& \tilde{R}_{2,2}\left(\boldsymbol{\Pi}_{1}\right)=\sqrt{\frac{\left\|\mathbf{h}_{u}(i)\right\|_{F}^{2}+\left\|\mathbf{h}_{u}(j)\right\|_{F}^{2}-2 \mathcal{R}\left\{\mathbf{h}_{u}(i)^{H} \mathbf{h}_{u}(j)\right\}}{\left\|\mathbf{h}_{u}(i)\right\|_{F}^{2}}} \\
& \tilde{R}_{1,1}\left(\boldsymbol{\Pi}_{2}\right)=\sqrt{\left\|\mathbf{h}_{u}(j)\right\|_{F}^{2}}
\end{aligned}
$$

and

$$
\tilde{R}_{2,2}\left(\boldsymbol{\Pi}_{2}\right)=\sqrt{\frac{\left\|\mathbf{h}_{u}(i)\right\|_{F}^{2}+\left\|\mathbf{h}_{u}(j)\right\|_{F}^{2}-2 \mathcal{R}\left\{\mathbf{h}_{u}(i)^{H} \mathbf{h}_{u}(j)\right\}}{\left\|\mathbf{h}_{u}(j)\right\|_{F}^{2}}}
$$

The complexity of our proposed QRD-TAS of Eq. (28) 358 is dominated by the computation of $\tilde{R}_{k, k}\left(\boldsymbol{\Pi}_{m}\right), m=1,2$. In 359 $\mathrm{SM}$, these values only depend on the values of $\left\|\mathbf{h}_{u}(i)\right\|_{F}^{2}, \quad 360$ $\left\|\mathbf{h}_{u}(j)\right\|_{F}^{2}$ and $\mathbf{h}_{u}(i){ }^{H} \mathbf{h}_{u}(j)$, which are elements of the matrix 361 $\mathbf{H}^{H} \mathbf{H}$, as shown in Eqs. (30)-(33). Based on this observa- 362 tion, we can calculate the values of $\tilde{R}_{k, k}\left(\boldsymbol{\Pi}_{m}\right), m=1,2$ by 363 reusing these elements for the different TAS candidates $\mathbf{H}_{u}, 364$ 
TABLE I

COMPleXity COMPARISON OF DIFFERENT TAS ALgORITHMS FOR SM SySTEMS

\begin{tabular}{|c|c|c|}
\hline TAS algorithm & ED Optimality & Computational complexity \\
\hline Exhaustive ED-TAS [13] & optimal & $\frac{N_{t}\left(N_{t}-1\right)}{2}\left(5 N_{r}-1\right) M^{2}$ \\
\hline $\begin{array}{l}\text { Maximum-norm based } \\
\text { TAS of [21] }\end{array}$ & sub-optimal & $2 N_{t} N_{r}-N_{t}$ \\
\hline $\begin{array}{l}\text { Minimum-correlation } \\
\text { based TAS of [15] }\end{array}$ & sub-optimal & $2 N_{t}^{2} N_{r}-N_{t}^{2}+\frac{3}{2} N_{t}\left(N_{t}-1\right)$ \\
\hline $\begin{array}{l}\text { Conventional QRD-based } \\
\text { ED-TAS of [24] }\end{array}$ & optimal & $\begin{array}{c}2 N_{t} N_{r}-N_{t}+32 N_{t}\left(N_{t}-1\right)\left(N_{r}-\frac{2}{3}\right) \frac{M}{N_{A P M}} \\
\left(N_{A P M}=M \text { for PSK, } N_{A P M}=4 \text { for QAM }\right)\end{array}$ \\
\hline $\begin{array}{l}\text { Conventional SVD-based } \\
\text { ED-TAS of [23] }\end{array}$ & sub-optimal & $2 N_{t} N_{r}-N_{t}+\frac{19}{2} N_{t}\left(N_{t}-1\right)\left(N_{r}-\frac{1}{3}\right)$ \\
\hline Simplified SVD-TAS [25] & sub-optimal & $\frac{N_{t}\left(N_{t}-1\right)}{2}\left(2 N_{r}+11\right)+N_{t}\left(2 N_{r}-1\right)$ \\
\hline $\begin{array}{l}\text { Proposed QRD-based } \\
\text { ED-TAS }\end{array}$ & sub-optimal & $2 N_{t}^{2} N_{r}+\frac{3}{2} N_{t}\left(N_{t}-1\right)$ \\
\hline \multirow{2}{*}{$\begin{array}{l}\text { Proposed EVM-based } \\
\text { ED-TAS }\end{array}$} & $M$-PSK: optimal & $2 N_{t}^{2} N_{r}-N_{t}^{2}+\frac{1}{2} N_{t}\left(N_{t}-1\right)(M+7)$ \\
\hline & $M$-QAM $\left\{\begin{array}{c}\text { sub-optimal, } K<v \\
\text { optimal, } K=v\end{array}\right.$ & $2 N_{t}^{2} N_{r}-N_{t}^{2}+\frac{15}{2} G N_{t}\left(N_{t}-1\right)$ \\
\hline Exhaustive TAS\&PA & - & $\left(\begin{array}{c}N_{t} \\
L\end{array}\right) C_{\mathrm{PA}}$ \\
\hline Low-complexity TAS\&PA & 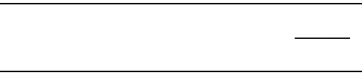 & $C_{\mathrm{TAS}}+C_{\mathrm{PA}}=\left\{\begin{array}{c}C_{\mathrm{PQRD}}+C_{\mathrm{PA}} \\
C_{\mathrm{EVM}}+C_{\mathrm{PA}}\end{array}\right.$ \\
\hline
\end{tabular}

hence the resultant complexity is considerably reduced compared to the conventional QRD-based ED-TAS, as will show in Table I.

To confirm the benefits of the QRD-based bound derived in Eq. (27), Fig. 2 shows the BER performance of the proposed QRD-based ED-TAS algorithm in contrast to the existing SVDbased ED-TAS of [23]. Moreover, we add the performance of the norm-based TAS of [13] and of the exhaustive-search based optimal ED-TAS of [21] as benchmarks. In Fig. 2, the number of TAs is set to $N_{t}=4$, where $L=2$ out of $N_{t}=$ 4 TAs were selected in these TAS algorithms. As expected, since the proposed QRD-based ED-TAS has a tighter bound, in Fig. 2 it performs better than the SVD-based ED-TAS. Quantitatively, observe in Fig. 2 that this scheme provides an SNR gain of about $1.2 \mathrm{~dB}$ over the SVD-based ED-TAS at the BER of $10^{-5}$. In Fig. 2, we also observe that the QRDbased ED-TAS achieves a near-optimum performance, where the performance gap between the proposed QRD-based EDTAS and the exhaustive-search-based optimal ED-TAS is only about $0.2 \mathrm{~dB}$. We will provide more detailed comparisons about the BER and the complexity issues in Section VI.

\section{B. The Proposed EVM-Based ED-TAS}

In this section, for striking a further flexible tradeoff in terms of the BER attained and the complexity imposed, we propose an EVM-based ED-TAS algorithm. The proposed EVM-TAS directly calculates the value of $d_{\min }\left(\mathbf{H}_{u}\right)$ for the specific TAS candidate $\mathbf{H}_{u}$, rather than exploiting the equivalent decision metric of Eq. (13) or the estimated bound of (18). Specifically, we will derive simple optimization metrics for both PSK and QAM constellations, where the error-vector selection probability is exploited for reducing the search space.

1) The Calculation of $d_{\min }\left(\mathbf{H}_{u}\right)$ in EVM-Based ED-TAS: Specifically, the $M$-PSK constellation can be expressed as $\mathbb{S}_{P S K}=\left\{e^{j \frac{2 m \pi}{M}}, m=0, \cdots, M-1\right\}$, and the symbols of the rectangular $M=4^{k} \mathrm{QAM}$ constellation belong to the set of [36]

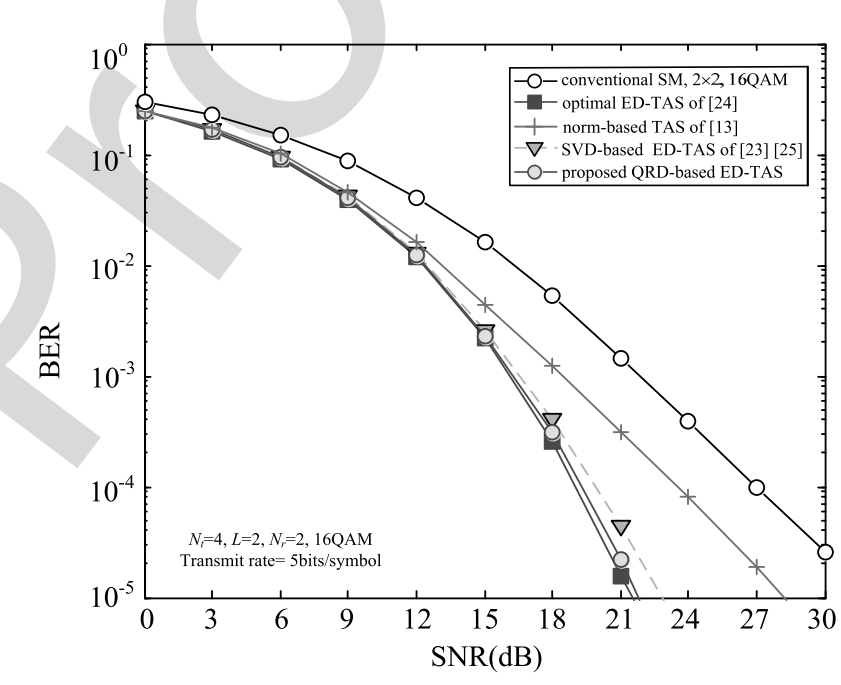

Fig. 2. BER performance comparison of the existing TAS algorithms and the proposed QRD-based ED-TAS algorithm. The setup of the simulation is based on $N_{t}=4, N_{r}=2, L=2$ and 16-QAM. The transmit rate is 5 bits/symbol.

$$
\mathbb{S}_{Q A M}=\frac{1}{\sqrt{\beta_{k}}}\{a+b j, a-b j,-a+b j,-a-b j\},
$$

where we have $\beta_{k}=\frac{2}{3}\left(4^{k}-1\right)$ and $a, b \in\left\{1,3, \cdots, 2^{k}-1\right\} .401$ Similar to Eq. (14), the calculation of $d_{\min }\left(\mathbf{H}_{u}\right)$ is parti- 402 tioned into three cases: $d_{\min }^{\text {signal }}, d_{\min }^{\text {spatial }}$ and $d_{\min }^{\text {joint }}$. As shown in 403 Eqs. (15)-(16), $d_{\mathrm{min}}^{\text {signal }}$ depends the minimum distance of the 404 $\mathrm{APM} d_{\min }^{\mathrm{APM}}$ as [39]

$$
d_{\mathrm{min}}^{\mathrm{APM}}=\left\{\begin{array}{lll}
4 \sin ^{2}(\pi / M) & \text { for } & M-\mathrm{PSK} \\
\frac{4}{\beta_{k}} & \text { for } & M-\mathrm{QAM}
\end{array},\right.
$$

while $d_{\text {min }}^{\text {spatial }}$ relies on the minimum squared modulus value 406 $d_{\mathrm{min}}^{\text {Modulus }}$ of the APM constellation as

$$
d_{\min }^{\text {Modulus }}=\left\{\begin{array}{lll}
1 & \text { for } & M-\mathrm{PSK} \\
\frac{2}{\beta_{k}} & \text { for } & M-\mathrm{QAM}
\end{array} .\right.
$$


Based on Eqs. (35) and (36), the complexity of computing the values of $d_{\mathrm{min}}^{\text {signal }}$ and $d_{\mathrm{min}}^{\text {spatial }}$ in Eqs. (15)-(16) may be deemed negligible. Hence, we only have to reduce the complexity of computing $d_{\mathrm{min}}^{\text {joint }}$, which can be achieved as follows:

$$
\begin{aligned}
& d_{\min }^{\text {joint }-E V M}=\min _{\substack{i, j=1, \cdots, L, i \neq j \\
s_{a}, s_{b} \in \mathbb{S}}}\left\|\mathbf{h}_{u}(i) s_{a}-\mathbf{h}_{u}(j) s_{b}\right\|_{F}^{2} \\
& =\min _{\substack{i, j=1, \cdots, L, i \neq j, s_{a}, s_{b} \in \mathbb{S}}}\left|s_{a}\right|^{2}\left\|\mathbf{h}_{u}(i)\right\|_{F}^{2}+\left|s_{b}\right|^{2}\left\|\mathbf{h}_{u}(j)\right\|_{F}^{2}-2 m_{\mathrm{APM}},
\end{aligned}
$$

where we have $m_{\mathrm{APM}}=\mathcal{R}\left\{s_{a}^{H} s_{b} \mathbf{h}_{u}(i)^{H} \mathbf{h}_{u}(j)\right\}$, which relies on the specific APM scheme adopted. Next, we will derive the simplified metrics $d_{\min }^{\text {joint-EVM }}$ for the general family of $M$-PSK and $M$-QAM modulated SM systems.

2) Simplification for $M$-PSK Schemes: For a pair of $M$-PSK symbols $s_{a}=e^{j \frac{2 a \pi}{M}}$ and $s_{b}=e^{j \frac{2 b \pi}{M}}$, the possible values of $s_{a}^{H} s_{b}$ obey $e^{j \frac{2(b-a) \pi}{M}},(b-a) \in\{-(M-1), \cdots,(M-1)\}$. As a result, $m_{\mathrm{APM}}$ of the general $M$-PSK scheme obeys:

$$
m_{\mathrm{APM}} \in\left\{\mathcal{R}\left\{\mathbf{h}_{u}(i){ }^{H} \mathbf{h}_{u}(j)\right\} \cos \theta_{n}-\mathcal{J}\left\{\mathbf{h}_{u}(i){ }^{H} \mathbf{h}_{u}(j)\right\} \sin \theta_{n}\right\},
$$

where $\theta_{n}=\frac{2 n \pi}{M}, n=-(M-1), \cdots,(M-1)$. Since the minimum ED is considered in Eq (37), only the maximum value of $m_{\mathrm{APM}}$ needs to be considered, which is given by Eq. (39), shown at the bottom of the page. As shown in Eq. (39), the number of possible $\theta_{n}$ values is reduced from $2 M-1$ to $\frac{M}{4}+1$. According to Eq. (39), $\left|s_{a}\right|^{2}=1$ and $\left|s_{b}\right|^{2}=1$, the distance $d_{\min }^{\text {joint }-E V M}$ of Eq. (37) is simplified for $M$-PSK as follows:

$$
d_{\min }^{\text {joint }-E V} \stackrel{\substack{i, j=1, \cdots, L \\ i \neq j}}{=} \min _{\substack{i, \cdots \\ i n}}\left\|\mathbf{h}_{u}(i)\right\|_{F}^{2}+\left\|\mathbf{h}_{u}(j)\right\|_{F}^{2}-2 m_{M-\operatorname{PSK}}\left(\mathbf{H}_{u}\right) .
$$

Example: The constellation points $s_{a}$ and $s_{b}$ of BPSK and QPSK modulation schemes belong to the set $\mathbb{S}_{\mathrm{BPSK}}=\{ \pm 1\} \quad$ and $\quad \mathbb{S}_{\mathrm{QPSK}}=\{ \pm 1, \pm j\}$, respectively. Based on Eq. (39), the corresponding optimized metrics $m_{M-\mathrm{PSK}}\left(\mathbf{H}_{u}\right)=\max m_{\mathrm{APM}}$ are simplified to $m_{2-\mathrm{PSK}}\left(\mathbf{H}_{u}\right)=\left|\mathcal{R}\left\{\mathbf{h}_{u}(i)^{H} \mathbf{h}_{u}(j)\right\}\right|$ and $m_{4-\mathrm{PSK}}\left(\mathbf{H}_{u}\right)=$ $\max \left\{\left|\mathcal{R}\left\{\mathbf{h}_{u}(i)^{H} \mathbf{h}_{u}(j)\right\}\right|,\left|\mathcal{J}\left\{\mathbf{h}_{u}(i){ }^{H} \mathbf{h}_{u}(j)\right\}\right|\right\}$, respectively.

As shown in Eqs. (37)-(40), since we have $\left|s_{a}\right|^{2}=1,\left|s_{b}\right|^{2}=$ 1 and a reduced set $s_{a}^{H} s_{b}$ for $M$-PSK constellation, the complexity of calculating $d_{\mathrm{min}}^{\text {joint }-E V M}$ is low, as it will be shown in Table I.

3) Simplification for M-QAM Schemes: When $M$-QAM constellations are considered, the calculation of $d_{\min }^{\text {joint }-E V M}$ in Eq. (37) becomes substantially complicated, because there are many combinations of the values of $\left|s_{a}\right|^{2},\left|s_{b}\right|^{2}$ and $s_{a}^{H} s_{b}$ in Eq. (37), which lead to different received SM-symbol distances. To derive a simplified optimized metrics for $M$-QAM, we first introduce the following Lemma.

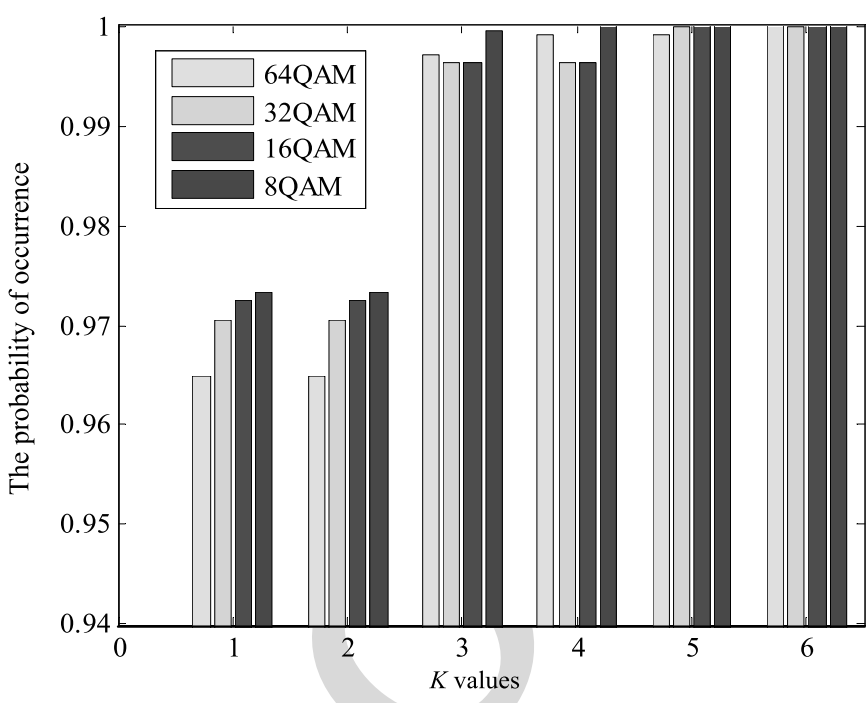

Fig. 3. The statistical probability of the norm error vectors relying on $K$ minimum moduli, yielding the optimal ED-TAS solution, where the system setup is $N_{t}=4, N_{r}=2$ and $L=2$.

Lemma 3: It is highly likely that an error vector associated 445 with a small norm value yields the FD value of Eq. (9). Thus, 446 the search space to be evaluated for finding the FD can be 447 reduced to a few dominant error vectors having small norm 448 values.

Proof: Based on the Rayleigh-Ritz theorem of [37], for 450 a fixed channel matrix $\mathbf{H}_{u, i j}$ and a given error vector $\mathbf{e}_{i j}, 451$ the distance amongst the received symbols is bounded by 452 $\lambda_{\max }^{2}\left(\mathbf{H}_{u, i j}\right)\left\|\mathbf{e}_{i j}\right\|^{2} \geq\left\|\mathbf{H}_{u} \mathbf{e}_{i j}\right\|^{2} \geq \lambda_{\min }^{2}\left(\mathbf{H}_{u, i j}\right)\left\|\mathbf{e}_{i j}\right\|^{2}$, where 453 $\lambda_{\max }^{2}\left(\mathbf{H}_{u, i j}\right)$ is the maximum squared singular value of the sub- 454 matrix $\mathbf{H}_{u, i j}=\left[\mathbf{h}_{u}(i),-\mathbf{h}_{u}(j)\right]$. It may be readily shown that 455 the values of $\lambda_{\max }^{2}\left(\mathbf{H}_{u, i j}\right)$ and $\lambda_{\min }^{2}\left(\mathbf{H}_{u, i j}\right)$ are constants for 456 a fixed channel realization $\mathbf{H}_{u, i j}$, while the value of $\left\|\mathbf{e}_{i j}\right\|^{2} 457$ depends on the specific APM constellation points. Based on the 458 bound above, it is highly likely that an $\mathbf{e}_{i j}$ with a small norm 459 yields low upper bound and lower bound. Hence it has a high 460 probability of generating the FD value, as it will be exemplified 461 in Fig. 3.

462

Based on Lemma 3, for the sake of striking a beneficial 463 trade-off between the BER performance and complexity for 464 $M$-QAM, the search space is limited to the error vectors hav- 465 ing small modulus values and only these vectors are utilized to 466 compute the FD metric. Specifically, we first evaluate all possi- 467 ble modulus values $T_{1}, T_{2}, T_{3}, \cdots, T_{v}$ of all the legitimate error 468 vectors $\mathbf{e}_{i j}$, then we find the $K$ smallest $T_{K}$ from the full set 469 of $\left\{T_{1}, T_{2}, T_{3}, \cdots, T_{v}\right\}$ and only consider the set of $\mathbf{e}_{i j}$ having 470 moduli lower than $T_{K}$ to compute $d_{\min }\left(\mathbf{H}_{u}\right)$. In this process, 471 the error vectors can be divided into the pair of sub-sets $\mathbb{D}_{1}$ and 472 $\mathbb{D}_{2}$ based on their sparsity, where $\mathbb{D}_{1}$ contains the error vectors, 473 which have only a single non-zero element, while $\mathbb{D}_{2}$ contains 474

$$
\begin{aligned}
m_{M-\mathrm{PSK}}\left(\mathbf{H}_{u}\right)=\max _{n} m_{\mathrm{APM}} & =\max _{n \in\{-(M-1), \cdots, M-1\}}\left\{\mathcal{R}\left\{\mathbf{h}_{u}(i)^{H} \mathbf{h}_{u}(j)\right\} \cos \theta_{n}-\mathcal{J}\left\{\mathbf{h}_{u}(i)^{H} \mathbf{h}_{u}(j)\right\} \sin \theta_{n}\right\} \\
& =\max _{n \in\{0, \cdots, M / 4\}}\left\{\left|\mathcal{R}\left\{\mathbf{h}_{u}(i)^{H} \mathbf{h}_{u}(j)\right\} \cos \theta_{n}-\mathcal{J}\left\{\mathbf{h}_{u}(i)^{H} \mathbf{h}_{u}(j)\right\} \sin \theta_{n}\right|\right\}
\end{aligned} .
$$


the error vectors, which have two non-zero elements. As will be shown in our simulation results, $K=3$ is a good choice for diverse configurations, hence we only provide the simplified expressions of $d_{\min }^{\text {joint }-E V M}$ for $K \leq 3$ as follows.

For $K=1$, according to the $M$-QAM constellation of Eq. (34), only error vectors having $T_{1}=\sqrt{\frac{4}{\beta_{k}}}$ are considered and the associated sets $\mathbb{D}_{1}$ and $\mathbb{D}_{2}$ are given by $\mathbb{D}_{1}=\frac{1}{\sqrt{\beta_{k}}}\left\{ \pm 2 \mathbf{e}_{i}, \pm 2 \mathrm{j} \mathbf{e}_{i}\right\}, i=1, \cdots, L \quad$ and $\mathbb{D}_{2}=\frac{1}{\sqrt{\beta_{k}}}\left\{( \pm 1 \pm 1 \mathrm{j}) \mathbf{e}_{i}-( \pm 1 \pm 1 \mathrm{j}) \mathbf{e}_{j}\right\}, i, j=1, \cdots, L, i \neq$ $j$, respectively, where $\mathbf{e}_{i}$ and $\mathbf{e}_{j}$ are the active TA selection vectors in Eq. (2). Since only the minimum ED is considered, the set $\mathbb{D}_{1}$ can be reduced to $\mathbb{D}_{1}=\frac{1}{\sqrt{\beta_{k}}}\left\{2 \mathbf{e}_{i}\right\}, i=1, \cdots, L$. Moreover, based on the set $\mathbb{D}_{2}$, it is find that the elements $s_{a}$ and $s_{b}$ belong to the reduced set $\frac{1}{\sqrt{\beta_{k}}}\{ \pm 1 \pm 1 \mathrm{j}\}$ and we have $\left|s_{a}\right|^{2}=\frac{2}{\beta_{k}},\left|s_{b}\right|^{2}=\frac{2}{\beta_{k}}$ and $s_{a}^{H} s_{b} \in \frac{2}{\beta_{k}}\{ \pm 1, \pm 1 \mathrm{j}\}$. Substituting these values into Eq. (37), we get the simplified optimized metric for $K=1$ as

$$
d_{\min , K=1}^{\text {joint } E V M}=\min \frac{2}{\substack{i, j=1, \cdots, L, i \neq j,}}\left\|\mathbf{h}_{u}(i)\right\|_{F}^{2}+\frac{2}{\beta_{k}}\left\|\mathbf{h}_{u}(j)\right\|_{F}^{2}-2 m_{M-Q A M}^{K=1},
$$

where we have

$$
\begin{aligned}
& m_{M-Q A M}^{K=1}=\max m_{\mathrm{APM}} \\
& =\max \left\{\frac{2}{\beta_{k}}\left|\mathcal{R}\left\{\mathbf{h}_{u}(i)^{H} \mathbf{h}_{u}(j)\right\}\right|, \frac{2}{\beta_{k}}\left|\mathcal{J}\left\{\mathbf{h}_{u}(i)^{H} \mathbf{h}_{u}(j)\right\}\right|\right\} .
\end{aligned}
$$

For the case of $K=2$, all the error vectors $\mathbf{e}_{i j}$ having moduli lower than $T_{2}$ are used for FD calculation. Compared to $K=1$, we have to consider the added error vectors $\frac{1}{\sqrt{\beta_{k}}}\{ \pm 2 \pm$ $\left.2 \mathrm{j} \mathbf{e}_{i}\right\}(i=1, \cdots, L)$ having $T_{2}=\sqrt{\frac{8}{\beta_{k}}}$, which belong to $\mathbb{D}_{1}$ and do not change the set $\mathbb{D}_{2}$. After eliminating all collinear elements, the set $\mathbb{D}_{1}$ of $K=2$ is reduced to $\frac{1}{\sqrt{\beta_{k}}}\left\{2 \mathbf{e}_{i}, \pm 2 \pm\right.$ $\left.2 \mathbf{j}_{i}\right\}, i=1, \cdots, L$. Moreover, since only the minimum distance is investigated, the set is further reduced to $\mathbb{D}_{1}=$ $\frac{1}{\sqrt{\beta_{k}}}\left\{2 \mathbf{e}_{i}\right\}, i=1, \cdots, L$, which is the same as that of $K=1$. Therefore, the setups of $K=1$ and $K=2$ will provide the same FD $d_{\min }\left(\mathbf{H}_{u}\right)$.

Moreover, for the case of $K=3$, besides the error vectors $\mathbf{e}_{i j}$ for $K=2$, the error vectors having $T_{3}=\sqrt{\frac{10}{\beta_{k}}}$ should be considered, which are given by $\frac{1}{\sqrt{\beta_{k}}}\left\{( \pm 3 \pm 1 \mathrm{j}) \mathbf{e}_{i}-( \pm 1 \pm 1 \mathrm{j}) \mathbf{e}_{j},( \pm 1 \pm 3 \mathrm{j}) \mathbf{e}_{i}-( \pm 1 \pm 1 \mathrm{j}) \mathbf{e}_{j}\right\}$, $i, j=1, \cdots, L, i \neq j$. For these added error vectors, we have $s_{a}^{H} s_{b} \in \frac{1}{\beta_{k}}\{ \pm 2 \pm 4 \mathrm{j}, \pm 4 \pm 2 \mathrm{j}\}$ and two legitimate combinations of the values of $\left|s_{a}\right|^{2}$ and $\left|s_{b}\right|^{2}$ as: (1) $\left|s_{a}\right|^{2}=\frac{2}{\beta_{k}},\left|s_{b}\right|^{2}=\frac{10}{\beta_{k}}$ and (2) $\left|s_{a}\right|^{2}=\frac{10}{\beta_{k}},\left|s_{b}\right|^{2}=\frac{2}{\beta_{k}}$. For each combination, similar 511 to the process of Eqs. (41)-(42), we can substitute the values 512 of $\left|s_{a}\right|^{2},\left|s_{b}\right|^{2}$ and $s_{a}^{H} s_{b}$ into Eq. (37) and get the simplified 513 optimized metric for $K=3$ as

$$
d_{\min , K=3}^{\text {joint }-E V M}=\min \left\{d_{\min , K=1}^{\text {joint }-E V M}, d_{\min ,(1)}^{\text {joint }-E V M}, d_{\min ,(2)}^{\text {joint }-E V M}\right\}
$$

where $d_{\min ,(1)}^{\mathrm{join}-E V M}$ and $d_{\min ,(2)}^{\mathrm{joint}-E V M}$ are the simplified ED for the 515 above-mentioned two combinations, given by Eq. (44), shown 516 at the bottom of the page.

4) The Proposed EVM-Based ED-TAS: Based on the sim- 518 plified versions of $d_{\mathrm{min}}^{\text {joint }-E V M}$ for $M$-PSK and $M$-QAM 519 schemes derived in Eqs. (41) and (43), the solution of our 520 EVM-based ED-TAS algorithm is given by

$$
\mathbf{H}_{\hat{u}}=\arg \max _{u \in\left\{1, \cdots, N_{U}\right\}}\left\{d_{\text {min }}^{\text {signal }}, d_{\text {min }}^{\text {spatial }}, d_{\text {min }}^{\text {joint }-E V M}\right\} .
$$

Note that similar to the proposed QRD-TAS, the terms 522 $\left\|\mathbf{h}_{u}(i)\right\|_{F}^{2},\left\|\mathbf{h}_{u}(j)\right\|_{F}^{2}$ and $\mathbf{h}_{u}(i){ }^{H} \mathbf{h}_{u}(j)$ in Eqs. (40)-(44) are 523 elements of the matrix $\mathbf{H}^{H} \mathbf{H}$. Then, we can find the solu- 524 tion of Eq. (45) by reusing these elements for different TAS 525 candidates $\mathbf{H}_{u}$.

Fig. 3 shows the probability that the error vectors having the 527 minimum norm do result in finding the optimal ED-TAS solu- 528 tion as a function of $K$. For example, we have a probability 529 of $97 \%$ for 16-QAM modulated SM for $K=1$ using $N_{t}=4,530$ $L=2$ and $N_{r}=2$. Moreover, it is observed from Fig. 3 that 531 this probability is also high for other QAM schemes; hence the 532 EVM-based ED-TAS can be readily used in diverse scenarios. 533 In general, for striking a flexible BER vs complexity tradeoff, 534 we can adjust the parameter $K$ to reduce the search space to a 535 subset of the error vectors that may yield the optimal ED-TAS 536 solution with a high probability.

Note that in [17] a PEP-based TAS (PEP-TAS) algorithm was 538 proposed, which was based on a different search set reduction. 539 The main differences of the proposed EVM-TAS and the PEP- 540 TAS of [17] are:

- The PEP-TAS is based on the assumption that a smaller 542 APM symbol amplitude leads to a smaller distance $d_{\text {min }}^{\text {joint }}, 543$ whereas based on our analysis it is highly likely that an 544 error vector with a small norm yields the distance $d_{\min }^{\text {joint }} . \quad 545$

- Moreover, in EVM-TAS, we propose to use the parameter 546 $K$ for striking a flexible tradeoff between the conflicting 547 factors of the computational complexity imposed and the 548 attainable BER.

Remark: Compared to the EVM-TAS, the PEP-TAS con- 550 siders only the error vectors generated by $M$-QAM symbols 551 having the minimum amplitude. It can be shown that the non- 552 linear error vectors of the PEP-TAS are the same as those of the 553

$$
\left\{\begin{array}{l}
d_{\min ,(1)}^{\text {joint-EVM }}=\min _{\substack{i, j=1, \cdots, L \\
i \neq j}} \frac{2}{\beta_{k}}\left\|\mathbf{h}_{u}(i)\right\|_{F}^{2}+\frac{10}{\beta_{k}}\left\|\mathbf{h}_{u}(j)\right\|_{F}^{2}-2 m_{M-Q A M}^{K=3} \\
d_{\min ,(2)}^{\text {joint } E V M}=\min _{\substack{i, j=1, \cdots, L \\
i \neq j}} \frac{10}{\beta_{k}}\left\|\mathbf{h}_{u}(i)\right\|_{F}^{2}+\frac{2}{\beta_{k}}\left\|\mathbf{h}_{u}(j)\right\|_{F}^{2}-2 m_{M-Q A M}^{K=3} \\
m_{M-Q A M}^{K=3}=\max \frac{1}{\beta_{k}}\left\{\left|2 \mathcal{R}\left\{\mathbf{h}_{u}(i)^{H} \mathbf{h}_{u}(j)\right\}\right|+\left|4 J\left\{\mathbf{h}_{u}(i)^{H} \mathbf{h}_{u}(j)\right\}\right|,\left|4 \mathcal{R}\left\{\mathbf{h}_{u}(i)^{H} \mathbf{h}_{u}(j)\right\}\right|+\left|2 \mathcal{J}\left\{\mathbf{h}_{u}(i)^{H} \mathbf{h}_{u}(j)\right\}\right|\right\}
\end{array}\right.
$$




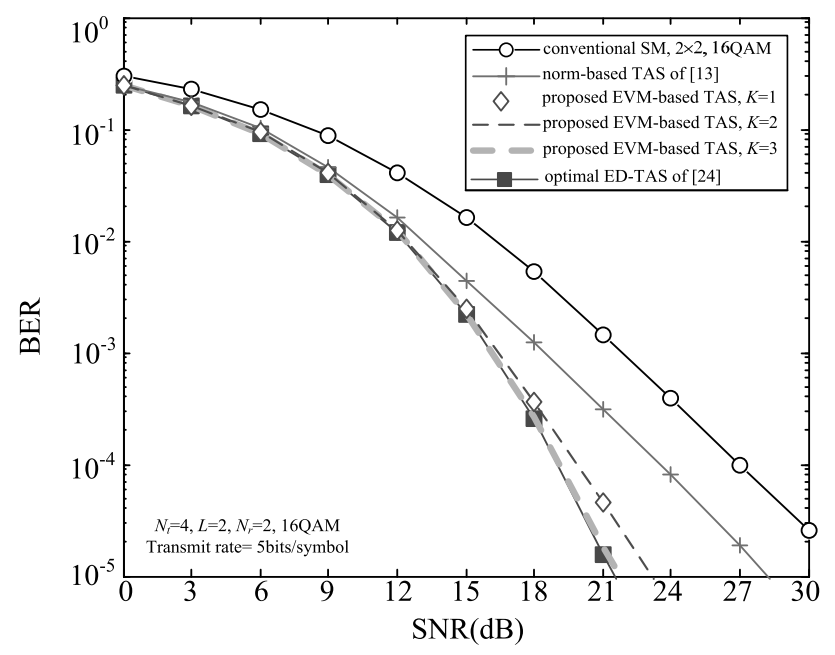

Fig. 4. BER performance comparison of the existing TAS algorithms and the proposed EVM-based TAS algorithm for $N_{t}=4, N_{r}=2,16 \mathrm{QAM}$ and $L=2$. The transmit rate is 5 bits/symbol.

EVM-TAS associated with $K=1$. Therefore, it can be viewed as a special case of EVM-TAS by setting $K=1$.

Fig. 4 shows our BER comparison for the existing TAS algorithms and the proposed EVM-TAS algorithm. The simulation parameters are the same as those of Fig. 2. Firstly, as proved in Section IV-B and observed in Fig. 3, the probability that the error vectors do indeed result in the optimal ED-TAS solution is the same for the cases of $K=1$ and $K=2$. Hence, they provide the same BER performance, as shown in Fig. 4. Furthermore, we observe in Fig. 3 that this probability is increased from 0.975 to 0.998 upon increasing $K$ from 1 to 3. As a result, in Fig. 4 the performance of the EVM-based ED-TAS associated with $K=3$ is improved compared to that scheme with $K=1$. Moreover, compared the results in Figs. 2 and 4, the EVM-based ED-TAS outperforms the SVD-based ED-TAS for $K=3$.

\section{JOINT TAS AND PA ALGORITHMS FOR SM}

Similar to the TAS technique, PA is another attractive link adaptation technique conceived for SM, which has been advocated in [7], [11], [28], [29]. The process of PA can be modeled by the PA matrix $\mathbf{P}$, which is given by

$$
\mathbf{P}=\operatorname{diag}\left\{p_{1}, \cdots, p_{q}, \cdots, p_{L}\right\},
$$

where $p_{q}$ controls the channel gain of the $q t h$ TA. Here, we let $\sum_{q=1}^{L} p_{q}^{2}=1$ for normalizing the transmit power. Based on our TAS algorithms, we propose a pair of combined algorithms for jointly considering the PA and TAS as follows:

\section{1) TAS\&PA}

- Step 1: Each $\left(N_{r} \times N_{t}\right)$ channel matrix $\mathbf{H}$ has $N_{U}=$ $\left(\begin{array}{c}N_{t} \\ L\end{array}\right)$ possible subchannel matrices $\mathbf{H}_{u}$, each of which corresponds to a specifically selected $\left(N_{r} \times\right.$ $L)$ MIMO channel. For each $\mathbf{H}_{u}$, we calculate the corresponding PA matrix $\mathbf{P}_{u}$ and its FD with the aid of the algorithm of [29].

- Step 2: The particular combinations of $\mathbf{H}_{u} \mathbf{P}_{u}(u=$ $\left.1, \cdots, N_{U}\right)$ constitute the legitimate TAS\&PA candidates. Let us interpret the matrices $\mathbf{H}_{u} \mathbf{P}_{u} 588$ ( $\left.u=1, \cdots, N_{U}\right)$ as being the equivalent channel 589 matrices of Section IV and select the specific can- 590 didate with the maximum free distance as the final 591 solution.

Since for each channel realization $\mathbf{H}$, there are $N_{U}$ pos- 593 sible PA matrices $\mathbf{P}_{u}\left(u=1, \cdots, N_{U}\right)$, we have a high 594 computational complexity if $N_{U}$ is high. Next, we intro- 595 duce a lower-complexity solution for this joint TAS and 596 PA algorithm.

2) Low-complexity TAS\&PA the proposed low-complexity QRD-based ED-TAS 600 or the EVM-based ED-TAS algorithm to select a 601 particular subset of TAs from the set of options, 602 which corresponds to $\mathbf{H}_{\hat{u}}$.

- Step 2: Calculate the power weights for the selected 604 TAs, which can be represented by the PA matrix $\mathbf{P}_{\hat{u}}$. 605 During this step, the low-complexity PA algorithm 606 of [29] can be invoked. In the simple TAS\&PA, the 607 PA matrix only has to be calculated once, hence the 608 associated complexity is low.

\section{Simulation Results}

In this section, we provide simulation results for further char- 611 acterizing the proposed QRD-based ED-TAS, EVM-based ED- 612 TAS and TAS\&PA schemes for transmission over frequency- 613 flat fading MIMO channels. For comparison, these performance 614 results are compared to various existing TAS-SM schemes of 615 [13], [21], [23], [25], to the classic TAS/maximal-ratio combin- 616 ing (TAS/MRC) schemes of [40], as well as to the TAS\&PA 617 aided V-BLAST of [32]. In our simulations, the single-stream 618 ML detector of [34], [35] is utilized.

\section{A. BER Comparisons of Different TAS Algorithms for SM}

In Fig. 5, we compare the BER performance of various TAS- 621 SM schemes for 4 bits/symbol associated with $N_{t}=8, L=4,622$ $N_{r}=4$ and QPSK. We also considered the conventional single- 623 RF based TAS/MRC arrangement of [40] as benchmarker. As 624 seen from Fig. 5, the proposed QRD-based ED-TAS outper- 625 forms the conventional SVD-based ED-TAS of [23], as also 626 formally shown in Fig. 2. Moreover, as expected, in Fig. 5627 the EVM-based TAS is capable of achieving the same per- 628 formance as the optimal ED-TAS of [21]. We also confirm 629 that our proposed EVM-based ED-TAS schemes outperform 630 the norm-based TAS of [13] and the QRD-based ED-TAS pro- 631 posed for PSK modulation. These results are consistent with the 632 analysis results in Section IV, where the EVM-based TAS has 633 considered all legitimate error vectors for simplifying $d_{\min }^{\text {joint }}$ in 634 Eq. (40), while the QRD-based ED-TAS may achieve uncorrect 635 estimation of $d_{\mathrm{min}}^{\text {joint }}$ due to the employment of lower bound of 636 Eq. (27).

Fig. 5 also shows that our new TAS-SM schemes outper- 638 form the TAS/MRC scheme of [40]. The main reason behind 639 the poorer performance of TAS/MRC is the employment of 640 a higher modulation order required for achieving the same 641 


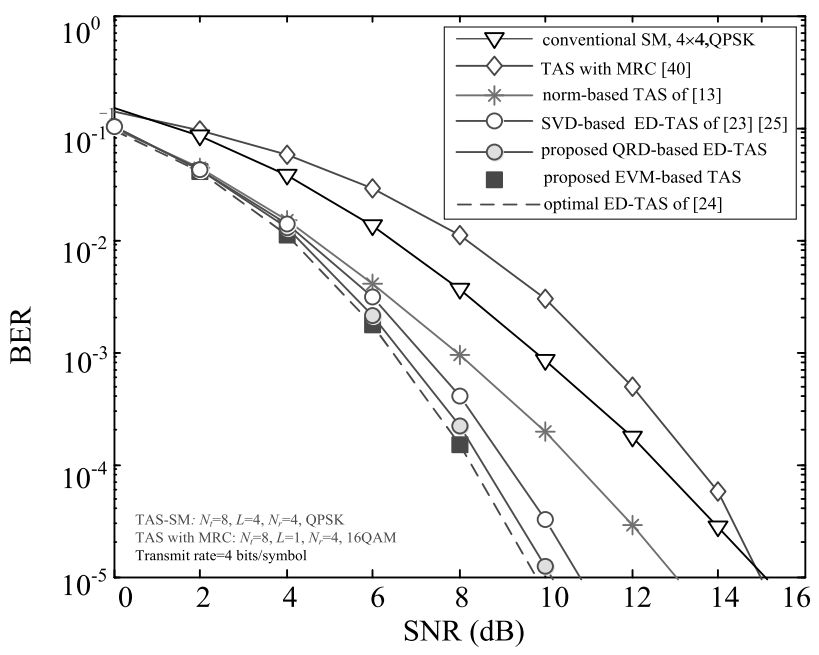

Fig. 5. BER comparison at $m=4$ bits/symbol for the proposed TAS-SM schemes, the existing TAS-SM schemes and the classic TAS/MRC scheme having $N_{t}=8$ and $L=4$.

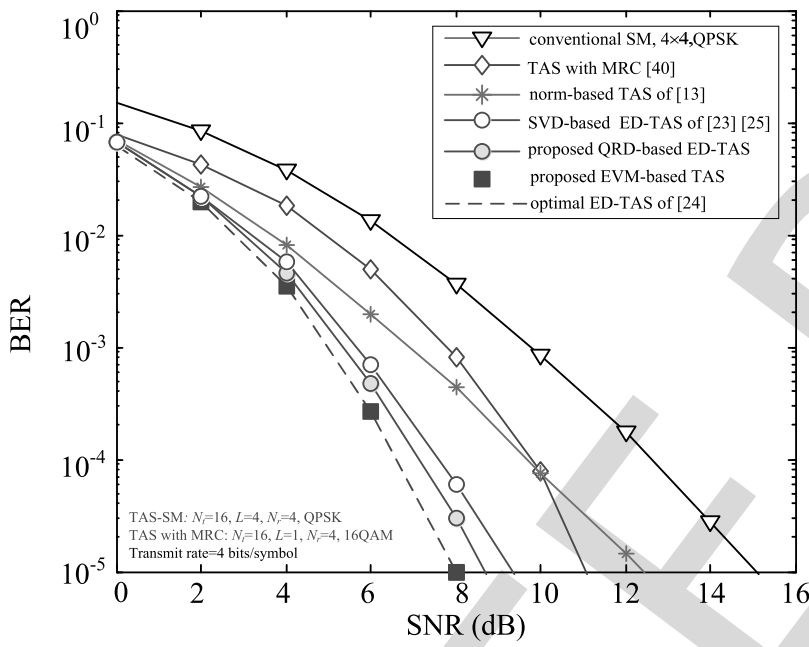

Fig. 6. BER comparison at $m=4$ bits/symbol for the proposed TAS-SM schemes, the existing TAS-SM schemes and the classic TAS/MRC scheme having $N_{t}=16$ and $L=4$.

throughput as our SM-based schemes. Note that this benefit depends on the particular MIMO setups. To be specific, as noted in [23], the TAS-SM and the TAS/MRC schemes exhibit different BER advantages for different system setups. However, similar to the results achieved in [23], our new TAS-SM schemes strike an attractive tradeoff between the complexity and the BER attained. The above-mentioned trends of these proposed TAS-SM schemes are also confirmed in Fig. 6, where the number $N_{t}$ of TAs increases from 8 to 16 .

In Fig. 7, a spatially correlated MIMO channel model characterized by $\mathbf{H}^{\text {corr }}=\mathbf{R}_{r}^{1 / 2} \mathbf{H} \mathbf{R}_{t}^{1 / 2}$ [24], [41] is considered for the proposed QRD-based ED-TAS and EVM-based TAS $(K=3)$ schemes, where $\mathbf{R}_{t}=\left[r_{i j}\right]_{N_{t} \times N_{t}}$ and $\mathbf{R}_{r}=\left[r_{i j}\right]_{N_{r} \times N_{r}}$ are the positive definite Hermitian matrices that specify the transmit and receive correlations, respectively. In Fig. 7, the components of $\mathbf{R}_{t}$ and $\mathbf{R}_{r}$ are calculated as $r_{i j}=r_{j i}^{*}=r^{j-i}$ for $i \leq j$, where $r$ is the correlation coefficient $(0 \leq r \leq 1)$. Here, the simulation parameters are the same as those of Figs. 2 and 4

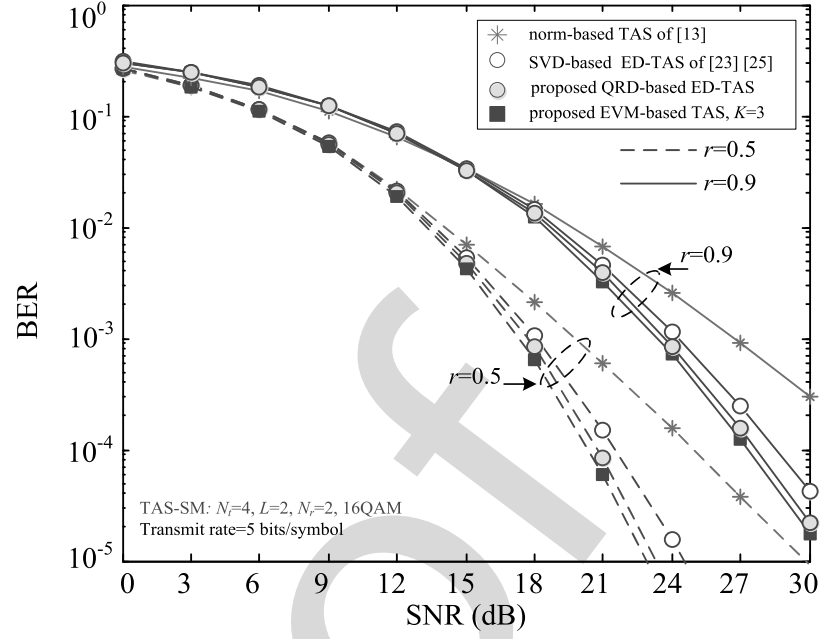

Fig. 7. BER comparison of different TAS algorithms for SM systems in correlated Rayleigh fading channels.

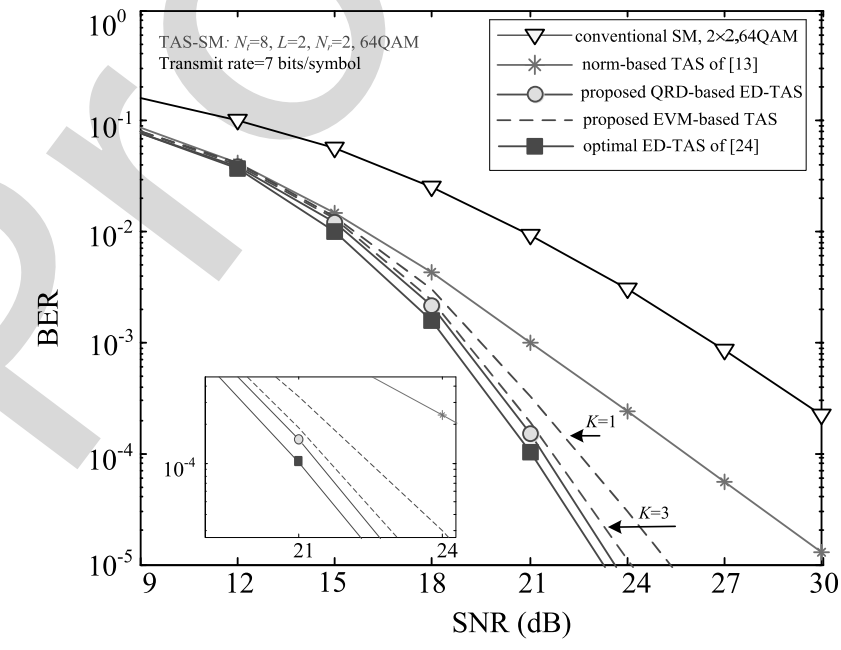

Fig. 8. BER comparison at $m=7$ bits/symbol for the proposed QRD-based ED-TAS and EVM-based ED-TAS with 64-QAM.

for 5 bits/symbol transmissions. We found that the BER curves 660 of the EVM-based TAS schemes and of the optimal ED-TAS 661 are almost overlapped (similar to the results seen in Fig. 4), 662 hence for clarity in Fig. 7 we simply provide the BER curves 663 for the EVM-based TAS schemes only. Compared to the BER 664 curves in Figs. 2 and 4 for the correlation coefficient $r=0$, we 665 observe in Fig. 7 that the BER performance of all schemes is 666 substantially degraded by these correlations. However, the pro- 667 posed schemes remain capable of operating efficiently for the 668 correlated channels.

In Fig. 8, we further compare the proposed QRD-based 670 ED-TAS scheme and the proposed EVM-based TAS schemes 671 for a higher modulation order, where the 64-QAM scheme is 672 employed. Observe in Fig. 8 that the proposed QRD-based 673 ED-TAS scheme outperforms the EVM-based TAS scheme in 674 conjunction with $K=1$ and the corresponding performance 675 gain is seen to be about $1 \mathrm{~dB}$. Similar to the results in Figs. 2676 and 4, the EVM-based TAS associated with $K=3$ provide 677 an improved BER compared to that scheme with $K=1$. At 678 


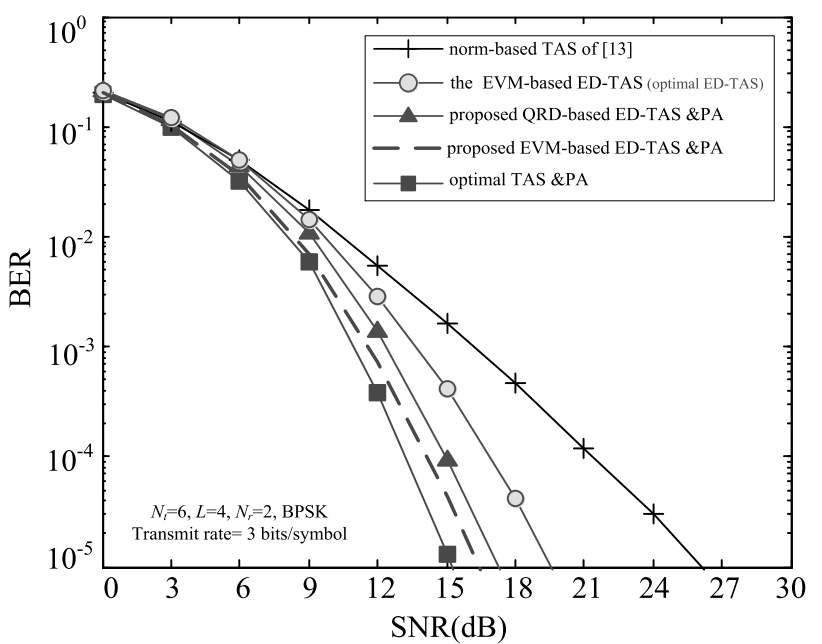

Fig. 9. BER performance comparison of the TAS algorithms and of the proposed TAS \&PA algorithms in SM systems, having the transmit rate of 3 bits/symbol.

$\mathrm{BER}=10^{-5}$, the performance gap between the proposed EVMbased TAS with $K=3$ and the proposed QRD-based ED-TAS becomes negligible.

The main conclusions observed from Figs. 2, 4 and 5-8 are: (1) the proposed EVM-based TAS and QRD-based ED-TAS schemes exhibit different BER advantages for different system setups; (2) the proposed QRD-based ED-TAS is preferred to the QAM-modulated SM schemes, since its complexity is independent of the modulation order; (3) The proposed EVMbased TAS is preferred to the PSK-modulated SM schemes, since it can achieve the performance of optimal ED-TAS at the reduced error vector set. (4) For the QAM-modulated SM schemes, the parameter $K$ of the proposed EVM-based TAS can be flexibly selected for striking a beneficial trade-off between the complexity imposed and the BER attained.

\section{B. BER Comparisons of TAS Algorithms and TAS \&PA Algorithms for SM}

In this subsection, we focus our attention on studying the BER performance of our TAS\&PA algorithms. Here, for the low-complexity TAS\&PA, the proposed QRD-based ED-TAS as well as the EVM-based ED-TAS algorithms are utilized and the corresponding algorithms are termed as the QRD-based ED-TAS \&PA and the EVM-based ED-TAS \&PA, respectively. Note that the EVM-based ED-TAS achieves the same performance as the optimal ED-TAS for the PSK-modulated SM schemes. The BER performances of other TAS algorithms are similar to the results seen in Figs. 2, 4 and 5-8. Hence, for clarity, when only pure TAS is considered, we simply provide the corresponding BER curves of the proposed EVMbased ED-TAS and of the conventional norm-based TAS as benchmarkers.

Fig. 9 compares the BER performance of the proposed TAS\&PA arrangement to that of other SM-based schemes. In Fig. 9, the parameter setup is $N_{t}=6, L=4, N_{r}=2$ and $M=2$. It becomes clear from Fig. 9 that the TAS\&PA algorithms advocated outperform both the EVM-based ED-TAS and

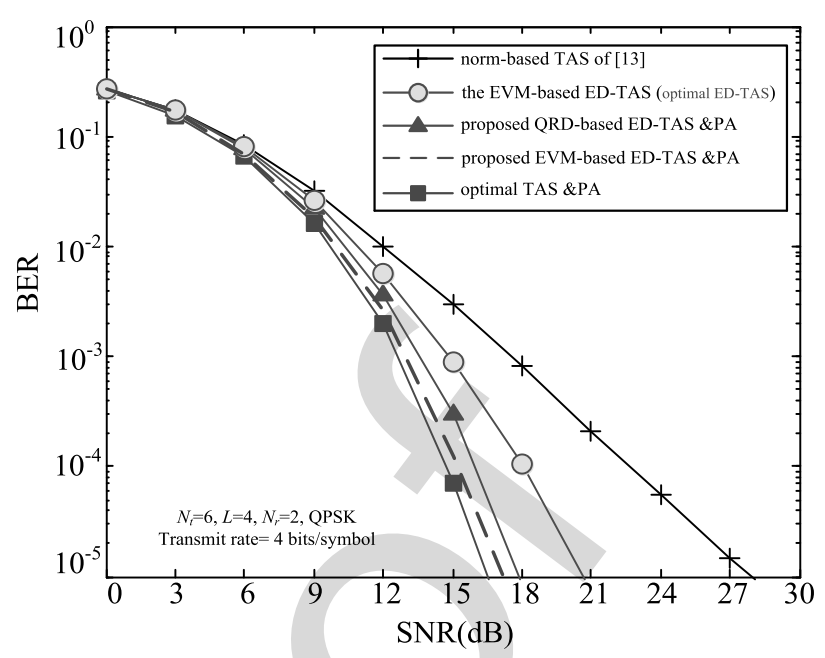

Fig. 10. BER performance comparison of the TAS algorithms and of the proposed TAS \&PA algorithms in SM systems, having the transmit rate of 4 bits/symbol.

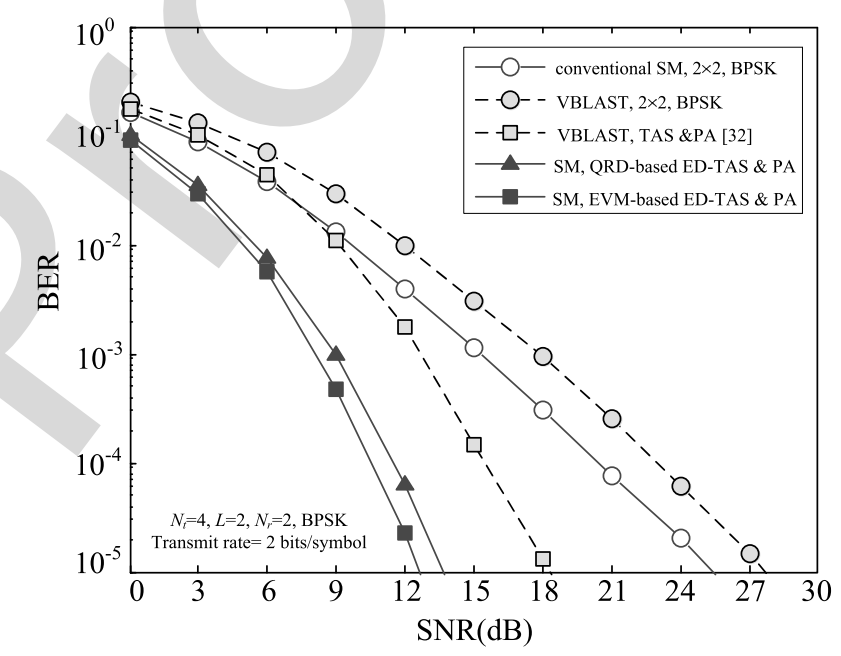

Fig. 11. BER performance comparison of the proposed TAS \&PA algorithms in SM systems and the conventional identical-throughput TAS\&TA algorithm in V-BLAST systems, where the throughput is 2 bits/symbol $\left(N_{t}=4, N_{r}=2\right.$, $L=2)$.

the norm-based ED-TAS. At a BER of $10^{-5}$, the exhaustive- 715 search based optimal TAS\&PA provides $9.5 \mathrm{~dB}$ and $4 \mathrm{~dB}$ SNR 716 gains over the norm-based ED-TAS and over the EVM-based 717 ED-TAS, respectively. Moreover, the low-complexity QRD- 718 based ED-TAS \&PA provides about 4 dB SNR gain over the 719 EVM-based TAS operating without PA.

Fig. 9 also shows that the EVM-based ED-TAS \&PA outper- 721 forms the QRD-based ED-TAS\&PA and is capable of achieving 722 almost the same BER performance as the optimal TAS\&PA. 723 The performance advantages of our schemes are attained as 724 a result of exploiting all the benefits of MIMO channels. The 725 above-mentioned trends of these TAS\&PA algorithms recorded 726 for SM are also visible in Fig. 10, where a SM system using 727 $N_{t}=6, L=4, N_{r}=2$ and QPSK modulation is considered. $\quad 728$

In Fig. 11, the BPSK-modulated V-BLAST scheme and its 729 TAS\&PA-aided counterpart [32] associated with zero-forcing 730 successive interference cancellation (ZF-SIC) are compared to 731 
TABLE II

COMPLEXITY COMPARISON OF DifFERENT TAS-SM ALgORITHMS IN DIVERSE CONFIGURATIONS

\begin{tabular}{|l|r|r|r|}
\hline TAS algorithm & $\begin{array}{r}\text { Configuration 1 } \\
\left(N_{t}=4, N_{r}=2\right. \\
L=2,16 \mathrm{QAM})\end{array}$ & $\begin{array}{r}\text { Configuration 2 } \\
\left(N_{t}=8, N_{r}=4\right. \\
L=4, \mathrm{QPSK})\end{array}$ & $\begin{array}{r}\text { Configuration 3 } \\
\left(N_{t}=8, N_{r}=2\right. \\
L=2,64-\mathrm{QAM})\end{array}$ \\
\hline \hline Exhaustive ED-TAS [13] & 13824 & 8512 & 1032192 \\
\hline Maximum-norm based TAS [21] & 12 & 56 & 24 \\
\hline Conventional QRD-based ED-TAS [24] & 2060 & 6029 & 38253 \\
\hline SVD-based ED-TAS [25] & 102 & 588 & 444 \\
\hline Proposed QRD-based ED-TAS & $\left\{\begin{array}{c}84, K=1 \\
180, K=3\end{array}\right.$ & 596 & 340 \\
\hline Proposed EVM-based ED-TAS & 4626 & 456 & $\left\{\begin{array}{r}360, K=1 \\
808, K=3\end{array}\right.$ \\
\hline Exhaustive TAS\&PA & 853 & 1004 & 256788 \\
\hline Proposed QRD-based ED-TAS\&PA & $\left\{\begin{array}{c}855, K=1 \\
951, K=3\end{array}\right.$ & 1164 & 9511 \\
\hline Proposed EVM-based ED-TAS\&PA & \multicolumn{3}{|c|}{$\begin{array}{r}9531, K=1 \\
9979, K=3\end{array}$} \\
\hline \hline
\end{tabular}

our TAS\&PA based schemes. For maintaining an identicalthroughput, in Fig. 11 we let $N_{t}=4, N_{r}=2, L=2$ and use BPSK for all schemes. Observe in Fig. 11 that our TAS\&PA based SM schemes outperform the TAS\&PA aided V-BLAST schemes by about 5-6 dB SNR at the BER of $10^{-5}$.

\section{Complexity Comparison}

Table I shows the complexity comparison of various TAS algorithms conceived for SM, where the total number of floating point operations is considered. The Appendix provides the details of our computational complexity evaluations for the proposed TAS algorithms list in Table I. The complexity estimation of the existing TAS algorithms can be found in [15], [23] and [24]. Moreover, our complexity analysis is similar to that of [23] and [24].

Explicitly, in Table II, the quantified complexity of different TAS algorithms for some specific configurations are provided. As shown in Table I, the proposed QRD-based ED-TAS has a similar complexity order to that of the low-complexity SVDbased ED-TAS of [23], while exhibiting a lower complexity compared to the conventional QRD-based ED-TAS of [24]. For example, the proposed QRD-based ED-TAS imposes an approximately 168 times and 25 times lower complexity than the exhaustive ED-TAS and the conventional QRD-based EDTAS for configuration 1 . This is due to the fact that it is capable of avoiding the high-complexity QRD operation by directly computing the bound parameters of Eq. (27). Moreover, as shown in Tables I-II and Figs. 4-8, the EVM-based ED-TAS advocated is capable of striking a flexible BER vs complexity trade-off by employing the parameter $K$ for diverse $M$-QAM schemes. Furthermore, the proposed low-complexity TAS\&PA schemes impose a lower complexity than the exhaustive-search based TAS\&PA and only impose a slightly increased complexity compared to the proposed EVM-based TAS and QRD-based TAS schemes. By considering the BER vs complexity results of Tables I-II and Figs. 9-11, the proposed low-complexity TAS\&PA is seen to provide an improved BER performance at a modest complexity cost.

\section{CONCLUSiOnS}

In this paper, we have investigated TAS algorithms conceived for SM systems. Firstly, a pair of low-complexity
ED-TAS algorithms, namely the QRD-based ED-TAS and the 772 EVM-based ED-TAS, were proposed. The theoretical analysis 773 and simulation results indicated that the QRD-based ED-TAS 774 exhibits a better BER performance compared with the conven- 775 tional SVD-based ED-TAS, while the EVM-based ED-TAS is 776 capable of striking a flexible BER vs complexity trade-off. To 777 further improve the attainable performance, the proposed TAS 778 algorithms were amalgamated with PA. A pair of beneficial 779 joint TAS-PA algorithms were proposed and our simulation 780 results demonstrated that they outperform both the pure TAS 781 algorithms and the TAS\&PA algorithm designed for spatial 782 multiplexing systems.

\section{APPENDIX}

Computational complexity of the proposed TAS algorithms 785 designed for SM systems.

\section{A. The Proposed QRD-Based ED-TAS}

As detailed in Section IV-A, the calculation of the QRD- 788 based bound of Eq. (27) only depends on the elements of 789 the matrix $\mathbf{H}^{H} \mathbf{H}$, which incurs a complexity in the order 790 of $\operatorname{comp}\left(\mathbf{H}^{H} \mathbf{H}\right)=2 N_{t}^{2} N_{r}-N_{t}^{2}$. Then, we can calculate the 791 values of $\tilde{R}_{k, k}\left(\boldsymbol{\Pi}_{m}\right),(m=1,2, k=1,2)$ in Eqs. (30)-(33) 792 by reusing these elements for the different TAS candi- 793 dates $\mathbf{H}_{u}$. Specifically, the calculation of $\sqrt{\left\|\mathbf{h}_{u}(j)\right\|_{F}^{2}}, j=794$ $1, \cdots, N_{t}$ for estimating $\tilde{R}_{1,1}\left(\boldsymbol{\Pi}_{m}\right), m=1,2$ in Eqs. (30) and 795 (32) requires $N_{t}$ flops. Moreover, to calculate the values of 796 $\tilde{R}_{2,2}\left(\boldsymbol{\Pi}_{m}\right), m=1,2$ in Eqs. (31) and (33), we have to con- 797 sider $\left(\begin{array}{c}N_{t} \\ 2\end{array}\right)$ possible combinations $(i, j)$ for computing the value 798 of $\sqrt{\frac{\left\|\mathbf{h}_{u}(i)\right\|_{F}^{2}+\left\|\mathbf{h}_{u}(j)\right\|_{F}^{2}-2 \mathcal{R}\left\{\mathbf{h}_{u}(i)^{H} \mathbf{h}_{u}(j)\right\}}{\left\|\mathbf{h}_{u}(j)\right\|_{F}^{2}}}$. For each combination, 799 the complexity imposed is 5 flops. Hence, the complexity 800 of computing $\tilde{R}_{2,2}\left(\boldsymbol{\Pi}_{m}\right), m=1,2$ is $5\left(\begin{array}{c}N_{t} \\ 2\end{array}\right)$ flops. The overall 801 complexity of the proposed QRD-based ED-TAS is

$$
\begin{aligned}
C_{\mathrm{PQRD}} & =2 N_{t}^{2} N_{r}-N_{t}^{2}+N_{t}+5\left(\begin{array}{c}
N_{t} \\
2
\end{array}\right) \\
& =2 N_{t}^{2} N_{r}+\frac{3}{2} N_{t}\left(N_{t}-1\right) .
\end{aligned}
$$

Note that based on Eq. (28), $d_{\min }^{\text {Modulus }}, d_{\min }^{\mathrm{APM}}$ and $d_{\min }^{\text {all }}$ are 803 constants for a specific APM scheme and the calculation of 804 $d_{\text {min }}^{\text {signal }}$ and $d_{\text {min }}^{\text {spatial }}$ can also exploit the common elements, such 805 as $\left\|\mathbf{h}_{u}(i)\right\|_{F}^{2}+\left\|\mathbf{h}_{u}(j)\right\|_{F}^{2}-2 \mathcal{R}\left\{\mathbf{h}_{u}(i)^{H} \mathbf{h}_{u}(j)\right\},\left\|\mathbf{h}_{u}(i)\right\|_{F}^{2}$, in the 806 
807 calculation of the bound of $d_{\min }^{\text {joint }}$, as shown in Eqs. (15) and 808

(16). Hence, the complexity imposed can be deemed negligible.

\section{B. The Proposed EVM-Based ED-TAS}

Similar to the proposed QRD-based ED-TAS, the computational complexity of EVM-based ED-TAS is also dominated by computing $d_{\min }^{\text {joint }}$. Specifically, we also first have to evaluate the elements $\left\|\mathbf{h}_{u}(i)\right\|_{F}^{2},\left\|\mathbf{h}_{u}(j)\right\|_{F}^{2}$ and $\mathbf{h}_{u}(i)^{H} \mathbf{h}_{u}(j)$, which incurs a complexity of $2 N_{t}^{2} N_{r}-N_{t}^{2}$ flops. Then, for $M$-PSK, the simplified version of $d_{\mathrm{min}}^{\text {joint }}$ is given in Eq. (40), which has to consider $\left(\begin{array}{c}N_{t} \\ 2\end{array}\right)$ legitimate TA combination $(i, j)$. For each combination $(i, j)$, the computation of the term $m_{M-\mathrm{PSK}}\left(\mathbf{H}_{u}\right)$ of Eq. (39) has to consider $\left(\frac{M}{4}+1\right)$ possible $\theta_{n}$ values. For each $\theta_{n}$, the complexity of evaluating $\left|\mathcal{R}\left\{\mathbf{h}_{u}(i){ }^{H} \mathbf{h}_{u}(j)\right\} \cos \theta_{n}-\mathcal{J}\left\{\mathbf{h}_{u}(i){ }^{H} \mathbf{h}_{u}(j)\right\} \sin \theta_{n}\right|$ is 4 flops. Moreover, for a specific $m_{M-\operatorname{PSK}}\left(\mathbf{H}_{u}\right)$ and a fixed combination $(i, j)$, the computation of $\left\|\mathbf{h}_{u}(i)\right\|_{F}^{2}+\left\|\mathbf{h}_{u}(j)\right\|_{F}^{2}-$ $2 m_{M-\mathrm{PSK}}\left(\mathbf{H}_{u}\right)$ in Eq. (40) requires 3 flops. Hence, the overall complexity of the $M$-PSK modulated EVM-based ED-TAS is

$$
\begin{aligned}
C_{\mathrm{EVM}} & =2 N_{t}^{2} N_{r}-N_{t}^{2}+\left(\begin{array}{c}
N_{t} \\
2
\end{array}\right)\left\{4\left(\frac{M}{4}+1\right)+3\right\} \\
& =2 N_{t}^{2} N_{r}-N_{t}^{2}+\frac{1}{2} N_{t}\left(N_{t}-1\right)(M+7) .
\end{aligned}
$$

For the $M$-QAM scheme, this complexity depends on the parameter $K$. Specifically, the simplified versions of $d_{\min }^{\text {joint }}$ are different for different values of $K$. In general, for a given $K$, we first characterize all possible combinations of $\left|s_{a}\right|^{2}$ and $\left|s_{b}\right|^{2}$ by using the method of Section IV-B. Let us assume that the number of these combinations is $G$. For each combination, we can simplify Eq. (37) similar to the process of Eqs. (43)-(44), which corresponds to $G$ simplified equations and each requires 15 flops, as shown in Eq. (37). Since $\left(\begin{array}{c}N_{t} \\ 2\end{array}\right)$ legitimate TA combinations $(i, j)$ should be considered in Eq. (37), we arrive at a complexity of $15 G\left(\begin{array}{c}N_{t} \\ 2\end{array}\right)$ for all possible combinations. Overall, the complexity of the EVM-based TAS for $M$-QAM modulated $\mathrm{SM}$ is

$$
C_{\mathrm{EVM}}=2 N_{t}^{2} N_{r}-N_{t}^{2}+15 G\left(\begin{array}{c}
N_{t} \\
2
\end{array}\right) .
$$

Note that the complexity of Eq. (49) is an approximate result, which can be further refined based on the specific simplified version of $d_{\min }^{\text {joint }}$. For example, based on Eqs. (41) and (43) derived for $K=1$ and $K=3$, similar to the complexity analysis of $M$-PSK, the computational complexity orders of the EVM-based TAS for $K=1$ and $K=3$ are

$$
C_{\mathrm{EVM}-\mathrm{TAS}}=2 N_{t}^{2} N_{r}-N_{t}^{2}+6\left(\begin{array}{c}
N_{t} \\
2
\end{array}\right),
$$

and

$$
C_{\mathrm{EVM}-\mathrm{TAS}}=2 N_{t}^{2} N_{r}-N_{t}^{2}+22\left(\begin{array}{c}
N_{t} \\
2
\end{array}\right)
$$

\section{The Proposed PA \& TAS}

The exhaustive-search based TAS\&PA algorithm has to calculate all legitimate PA matrix candidates. According to Section
$\mathrm{V}$, there are $N_{U}=\left(\begin{array}{c}N_{t} \\ L\end{array}\right)$ legitimate PA matrix candidates $\mathbf{P}_{u}(u=848$ $\left.1, \cdots, N_{U}\right)$, which can be obtained by using the method pro- 849 posed in [29]. The complexity of computing each PA matrix is 850 $C_{\mathrm{PA}}$ (Eq. (22) in [29]) flops. Hence, the associated complexity 851 of the exhaustive-search based TAS\&PA algorithm is $N_{U} C_{\mathrm{PA}} 852$ flops. By contrast, the low-complexity TAS\&PA algorithm first 853 selects the optimal TA subset and then calculates the PA matrix 854 for the selected set. Hence, the associated complexity order of 855 the low-complexity TAS\&PA algorithm is $C_{\mathrm{TAS}}+C_{\mathrm{PA}}$ flops, 856 where $C_{\text {TAS }}$ is the complexity of the TAS algorithm employed, 857 i. e. $C_{\mathrm{EVM}}$ or $C_{\mathrm{PQRD}}$.

\section{REFERENCES}

[1] R. Y. Mesleh, H. Haas, S. Sinanovic, C. W. Ahn, and S. Yun, "Spatial 860 modulation," IEEE Trans. Veh. Technol., vol. 57, no. 4, pp. 2228-2241, 861 Jul. 2008.

[2] M. Di Renzo, H. Haas, A. Ghrayeb, S. Sugiura, and L. Hanzo, "Spatial 863 modulation for generalized MIMO: Challenges, opportunities and imple- 864 mentation," Proc. IEEE, vol. 102, no. 1, pp. 56-103, Jan. 2014.

[3] S. Sugiura, S. Chen, and L. Hanzo, "A universal space-time architec- 866 ture for multiple-antenna aided systems," IEEE Commun. Surveys Tuts., 867 vol. 14, no. 2, pp. 401-420, May 2012.

[4] M. Di Renzo, H. Haas, and P. M. Grant, "Spatial modulation for multiple- 869 antenna wireless systems: A survey," IEEE Commun. Mag., vol. 49, 870 no. 12, pp. 182-191, Dec. 2011.

[5] A. Stavridis, S. Sinanovic, M. Di Renzo, and H. Haas, "Energy evaluation 872 of spatial modulation at a multi-antenna base station," in Proc. IEEE Veh. 873 Technol. Conf., Barcelona, Spain, Sep. 2013, pp. 1-5.

[6] M. Di Renzo and H. Haas, "Bit error probability of SM-MIMO over 875 generalized fading channels," IEEE Trans. Veh. Technol., vol. 61, no. 3, 876 $\begin{array}{ll}\text { pp. 1124-1144, Mar. 2012. } & 877\end{array}$

[7] P. Yang, M. Di Renzo, Y. Xiao, S. Li, and L. Hanzo, "Design guidelines 878 for spatial modulation," IEEE Commun. Surveys Tuts., vol. 17, no. 1, 879 pp. 6-26, Mar. 2015.

[8] S. Sugiura and L. Hanzo, "On the joint optimization of dispersion matri- 881 ces and constellations for near-capacity irregular precoded space-time 882 shift keying," IEEE Wireless Commun., vol. 12, no. 1, pp. 380-387, Jan. 883 2013.

[9] P. Yang, Y. Xiao, Y. Yi, and S. Li, "Adaptive spatial modulation for wire- 885 less MIMO transmission systems," IEEE Commun. Lett., vol. 15, no. 6, 886 pp. 602-604, Jun. 2011.

[10] P. Yang, Y. Xiao, L. Li, Q. Tang, Y. Yu, and S. Li, "Link adaptation for 888 spatial modulation with limited feedback," IEEE Trans. Veh. Technol., 889 vol. 61 , no. 8, pp. $3808-3813$, Oct. 2012.

[11] M. Di Renzo and H. Haas, "Improving the performance of space shift 891 keying (SSK) modulation via opportunistic power allocation," IEEE 892 Commun. Lett., vol. 14, no. 6, pp. 500-502, Jun. 2010.

[12] S. Sanayei and A. Nosratinia, "Antenna selection in MIMO systems," 894 IEEE Commun. Mag., vol. 42, no. 10, pp. 68-73, Oct. 2004.

[13] W. H. Chung, C. Y. Hung, Q. Zhang, and M. Gao, "Multi-antenna selec- 896 tion using space shift keying in MIMO systems," in Proc. IEEE Veh. 897 Technol. Conf., May 2012, pp. 1-5.

[14] B. Kumbhani and R. S. Kshetrimayum, "Outage probability analysis 899 of spatial modulation systems with antenna selection," Electron. Lett., 900 vol. 50, no. 2, pp. 125-126, 2014.

[15] S. Sugiura, S. Chen, and L. Hanzo, "Coherent and differential space- 902 time shift keying: A dispersion matrix approach," IEEE Trans. Commun., 903 vol. 58, no. 11, pp. 3219-3230, Nov. 2010.

[16] J Wang et al., "Closed-loop spatial modulation with antenna selection," 905 in Proc. IEEE 11th Int. Conf. Signal Process., Oct. 2012, pp. 1291-1294. 906

[17] Z. Zhou, N. Ge, and X. Lin, "Reduced-complexity antenna selection 907 schemes in spatial modulation," IEEE Commun. Lett., vol. 18, no. 1, 908 pp. 14-17, Jun. 2014.

[18] M. Maleki, H. R. Bahrami, and A. Alizadeh, "Adaptive antenna sub- 910 set selection and constellation breakdown for spatial modulation," IEEE 911 Commun. Lett., vol. 18, no. 9, pp. 1649-1652, Sep. 2014.

[19] X. Wu, M. D. Renzo, and H. Haas, "Direct transmit antenna selection 913 for transmit optimized spatial modulation," in Proc. IEEE Veh. Technol. 914 Conf. (VTC'13-Fall), Las Vegas, NV, USA, Sep. 2013, pp. 1-5. 915

[20] X. Wu, M. Di Renzo, and M. Haas, "Adaptive selection of antennas 916 for optimum transmission in spatial modulation," IEEE Trans. Wireless 917 Commun., vol. 14, no. 7, pp. 3630-3641, Jul. 2015. 
[21] R. Rajashekar, K. V. S. Hari, and L. Hanzo, "Antenna selection in spatial modulation systems," IEEE Commun. Lett., vol. 17, no. 3, pp. 521-524, Mar. 2013.

[22] N. Pillay and H. Xu, "Comments on "Antenna selection in spatial modulation systems"," IEEE Commun. Lett., vol. 17, no. 9, pp. 1681-1683, Sep. 2013.

[23] K. Ntontin, M. Di Renzo, A. Perez-Neira, and C. Verikoukis, "A lowcomplexity method for antenna selection in spatial modulation systems," IEEE Commun. Lett., vol. 17, no. 12, pp. 2312-2315, Aug. 2013.

[24] N. Wang, W. Liu, H. Men, M. Jin, and H. Xu. "Further complexity reduction using rotational symmetry for EDAS in spatial modulation," IEEE Commun. Lett., vol. 18, no. 10, pp. 1835-1838, Oct. 2014.

[25] J. Zheng and J. Chen, "Further complexity reduction for antenna selection in spatial modulation systems," IEEE Commun. Lett., vol. 19, no. 6 , pp. 937-940, Jun. 2015.

[26] R. Rajashekar, K. V. S. Hari, and L. Hanzo, "Quantifying the transmit diversity order of Euclidean distance based antenna selection in spatial modulation," IEEE Commun. Lett., vol. 22, no. 9, pp. 1434-1437, Sep. 2015.

[27] N. Pillay and X. Hongjun, "Low-complexity transmit antenna selection schemes for spatial modulation," IET Commun., vol. 9, no. 2, pp. 239248, Jan. 2015.

[28] P. Yang, Y. Xiao, B. Zhang, S. Li, M. El-Hajjar, and L. Hanzo, "Power allocation aided spatial modulation for limited-feedback MIMO systems," IEEE Trans. Veh. Technol., vol. 64, no. 5, pp. 2198-2204, May 2015.

[29] P. Yang, Y. Xiao, S. Li, and L. Hanzo, "A low-complexity power allocation algorithm for multiple-input multiple-output spatial modulation systems," IEEE Trans. Veh. Technol., vol. 65, no. 3, pp. 1819-1825, Mar 2016.

[30] M. Di Renzo and H. Haas, "Improving the performance of space shift keying (SSK) modulation via opportunistic power allocation," IEEE Commun. Lett., vol. 14, no. 6, pp. 500-502, Jun. 2010.

[31] Y. Xiao, Q. Tang, L. Gong, P. Yang, and Z. Yang, "Power scaling for spatial modulation with limited feedback," Int. J. Antennas Propag., vol. 20135 p., 2013

[32] Z. Shi and H. Leib, "Transmit antenna selection V-BLAST systems with power allocation," IEEE Trans. Veh. Technol., vol. 57, no. 4, pp. 2293 2304, Jul. 2008

[33] R. Rajashekar, K. V. S. Hari, and L. Hanzo, "Reduced-complexity ML detection and capacity-optimized training for spatial modulation," IEEE Trans. Commun., vol. 62, no. 1, pp. 112-125, Jan. 2014.

[34] S. Sugiura, C. Xu, S. X. Ng, and L. Hanzo, "Reduced complexity coherent versus non-coherent QAM-aided space time shift keying," IEEE Trans. Commun., vol. 59, no. 11, pp. 3090-3101, Nov. 2011.

[35] A. Younis, S. Sinanovic, M. Di Renzo, R. Y. Mesleh, and H. Haas, "Generalised sphere decoding for spatial modulation," IEEE Trans. Commun., vol. 61, no. 7, pp. 2805-2815, Jul. 2013.

[36] A. Goldsmith, Wireless Communications. Cambridge, U.K.: Cambridge Univ. Press, 2005.

[37] G. Golub and C. F. van Loan, Matrix Computations, 3 rd ed. Baltimore, MD, USA: The Johns Hopkins Univ. Press, 1996.

[38] J. K. Zhang, A. Kavcic, and K. M. Wong, "Equal-diagonal QR decomposition and its application to precoder design for successive-cancellation detection," IEEE Trans. Inf. Theory, vol. 51, no. 1, pp. 154-172, Jan. 2005.

[39] J. Lee, S. Y. Jung, and D. Park, "Simplified maximum-likelihood precoder selection for spatial multiplexing systems," IEEE Trans. Veh. Technol., vol. 59, no. 9, pp. 4628-4634, Nov. 2010.

[40] Z. Chen, J. Yuan, and B. Vucetic, "Analysis of transmit antenna selection/maximal-ratio combining in Rayleigh fading channels," IEEE Trans. Veh. Techol., vol. 54, no. 4, pp. 1312-1321, Jul. 2005.

[41] V. Raghavan, J. J. Choi, and D. J. Love, "Design guidelines for limited feedback in the spatially correlated broadcast channel," IEEE Trans. Commun, vol. 63, no. 7, pp. 2524-2540, Jul. 2015.

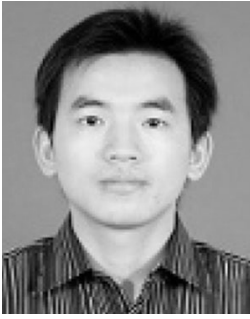

Ping Yang (SM'xx) received the Ph.D. degree from the University of Electronic Science and Technology of China (UESTC), Chengdu, China, in 2013. From September 2012 to September 2013, he was a Visiting Student at the School of Electronics and Computer Science, University of Southampton, Southampton, U.K. Since May 2014, he has been a Research Fellow in EEE of NTU, Singapore. Also, he is an Assistant Professor with UESTC. His research interests include MIMO/OFDM, machine learning, life science, and communication signal processing.

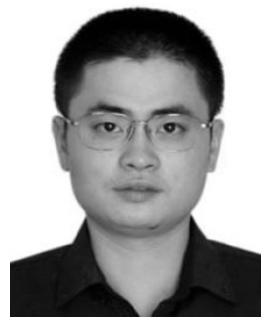

Yue Xiao (M'xx) received the Ph.D. degree in 995 communication and information systems from the 996 University of Electronic Science and Technology of 997 China, Chengdu, China, in 2007. He is now a Full 998 Professor with the University of Electronic Science 999 and Technology of China. He has authored more than 1000 30 international journals and been involved in several 1001 projects in Chinese Beyond 3G Communication R\&D 1002 Program. His research interests include wireless and 1003 mobile communications.

1004

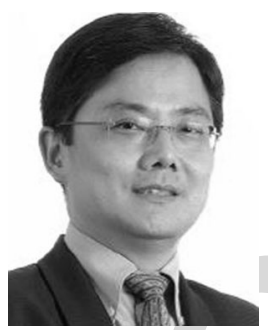

Yong Liang Guan (M'xx) received the Ph.D. degree 1005 from the Imperial College of Science, Technology, 1006 and Medicine, University of London, London, U.K., 1007 in 1997, and the B.Eng. degree (with first class 1008 Hons.) from the National University of Singapore, 1009 Singapore, in 1991. He is now an Associate 1010 Professor with the School of Electrical and Electronic 1011 Engineering, Nanyang Technological University, 1012 Singapore. His research interests include modulation, 1013 coding and signal processing for communication, 1014 information security and storage systems.

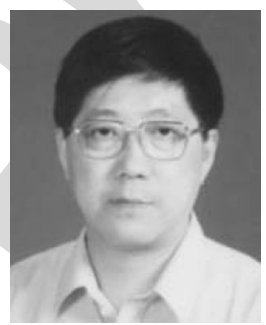

Shaoqian Li (F'16) received the B.S.E. degree in 1016 communication technology from Northwest Institute 1017 of Telecommunication (Xidian University), Xi'an, 1018 China, in 1982, and the M.S.E. degree in communica- 1019 tion system from the University of Electronic Science 1020 and Technology of China (UESTC), Chengdu, China, 1021 in 1984. He is a Professor, Ph.D. Supervisor, 1022 and the Director of the National Key Laboratory 1023 of Communication, UESTC, and member of the 1024 National High Technology R\&D Program (863 1025 Program) Communications Group. His research inter- 1026 ests includes wireless communication theory, anti-interference technology for 1027 wireless communications, spread-spectrum and frequency-hopping technology, 1028 and mobile and personal communications.

1029

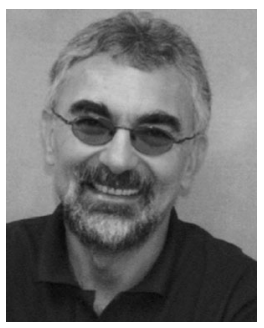

Lajos Hanzo (F'xx) received the degree in elec- 1030 tronics, in 1976, the doctorate degree, in 1983, 1031 and the D.Sc. degree. During his 37-year career in 1032 telecommunications, he has held various research 1033 and academic posts in Hungary, Germany, and the 1034 U.K. Since 1986, he has been with the School of 1035 Electronics and Computer Science, University of 1036 Southampton, Southampton, U.K., where he holds 1037 the chair in telecommunications. He has successfully 1038 supervised over $80 \mathrm{Ph} . \mathrm{D}$. students, coauthored 201039 John Wiley/IEEE Press books on mobile radio com- 1040 munications totalling in excess of 10000 pages, published 1400+ research 1041 entries at IEEE Xplore, acted both as TPC and the General Chair of the IEEE 1042 conferences, presented keynote lectures and has been awarded a number of 1043 distinctions. Currently, he is directing a 100-strong academic research team, 1044 working on a range of research projects in the field of wireless multimedia 1045 communications sponsored by industry, the Engineering and Physical Sciences 1046 Research Council (EPSRC) U.K., the European Research Council's Advanced 1047 Fellow Grant and the Royal Society's Wolfson Research Merit Award. He is an 1048 enthusiastic supporter of industrial and academic liaison and offers a range of 1049 industrial courses. He is also a Governor of the IEEE VTS. From 2008 to 2012, 1050 he was the Editor-in-Chief of the IEEE Press and a Chaired Professor also at 1051 Tsinghua University, Beijing, China. His research is funded by the European 1052 Research Council's Senior Research Fellow Grant. He has more than 190001053 citations. In 2009, he was received the honorary doctorate "Doctor Honoris 1054 Causa" by the Technical University of Budapest, Budapest, Hungary. He is the 1055 fellow of the REng, IET, and EURASIP. 


\section{QUERIES}

Q1: Please be advised that per instructions from the Communications Society this proof was formatted in Times Roman font and therefore some of the fonts will appear different from the fonts in your originally submitted manuscript. For instance, the math calligraphy font may appear different due to usage of the usepackage[mathcal]euscript. We are no longer permitted to use Computer Modern fonts.

Q2: Note that if you require corrections/changes to tables or figures, you must supply the revised files, as these items are not edited for you.

Q3: Please provide IEEE membership details of authors "Ping Yang, Yue Xiao, Yong Liang Guan, and Lajos Hanzo.” 